\title{
Baseline Assessments for Fish, Macroinvertebrates, and Herpetofauna in the Headwaters of Otter and Hanging Woman Creeks within the Tongue Powder CBNG Area
}

Prepared for:

\author{
Bureau of Land Management - Miles City Field Office and \\ the Interagency BLM Aquatic Task Group \\ Prepared by: \\ David Stagliano
}

Montana Natural Heritage Program a cooperative program of the

Montana State Library and the University of Montana

June 2011

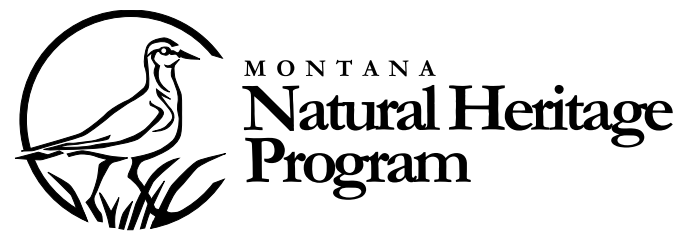





\section{Baseline Assessments for Fish, Macroinvertebrates, and Herpetofauna in the Headwaters of Otter and Hanging Woman Creeks within the Tongue Powder CBNG Area}

Prepared for:

Bureau of Land Management - Miles City Field Office and the Interagency BLM Aquatic Task Group

Agreement Number:

L08AC13222

Prepared by:

David Stagliano

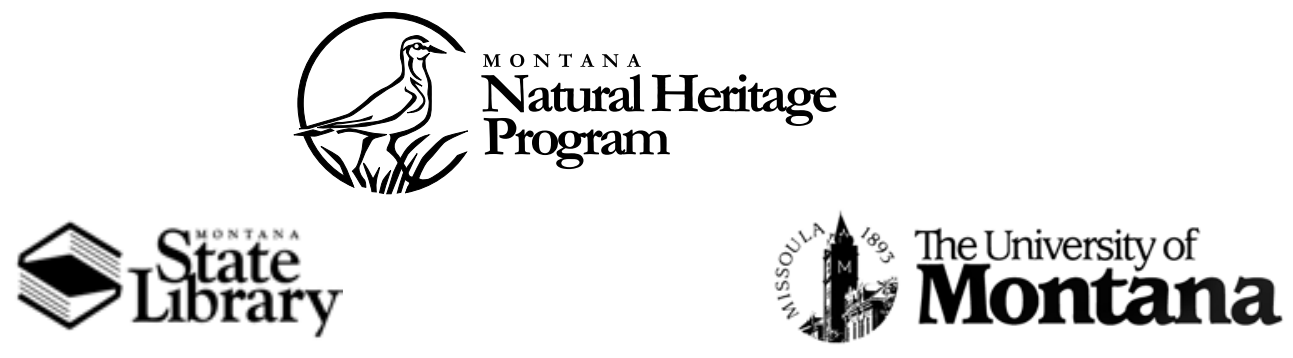

(C) 2011 Montana Natural Heritage Program

P.O. Box $201800 \bullet 1515$ East Sixth Avenue • Helena, MT 59620-1800 • 406-444-5354 
This document should be cited as follows:

Stagliano, David. 2011. Baseline Assessments for Fish, Macroinvertebrates, and Herpetofauna in the Headwaters of Otter and Hanging Woman Creeks within the Tongue Powder CBNG Area. Report to the United States Bureau of Land Management and the Aquatic Task Force. Montana Natural Heritage Program, Helena, Montana. 17 pp. plus appendices. 


\section{Executive Summary}

This report summarizes the first year of activities on the Aquatic Baseline Assessment for Fish, Macroinvertebrates, and Herpetofauna in the Headwaters of Otter and Hanging Woman Creeks. Project goals include: 1) to survey and collect baseline information about the aquatic communities occurring in the Northwestern Great Plains Intermittent Stream ecological systems within unsurveyed portions of the headwaters of these two streams prior to CBNG development; and 2) to assess aquatic community integrity by identifying and interpreting key community indicators found at the sites using standardized protocols and biotic thresholds, and to compare these against reference condition standards at the watershed level and local reach scale.

Habitat assessments, water quality measurements, herpotofauna, macroinvertebrate and/or fish surveys were performed at 20 predetermined lotic (stream channel) reaches (per conversation with J. Chaffin). These included sites on upper Otter and Hanging Woman Creeks, Trail and Bear Creeks and any tributaries containing suitable aquatic habitats. In total, we evaluated 87 stream reaches, of which 27 were dry reaches or ephemeral tributaries. Sixty stream reaches were seined or dip-netted for fish, of these, only 12 sites contained fish while 37 sites contained amphibians. Biological community integrity was calculated at 12 sites using Fish Integrated Biotic Indices (IBI's) and Observed/Expected Models (O/E), 20 sites were assessed with macroinvertebrate multi-metrics (MT MMI). The Northwestern Great Plains Intermittent Prairie Stream ecological system, which dominates this region, may not always contain fish, but is an important breeding and rearing areas for many species of amphibians and reptiles.

Habitat Evaluations. Of 87 sampling reaches evaluated within the study area, we found 32 in Proper Functioning Condition (PFC) with a stable trend; 49 were Functional at Risk (FAR) and 6 were ranked Nonfunctional (NF). Highest site integrity scores using both BLM Buglab stream (24 max. score) and PFC habitat assessment methods were recorded at the Hanging Woman Creek (HWC) sites ATG_143, a sedge meadow channel and ATG_137, a run-pool stream channel just upstream of the Montana border into Wyoming. Other fairly intact sites were near the lower 76 Creek and Trail Creek confluence, Hanging Woman Creek off Quietus Road (ATG_200, cover photo) and SF Taylor Creek. Sites with lower habitat scores were predominately degraded structurally by cattle usage and had associated high CPI values (upper Seventysix Creek and Trial Creek \#183). The water quality parameters (conductivity \& turbidity) recorded at both Corral and upper Otter Creek sites were above the threshold for impairment levels (DEQ 2006), and the Otter Creek site had visible signs of petroleum seepage from ground water.

Macroinvertebrate Communities. Overall, 110 unique macroinvertebrate taxa were reported from the 20 invertebrate assessment sites. No macroinvertebrate species of concern (SOC) were collected. Average macroinvertebrate taxa richness per site was 24.8 and the highest taxa richness reported at two Otter Creek sites was 36 taxa. Using the Montana DEQ macroinvertebrate multimetric index (MMI), 14 of the 20 sites were ranked nonimpaired (good to excellent biological integrity), five were marginally unimpaired and one slightly impaired. Hanging Woman Creek sites \#143 \& \#196 had reference condition macroinvertebrate scores for a Great Plains Prairie Stream with DEQ MMI scores of 69.4 and 76.6, respectively. Most stream sites that contained flowing connected water scored higher with the MMI than sites with exclusively interrupted pool areas. Overall, sites evaluated in the Hanging Woman Creek basin received higher macroinvertebrate MMI scores than those in the Otter Creek basin.

Fish Communities. Overall, seven fish species (four native/three introduced) were identified from 1,219 individuals collected from 12 lotic ATG sites containing fish (Table 2). All fish sites also reported amphibians present. Fathead minnows had the highest site occupancy rate $92 \%$ (11 of 12 sites) and abundance, proportionally contributing $71 \%$ of all individuals collected. Brassy minnows were the next most collected species at seven sites. Fish data collected in previous years from three sites 
within the study area were evaluated for biological integrity changes over time. The most diverse site was the Otter Creek site at the old CCC cabin with five species (four native) and the most intact sites were Otter Creek at Taylor Creek and Fifteenmile Roads, each with four native species. Using Montana's Prairie Fish IBI, four of the 12 fish sites were ranked non-impaired (good biological integrity), eight were slightly impaired (moderate integrity) and two were moderately impaired (poor biotic integrity). No sites where fish were collected were ranked severely impaired by the Fish IBI, although some sites with no fish had habitat potential for fish to be present, but may have been limited by connectivity to colonization sources.

Amphibian and Reptile Incidentals. Thirteen herpetofauna species were collected in dipnets while seining and incidentally recorded in conjunction with the habitat and macroinvertebrate surveys. Woodhouse's Toad had the highest site occupancy, occurring at 37 of 60 sites, followed by the Tiger Salamander and Boreal Chorus Frog recorded at 27 and 26 sites, respectively. We reported six amphibian species. In order of site occupancy, they were: Woodhouse's Toad (Bufo woodhousii); Tiger Salamander (Ambystoma tigrinum); Boreal Chorus Frog (Psuedacris maculate); Northern Leopard Frog (Rana pipiens); Plains Spadefoot Toad (Spea bombifrons) - SOC; and Great Plains Toad (Bufo cognatus). We also found seven reptile species (again in order of site occurrence): Painted Turtle (Chrysemys picta); Terrestrial Garter Snake (Thamnophis elegans); Western Rattlesnake (Crotalus viridis); Plains Garter Snake(Thamnophis radix); Western Hognose Snake (Heterodon nasicus) - SOC; and Snapping Turtle (Chelydra serpentina) - SOC. 


\section{ACKNOWLedgements}

We would like to thank Jake Chaffin of the Bureau of Land Management - Miles City Field Office and the Interagency BLM Aquatic Task Group, headed by Bill Osthiemer of the BLM Wyoming for funding this project under agreement \# L08AC13222.

Report review and editing was provided by Bryce Maxell and Linda Vance. Fieldwork assistance was provided by Brent Cascadian, map production by Meghan Burns and report formatting by Coburn Currier (MTNHP).

All photos in the report were taken by MTNHP personnel, unless otherwise noted. 


\section{Table of Contents}

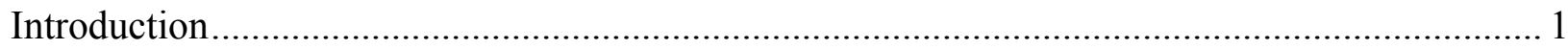

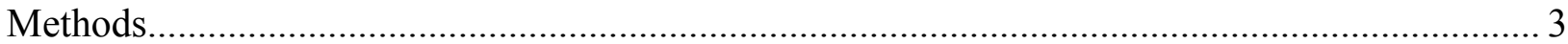

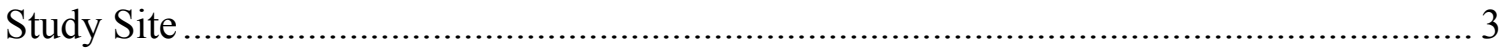

Riparian Assessments............................................................................................... 3

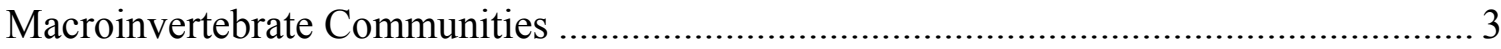

Fish and Amphibian Surveys ………………………............................................. 4

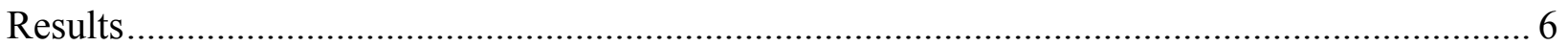

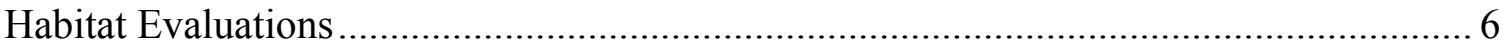

Macroinvertebrate Communities .......................................................................... 9

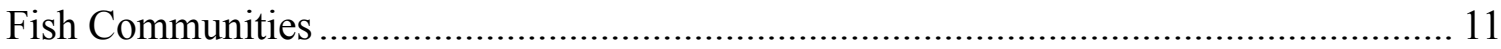

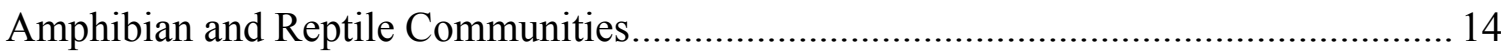

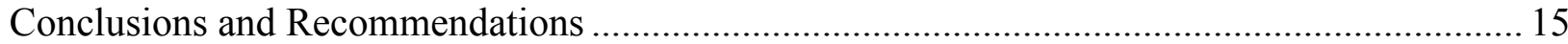

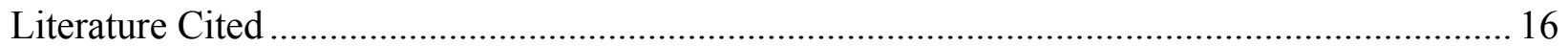

Appendix A: All sites visited and site information collected during the 2010 ATG Ripairian Assessments

Appendix B: Macroinvertebate taxa, abundance and metrics for the 20 collection sites

Appendix C: Raw fish data and IBI metric calculations collected from Upper Hanging Woman and Otter Creek Sites

\section{List OF Figures}

Figure 1. 2010 study area location in the Tongue and Powder River Basins.............................. 2

Figure 2. Stream Aquatic Assessment 2010 study area locations ............................................... 7

Figure 3. Riparian functional assessment rankings for 2010 reach locations .............................. 8

Figure 4. Photo of a structurally cattle-damaged, non-functional (NF) stream reach ................ 9

Figure 5. Fish and amphibian presence locations recorded during 2010 surveys....................... 10

Figure 6. Habitat and macroinvertebrate MMI score relationships ....................................... 12

Figure 7. Photo of brassy minnows in spawning colors and white sucker .............................. 13

Figure 8. Tiger salamander larvae collected in a seine haul ................................................... 14

\section{LIST OF TABLES}

Table 1. Impairment determinations from the MMI and O/E (RIVPACS) models................... 4

Table 2. Fish metrics and classification of fish species captured in the study area ...................5

Table 3. Species of vertebrates (fish and herpetofauna) collected during the survey

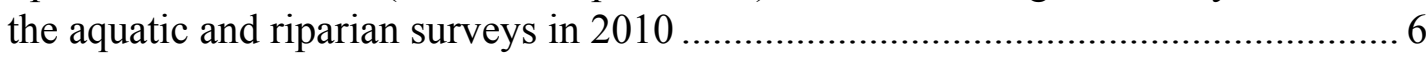

Table 4. Site habitat scores, functional assessment scores, fish IBI and

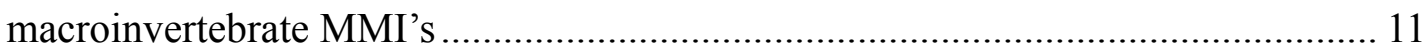

Table 5. Fish abundance, IBI"s and O/E results for the 12 sites reporting fish........................ 13 


\section{INTRODUCTION}

The Powder River basin in Wyoming and Montana is currently undergoing one of the world's largest coal bed natural gas (CBNG) developments. However, definitive information on the effects of CBNG product water on fish and aquatic invertebrates is lacking, making it difficult to predict the potential effects of this development on aquatic ecosystems (Davis et al. 2009). Therefore, pre-development baseline data and assessments of ecological condition are essential in assessing changes brought about by CBNG wells at the landscape or local reach scale. Despite numerous projects undertaken to document and monitor biological communities in the Powder and Tongue River watersheds (Confluence Consulting Inc. 2003, 2004; Stagliano 2006; Davis et al. 2009; Maxell 2009; Petersen et al. 2009; Senecal 2009), large gaps still exist in basic baseline surveys for riparian macroinvertebrate, fish, and herpetofauna. Many of the remaining gaps involve small prairie streams that constitute the Northwestern Great Plains Intermittent Prairie Stream ecological system (Stagliano 2005). This stream system may have downstream connectivity early in the season for potential fish spawning and nursery areas (Smith and Hubert 1989, Bramblett 2000, Trenka 2000) or no fish colonization at all, but by summer often becomes a string of isolated pools that are important breeding and rearing areas for amphibians. The largest unsurveyed landscapes containing this aquatic ecological system lie within the headwaters of Otter Creek (including Bear, Pasture and Bradshaw Creek) and upper Hanging Woman Creek (HWC) (including Trail and E.F. Trail Creek) (Figure 1). This area also contains proposed CBNG outfalls that could potentially be operational in the next year or two (MTDEQ, pers. comm.) (Figure 2). Identifying the presence of fish, macroinvertebrate, and herpetofauna that are Montana Species of Concern or BLM Sensitive Species prior to CBNG development is essential to understanding and potentially mitigating impacts to habitats and species. 


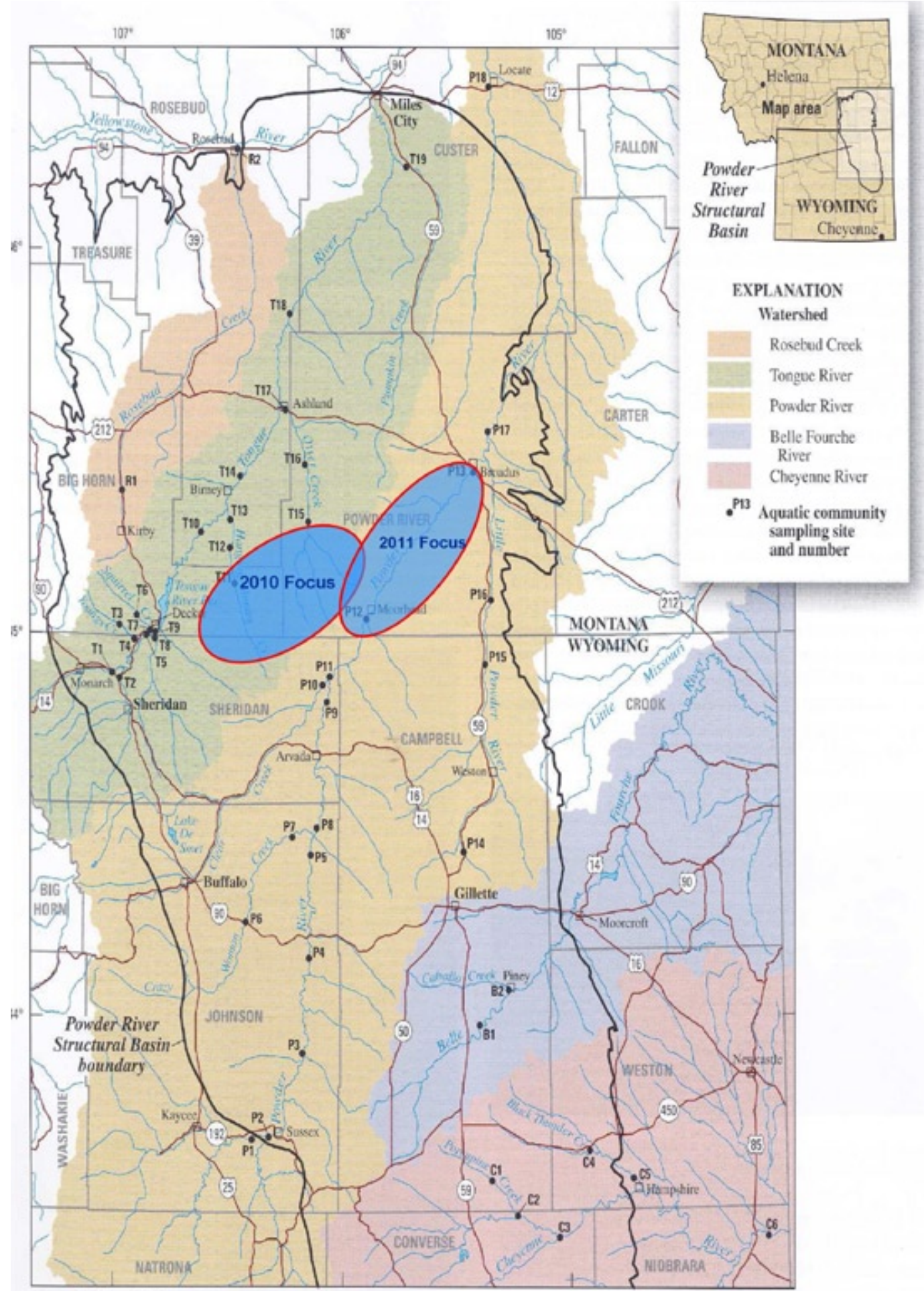

Figure 1. General overview of the project study area for the 2010 baseline assessments (USGS map). 


\section{Methods}

\section{Study Sites}

Prairie stream channels in the targeted watershed areas were accessed at legal right-of-ways, on BLM or state-owned parcels or with permission from landowners (specifically the Padlock, OW, and Forks Ranches). Sites were chosen strategically rather than randomly because we were targeting the presence of surface water, although larger stream-order dry channels were walked significant distances (up to $2 \mathrm{~km}$ ) to determine if isolated pools were present.

\section{Riparian Assessments}

Stream channels were walked in an upstream direction within the high water mark; GPS points were taken at any significant landform or channel change (e.g.,tributaries entering) until wetted pools were reached. The downstream pool was marked (GPS point) as the bottom of the reach and all ecological assessment protocols started from this point and continued upstream for $300 \mathrm{~m}$ (designated the assessment area or "AA"). Onsite habitat assessments were conducted using the rapid assessment protocol developed for the EPA by Barbour et al. (1999) with modifications for the BLM by the National Aquatic Assessment Team (scores 0-24) (http://www1.usu.edu/buglab/forms/ Bug\%20Protocol\%20form.pdf). The process for determining Proper Functioning Condition followed Pritchard et al. (1993). Using the BLM assessment protocols, the reach was divided into 10 equally spaced transects. Parameters recorded at each were: wetted width, bankfull width, 3 channel depth measurements, \% large woody debris and riparian shading. Basic water parameters (temperature, $\mathrm{pH}$, conductivity) were recorded prior to biological sampling using a Horiba H-10 water monitor, calibrated for the higher conductivity range. The Livestock Use Index ("Cowpie" CPI) was assessed at all streams by walking a randomly chosen $75 \mathrm{~m}$ reach on both sides of the stream channel in the riparian area within the assessment area and counting all the old and new cowpies (high CPI equals high cow usage). The goal of these evaluations is to characterize local reach geomorphology, riparian and in-stream habitat, and characteristics that influence aquatic community integrity. The sites ranking higher using these protocols are determined to have higher quality local-scale habitat. Habitat assessments were performed during the same visit as the biological sampling.

\section{Macroinvertebrate Communities}

Macroinvertebrates were collected from prairie stream pool sites with a modified EMAP ReachWide protocol (Lazorchak et al. 1998). Sampling involved multi-habitat, qualitative methods also outlined in the EPA protocols (Barbour et al. 1999). All habitats in the designated reach were apportioned along 10 transects and sampled qualitatively with a 500-micron D-frame net. A total of 10 habitat-weighted, randomized $1 \mathrm{~m}$ jabs or kicks were conducted within the reach, allowing 10 seconds per kick, and composited into one sample. All organisms in the net were washed on a 500-micron sieve, transferred to a one- liter Nalgene bottle, labeled and preserved in $95 \%$ ethanol and brought to the MTNHP lab in Helena for processing (sorting, identification and data analysis) by David Stagliano at the MNHP Helena lab following protocols used by the BLM Buglab: http:/www1.usu.edu/buglab/ process $/ 1$ ab\%20procedures.htm. These samples were collected within the MT DEQ recommend sampling time frame (June 1st-September 15th, (MTDEQ 2006). Macroinvertebrates were identified to the genus/species taxonomic level, counted and imported into EDAS (Jessup 2006), and biological metrics were calculated from the data using the Montana Department of Environmental Quality's newest multimetric macroinvertebrate (MMI) protocols (Jessup et al. 2005, Feldman 2006). Metric results were scored using the Montana DEQ bioassessment criteria and each sample categorized as nonimpaired or impaired according to threshold values (Table 1).

The macroinvertebrate MMI score is based upon a series of metrics that measure attributes of benthic macroinvertebrate communities that are sensitive to condition changes in a stream system (in the form of pollution or pollutants). The index score 
represents the condition of the macroinvertebrate community at the time the sample was collected within that past year. If the index score is below the impairment threshold, the individual metrics can be used to provide insight as to why the communities are different from the reference condition (Barbour et. al 1999, Jessup et. al. 2005). The impairment threshold set by MT DEQ is $\mathbf{3 7}$ for the Eastern Plains Stream Index, thus any scores above this threshold are considered unimpaired.

Table 1. Impairment determinations from the macroinvertebrate MMI and O/E (RIVPACS) models (taken from Jessup 2005, Feldman 2006).

\begin{tabular}{l|l|l|l} 
Ecoregion & RIVPACS & MMI & $\begin{array}{l}\text { Impairment } \\
\text { Determination }\end{array}$ \\
\hline Mountain & $\begin{array}{l}\geq 0.8 \text { or } \leq 1.2 \\
<0.8 \text { or }>1.2\end{array}$ & $\begin{array}{l}\geq 63 \\
<63\end{array}$ & $\begin{array}{l}\text { Not impaired } \\
\text { Impaired }\end{array}$ \\
Low Valley & $\geq 0.8$ or $\leq 1.2$ & $\geq 48$ & Not impaired \\
& $<0.8$ or $>1.2$ & $<48$ & Impaired \\
& & & \\
Eastern Plains & $\geq 0.8$ or $\leq 1.2$ & $\geq 37$ & Not impaired \\
& $<0.8$ or $>1.2$ & $<37$ & Impaired
\end{tabular}

\section{Fish and Amphibian Surveys}

Fish sampling in prairie stream pools was conducted with 20 foot straight seines in 25-30 meter increments within the $300 \mathrm{~m}$ stream channel assessment length following protocols outlined in Bramblett et al. (2005). Fish captured while seining were transferred to holding buckets until the reach was completed, unless the reach was broken up by dry or impassable sections; in this case, fish were worked up and released within the section of capture. Fish holding in the buckets were identified to species, enumerated, examined for external anomalies (e.g. deformities, eroded fins, lesions, and tumors), and then released. Young-of-the-year fish less than 20 millimeters (TL) were noted on the field sheet (not included in the totals) and released. Voucher specimens were only be taken in the case of uncertain field identifications. Adult amphibians or reptiles seen while seining or walking the designated stream reach were counted and recorded even if they were not captured in the seine.

Analysis of the sampled fish communities used Integrated Biotic Indices (IBI) (Bramblett et. al 2005) and derived Observed/Expected (O/E) Fish Models (Stagliano 2005) to detect impairment in the biological integrity of the sites. The IBI involved calculation of a series of metrics evaluating different attributes of the community (Table 2). The metrics allowed calculation of an overall score between 0 and 100. Bramblett et al. (2005) did not propose threshold criteria for good, fair, and poor biological integrity for these scores. Therefore, we applied commonly used criteria. Scores of 75 to 100 indicate good to excellent biological integrity, 50-74 fair to good biological integrity, 25 to 49 indicated poor to fair biological integrity and scores $<25 \%$ indicate poor biological integrity or severely impaired. 
Table 2. Fish metrics and classification of fish species captured in the 2010 ATG Study.

\begin{tabular}{|c|c|c|c|c|c|c|c|}
\hline Species & Scientific Name & Trophic* & $\begin{array}{l}\text { Feeding } \\
\text { Habitat } \uparrow\end{array}$ & $\begin{array}{l}\text { Repro } \\
\text { Guild } \$\end{array}$ & Tol** & Origin $\uparrow \dagger$ & $\begin{array}{l}\text { Total } \\
\text { Length } \\
3 \text { years }\end{array}$ \\
\hline \multicolumn{8}{|l|}{ Catostomidae } \\
\hline White sucker & Catostomus commersoni & $\mathrm{OM}$ & $\mathrm{BE}$ & LO & TOL & $\mathrm{N}$ & 229 \\
\hline \multicolumn{8}{|l|}{ Cyprinidae } \\
\hline Fathead Minnow & Pimephales promelas & $\mathrm{OM}$ & GE & TOL§ & TOL & $\mathrm{N}$ & 76 \\
\hline Brassy minnow & Hybognathus hankinsoni & HB & $\mathrm{BE}$ & & MOD & $\mathrm{N}$ & 94 \\
\hline Lake Chub & Couesius plumbeus & $\mathrm{OM}$ & GE & & MOD & $\mathrm{N}$ & 95 \\
\hline Common Carp & Cyprinus carpio & $\mathrm{OM}$ & $\mathrm{BE}$ & & TOL & I & 381 \\
\hline \multicolumn{8}{|l|}{ Centrarchidae } \\
\hline Green sunfish & Lepomis cyanellus & IC & GE & TOL $\S$ & TOL & I & 102 \\
\hline Pumpkinseed & Lepomis gibbosus & IC & GE & TOL $\S$ & TOL & I & 102 \\
\hline \multicolumn{8}{|c|}{ 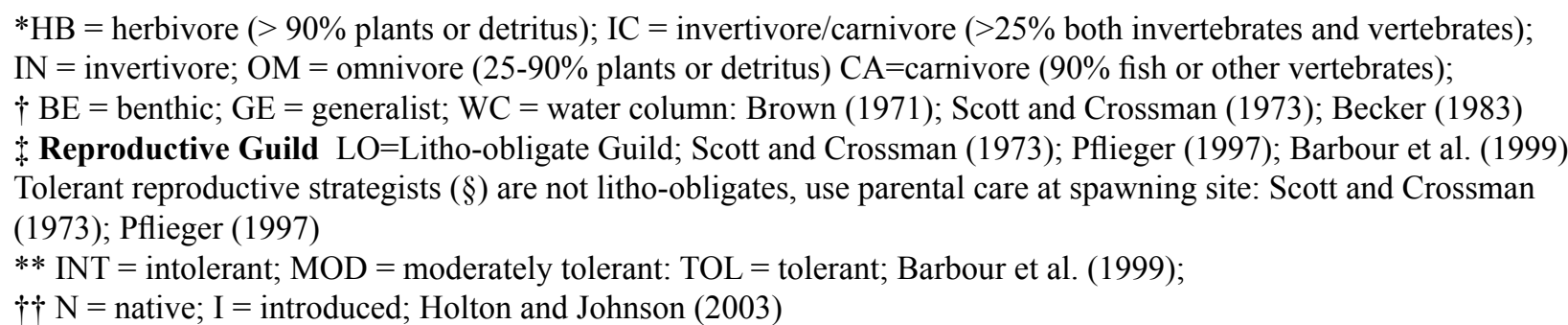 } \\
\hline
\end{tabular}




\section{Results}

We evaluated 87 stream reaches in the study area: 27 were dry reaches or ephemeral tributaries, 44 were interrupted pool reaches and 16 contained connected flowing water throughout the reach (Figure 2). Sixty stream reaches were seined or dip-netted for fish; only 12 sites contained fish, while 37 sites contained amphibians (Figure 4, Table 3). The majority of sites visited represented the Northwestern Great Plains Intermittent Prairie Stream ecological system (AES D005, E005), except the lowest sites on Otter and Hanging Woman Creek which could be described as Great Plains Perennial Prairie Stream (AES C005) (Stagliano 2005). The Intermittent Prairie Stream ecological system was fishless $80 \%$ of the time in this study; and therefore, represents an important breeding and rearing area for macroinvertebrates, amphibians and reptiles. Habitat assessments, water quality measurements, herpotofauna, macroinvertebrate and/or fish surveys were performed at 20 predetermined lotic (stream channel) reaches (per conversation with J. Chaffin). We have identified and characterized reference condition indicator assemblages for these ecosystems previously, and used these to compare our observed species sampled. These included sites on upper Otter and Hanging Woman Creeks, Trail and Bear Creeks and any tributaries containing suitable aquatic habitats.

\section{Habitat Evaluations}

Of 87 sampling reaches evaluated within the study area, we found 32 in Proper Functioning Condition (PFC) with a stable trend; 49 were Functional at Risk (FAR) with an undetermined trend, and 6 were ranked Nonfunctional (NF) (Figure $3 \&$ 4). In almost every case where a stream channel

Table 3. Vertebrates (species code) recorded during the ATG Riparian

Surveys. Frequency of Occurrence (FO) was calculated from the \# of wet sites $(n=60)$.

\begin{tabular}{|lcl|}
\hline Herpetofauna & $\begin{array}{c}\text { \# Sites } \\
\text { Present }\end{array}$ & FO \\
\hline Woodhouse's Toad (BUWO) & 37 & 0.62 \\
Tiger Salamander (AMTI) & 27 & 0.45 \\
Boreal Chorus Frog (PSMA) & 26 & 0.43 \\
Northern Leopard Frog (RAPI) & 12 & 0.20 \\
Painted Turtle (CHPI) & 4 & 0.07 \\
Terrestrial Gartersnake (THEL) & 2 & 0.03 \\
Western Rattlesnake (CRVI) & 2 & 0.03 \\
Eastern Racer (COCO) & 2 & 0.03 \\
Great Plains Toad (BUCO) & 1 & 0.02 \\
Plains Spadefoot Toad (SPBO) & 1 & 0.02 \\
Western Hognose Snake (HENA) & 1 & 0.02 \\
Plains Gartersnake (THRA) & 1 & 0.02 \\
Snapping Turtle (CHSE) & 1 & 0.02 \\
\hline Fish & & \\
\hline Fathead Minnow (FAMI) & 11 & 0.18 \\
Brassy Minnow (BRMI) & 7 & 0.12 \\
White Sucker (WHSU) & 4 & 0.07 \\
Lake Chub (LACH) & 3 & 0.05 \\
Common Carp* (CARP) & 1 & 0.02 \\
Green Sunfish* (GRSU) & 1 & 0.02 \\
Pumpkinseed* (PUMP) & 1 & 0.02 \\
\hline
\end{tabular}




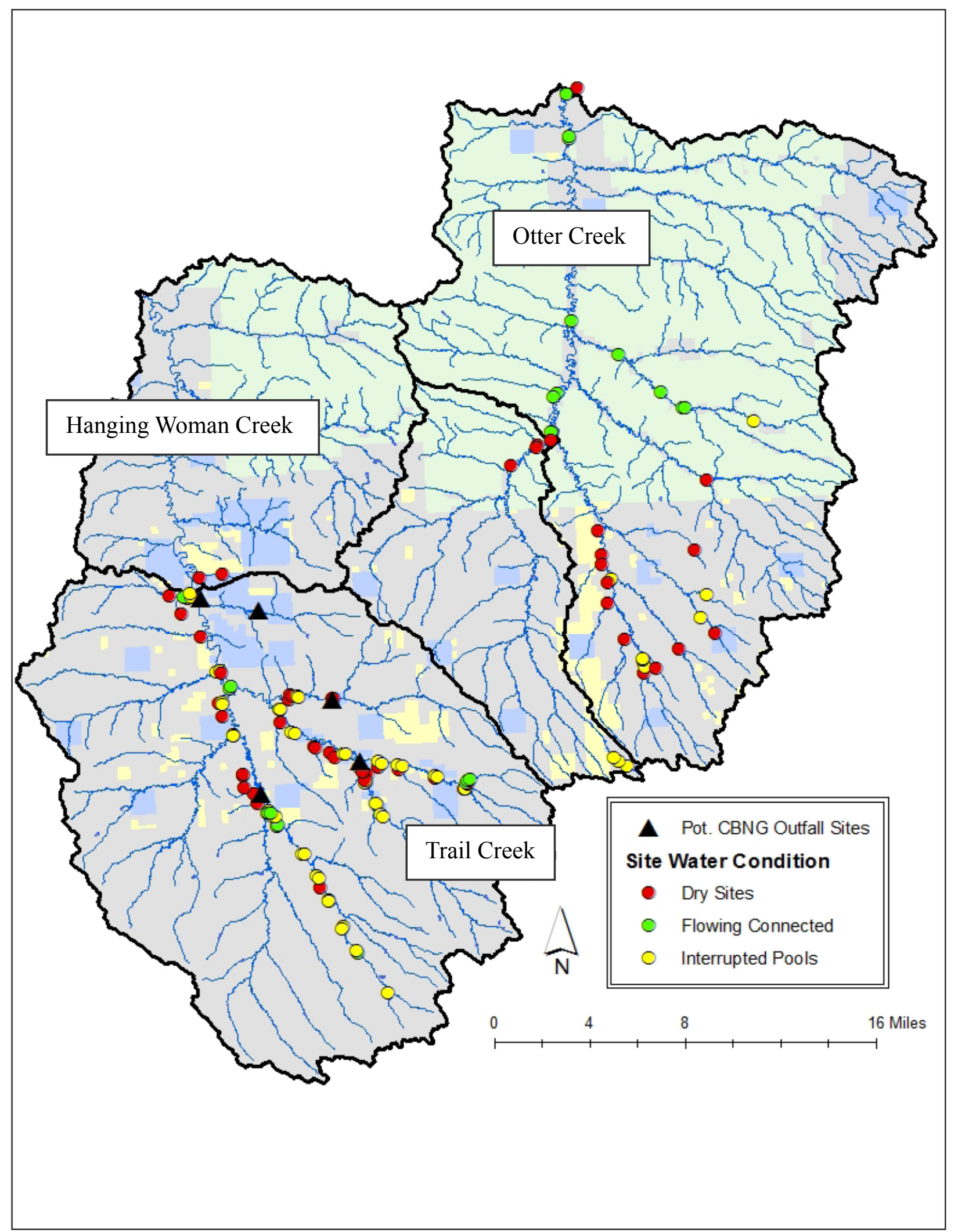

Figure 2. Stream Sites visited during the 2010 riparian assessments. (Red $=$ dry channels, Yellow $=$ Interrupted Pools, Green= Connected Flowing Water Reaches). 


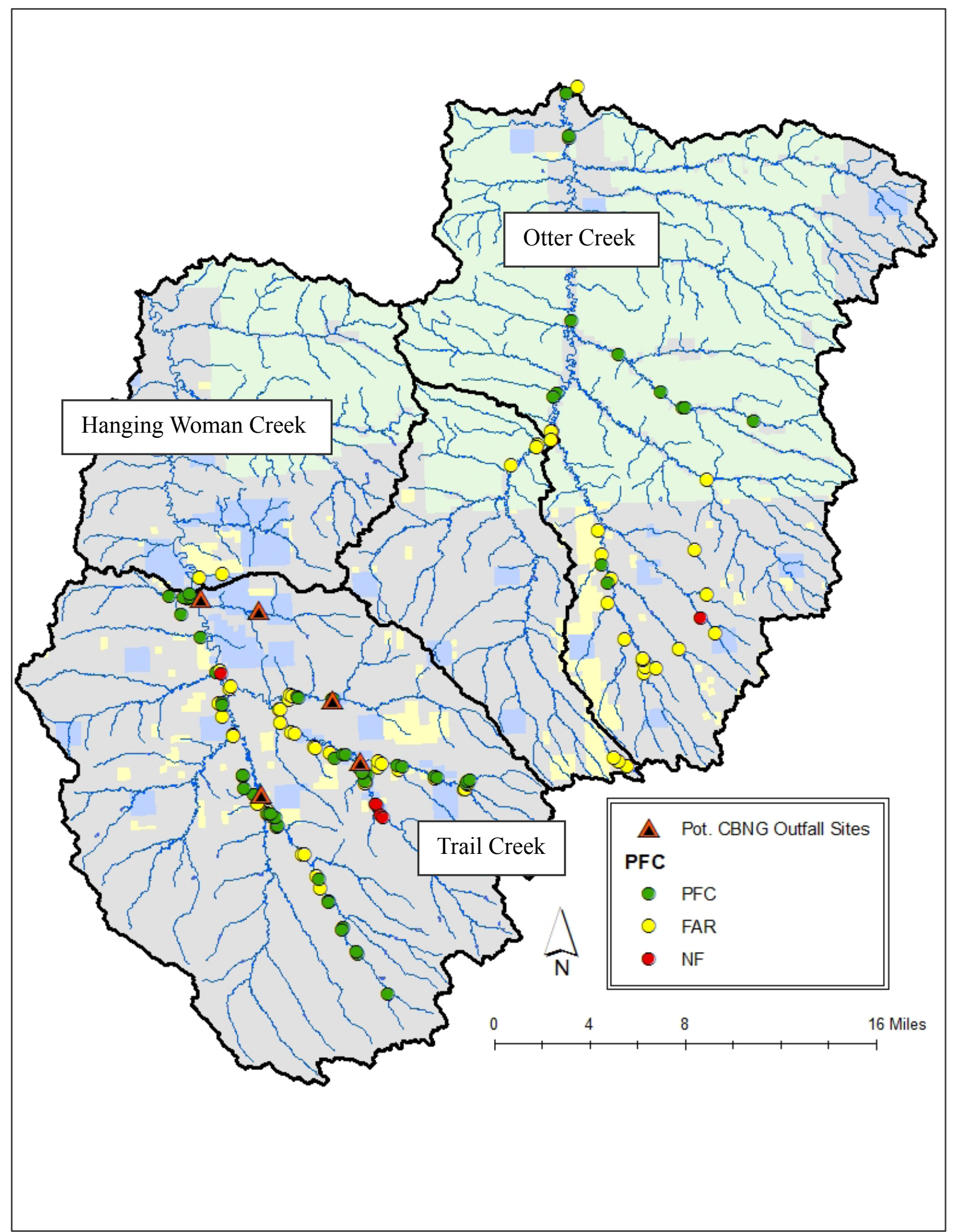

Figure 3. Riparian Functional Assessment Rankings for 2010 Reach Locations. PFC=Proper Functioning, FAR= Functional At Risk, NF=Non-functional. 
riparian area was assessed as FAR or NF, the reason was hydrologic modification, generally by livestock. In a few cases, inadequate or poorly functioning culverts or stream crossings were the cause of the hydrological impairment. Livestock (predominately cattle) grazing on open range is the dominant agricultural use in the assessment area. Grazing in riparian areas can cause stream and river bank destabilization, loss of riparian shade, and increased sediment and nutrient loads in the aquatic ecosystem (George et al. 2002). The livestock use index (CPI) largely correlated with the functional assessment rank with NF and some FAR reaches reporting high CPI (Appendix A). During our field surveys, we saw several instances where cattle had free access to riparian and wetlands areas, and some cases where pugging and hummocking had severely impacted both the soil and the vegetation (Figure 4). Highest site integrity scores using both BLM Buglab stream (24 max. score) and PFC habitat assessment methods were recorded at the Hanging Woman Creek sites ATG_143, a sedge meadow channel and ATG_141, a run-pool stream

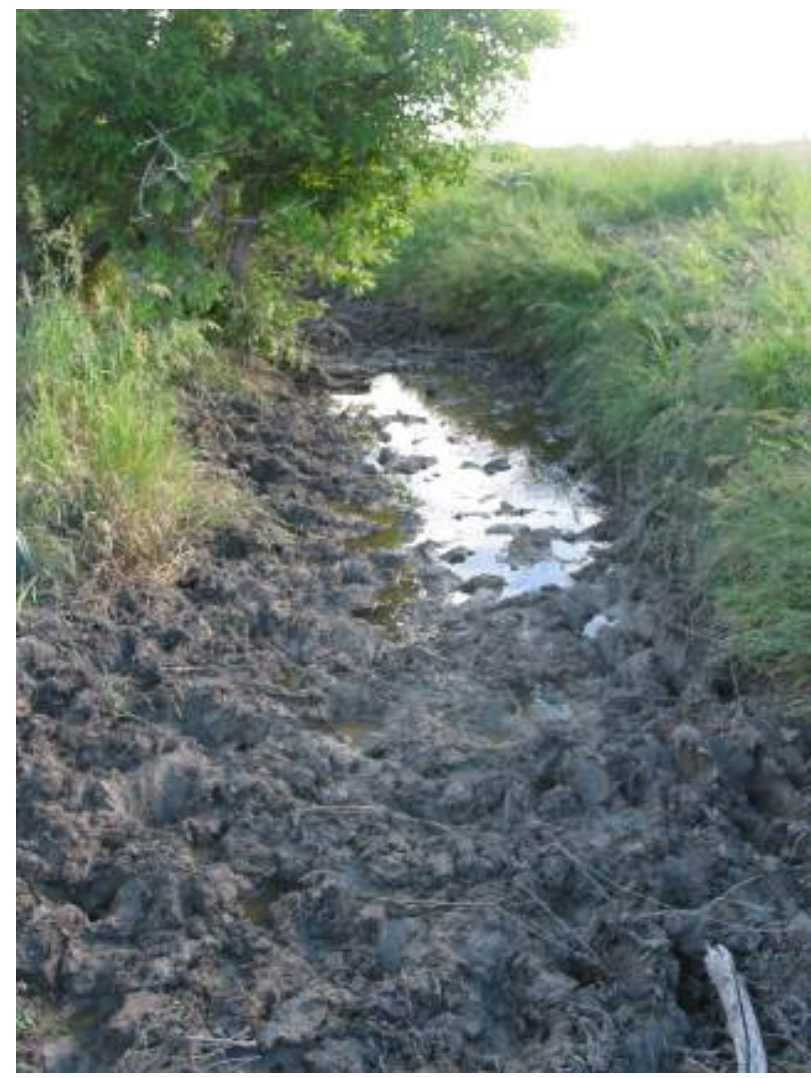

Figure 4. Photo of a structurally cattle-impacted, nonfunctional stream reach, site $A T G_{-} 158$. channel just upstream of the Montana border into Wyoming on the OW Forks Ranch. Other fairly intact sites were near the lower 76 Creek and Trail Creek confluence and upper East Fork Trail Creek. Sites with lower habitat scores were predominately degraded structurally by cattle usage and had associated high CPI values.

Other moderate integrity sites were near the 76 Creek and Trail Creek confluence, Otter Creek above Camp Creek and upper East Fork Trail Creek. The water quality parameters (conductivity $\&$ turbidity) taken at both Corral and upper Otter Creek sites were above the threshold for

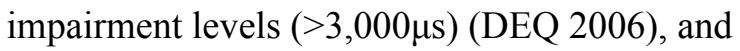
the Otter Creek site had visible signs of petroleum seepage from ground water.

\section{Macroinvertebrate Communities}

Overall, 110 unique macroinvertebrate taxa were reported from the 20 invertebrate collection sites (Appendix B). No macroinvertebrate species of concern (SOC) were collected. Average macroinvertebrate taxa richness per invertebrate site was 24.8 and the highest taxa richness reported at 2 Otter Creek sites was 36 taxa. Using the Montana DEQ macroinvertebrate multimetric index (MMI), 14 of the 20 sites were ranked nonimpaired (good to excellent biological integrity), 5 were marginally unimpaired and 1 slightly impaired (Table 4). Hanging Woman Creek sites \#143 \& \#196 had reference condition macroinvertebrate scores for a Great Plains Prairie Stream with DEQ MMI scores of 69.4 and 76.6, respectively. Most stream sites that contained flowing connected water scored higher with the MMI than sites with exclusively pool areas. Overall, sites evaluated in the Hanging Woman Creek basin received higher macroinvertebrate MMI scores than those in the Otter Creek basin, despite Otter Creek mainstem sites averaging higher taxa richness per site. The MT MMI was not significantly correlated with the riparian or in stream assessment scores (Figure 5). As mentioned in previous studies (Stagliano 2006), the MT MMI was not as effective at ranking expected macroinvertebrate communities from intact prairie intermittent sites that contained fairly intact communities because they were dominated by more tolerant species of that assemblage. 

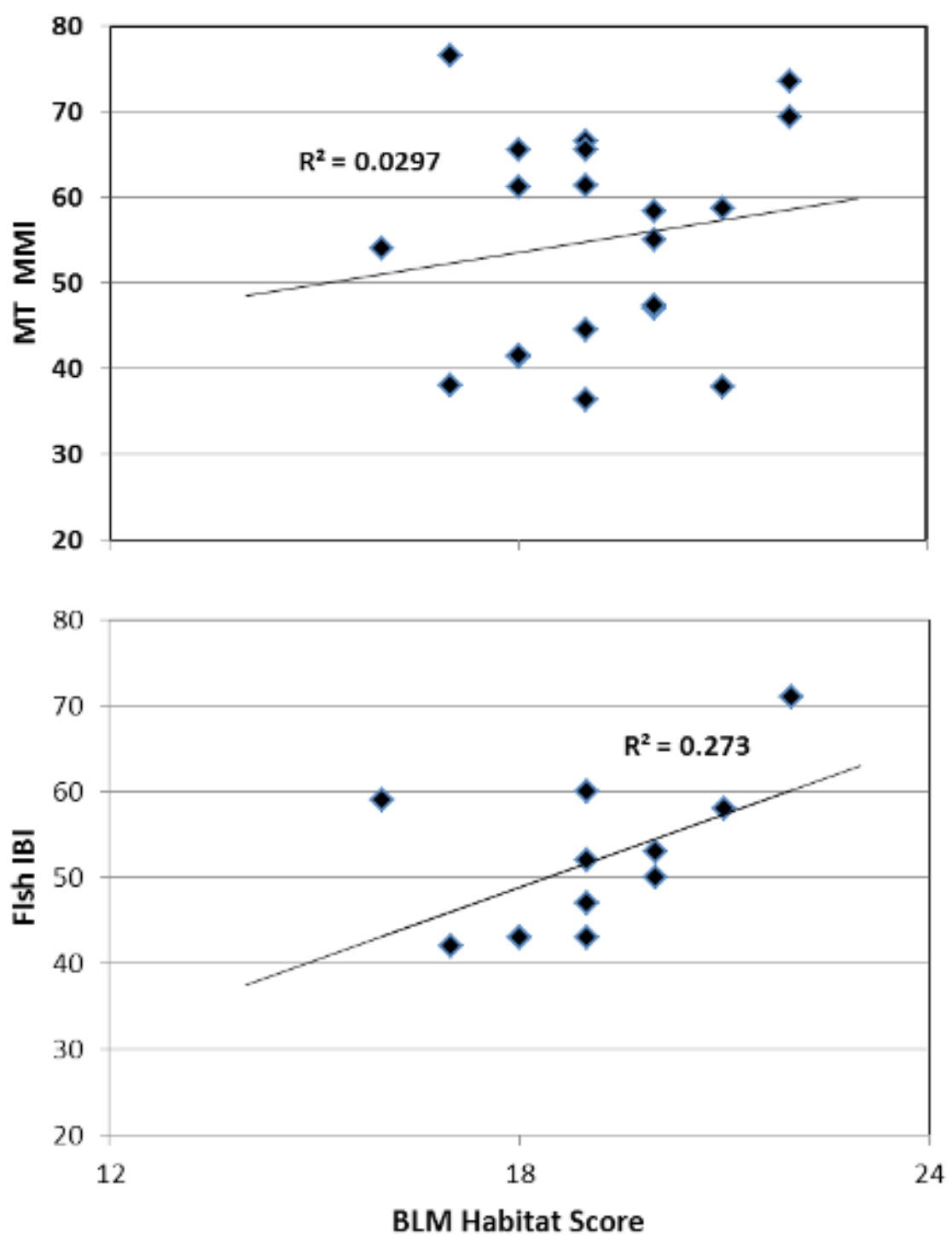

Figure 5. Macroinvertebrate MMI (top) and Fish IBI (bottom) Scores vs. BLM Stream Habitat Scores (max= 24) for 2010 ATG Sample Sites. 
Table 4. BLM Site Habitat Scores, Functional Assessment Scores, Fish IBI and Macroinvertebrate MMI's. Metric Score Ranks (+): unimpaired, (-): impaired, (=): marginal impairment, (na) = not applicable, taxa group not collected.

\begin{tabular}{|c|c|c|c|c|c|c|}
\hline Site Code & Site Name & $\begin{array}{c}\text { BLM Hab } \\
\text { Score } \\
\end{array}$ & \begin{tabular}{|c|} 
Riparian \\
PFC
\end{tabular} & Fish IBI & MT MMI & $\begin{array}{l}\text { Metric } \\
\text { Score } \\
\text { Ranks } \\
\end{array}$ \\
\hline ATG_124 & Waddle Creek OW Ranch \#1 & 16 & FAR & 64 & 54.0 & $=,+,+$ \\
\hline ATG_130 & Hanging Woman Creek uppermost site WY & 19 & PFC & no fish & 61.4 & $+, \mathrm{na},+$ \\
\hline ATG_132 & Hanging Wom an Creek OW Ranch county road & 20 & PFC & no fish & 47.1 & + ,na, + \\
\hline ATG_134 & Hanging Wom an Creek WP \#134 & 18 & PFC & no fish & 61.1 & $+, \mathrm{na},+$ \\
\hline ATG_137 & Hanging Wom an Creek WP \#137 & 21 & PFC & no fish & 58.7 &,+ na, + \\
\hline ATG_141 & Hanging Wom an Creek Forks Ranch WY border & 19 & PFC & 49 & 66.5 &,$+=,+$ \\
\hline ATG_143 & Hanging Wom an Creek Forks Ranch WY & 22 & PFC & no fish & 69.4 & + ,na, + \\
\hline ATG_145 & Hanging Wom an Creek Forks Ranch WY & 18 & PFC & 43 & 41.4 & $=,=,=$ \\
\hline ATG_150 & Hanging Wom an Creek Forks Ranch \#150 & 18 & PFC & 43 & 65.6 & $=,=,+$ \\
\hline ATG_151 & Hanging Wom an Creek Forks Ranch \#151 & 19 & PFC & 43 & 65.6 & $=,=,+$ \\
\hline ATG_160 & Seventysix Creek trib to 'Trail Creek & 20 & PFC & no fish & 47.4 & + ,na, + \\
\hline ATG_163 & Seventys ix Creek Lower trib to 'Trail Creek & 18 & PFC & no fish & 41.5 &,+ na, $=$ \\
\hline ATG_179 & Trail Creek \#179 & 17 & FAR & no fish & 38.0 & $=$,na, $=$ \\
\hline ATG_196 & Hanging Wom an Creek OW Ranch private bridg & 17 & FAR & 42 & 76.6 & $=,=,+$ \\
\hline ATG_200 & Hanging Woman Creek at Quietus Road Bridge & 20 & PFC & 53 & 55.0 &,,+++ \\
\hline ATG_201 & Otter Creek at Tenm ile Creek Road & 19 & PFC & 52 & 44.6 &,,$++=$ \\
\hline ATG_202 & Otter Creek at Taylor Creek Road & 19 & PFC & 60 & 36.3 &,,++- \\
\hline ATG_203 & Otter Creek at Cam p Creek Road & 20 & PFC & 50 & 58.4 &,,+++ \\
\hline ATG_235 & South Fork Taylor Creek & 22 & PFC & 71 & 73.5 &,,+++ \\
\hline ATG_CCC & Otter Creek at CCC Camp USFS & 19 & PFC & 58 & 37.9 &,,++- \\
\hline
\end{tabular}

\section{Fish Communities}

Overall, seven fish species (4 native/3 introduced, no SOCs) were identified from 1,219 individuals collected from 12 lotic ATG sites containing fish (Table 2, Table 3). All fish sites also reported amphibians present (Figure 6, Appendix A). Fathead minnows had the highest site occupancy rate of $92 \%$ (11 of 12 sites) and abundance, proportionally contributing $71 \%$ of all individuals collected (Table 3, Table 5). Brassy minnows were the next most collected species, occurring at 7 sites (Figure 7). The most diverse site was the Otter Creek site at the old CCC cabin with 5 species (4 native) and the most intact sites were Otter Creek at Taylor Creek and Fifteenmile Creek Roads each with 4 native species. Using Montana's Prairie Fish IBI, 4 of the 12 fish sites were ranked non-impaired (good biological integrity), 8 were slightly impaired (moderate integrity) and 2 were moderately impaired (poor-fair biotic integrity). No sites where fish were collected were ranked severely impaired by the Fish IBI, although some sites with no fish had habitat potential for fish to be present (permanent pools), but may have been limited by hydrologic connectivity to colonization sources either naturally (dry reaches) or man-induced (dams, stock ponds). The fish IBI community measure was more positively correlated to the BLM Habitat Score than the macroinvertebrate MMI (Figure 5).

Within the Great Plains Intermittent Prairie Streams, the species expected to occur most often at reference condition sites are the fathead minnow and brook stickleback (Table 5), but the upper Tongue and Powder River Basins are slightly out of the range of this species (brook stickleback); therefore, we did not collect them or expect them 


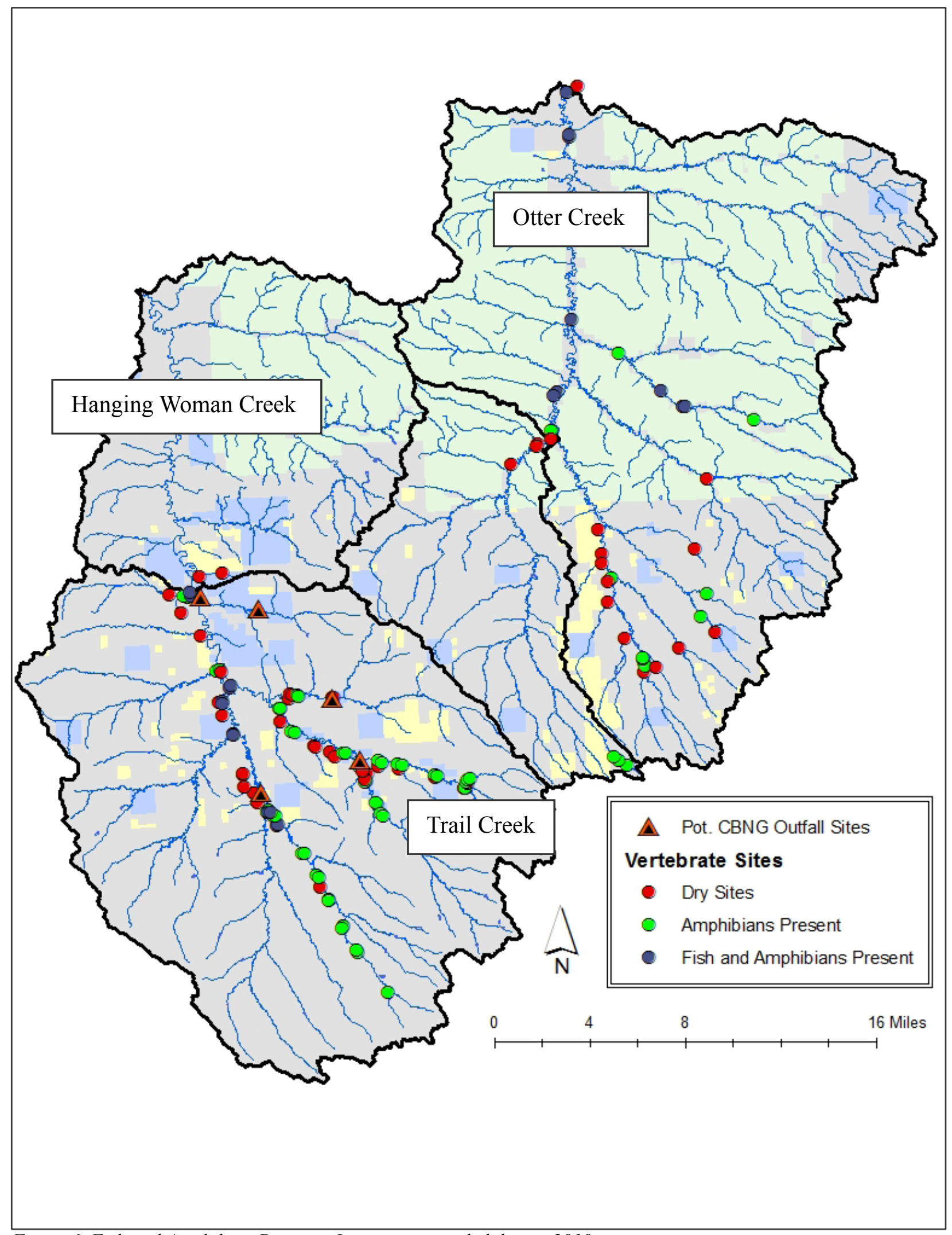

Figure 6. Fish and Amphibian Presence Locations recorded during 2010 surveys. 
Table 5. Fish abundance, IBI"s and O/E results for the 12 sites reporting fish.

\begin{tabular}{|c|c|c|c|c|c|c|c|c|c|c|c|c|}
\hline & 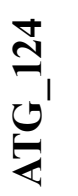 & 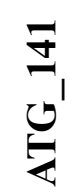 & 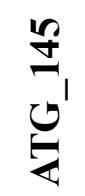 & $\begin{array}{l}\stackrel{8}{6} \\
\underset{6}{6}\end{array}$ & $\frac{\sqrt[n]{6}}{v^{\prime}}$ & 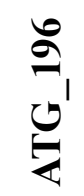 & 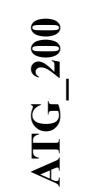 & 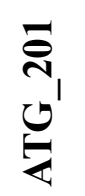 & 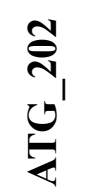 & 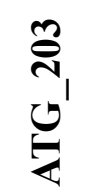 & 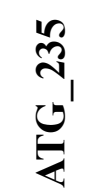 & 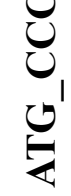 \\
\hline Brassy Minnow & 15 & 10 & 0 & 0 & 0 & 0 & 15 & 72 & 40 & 22 & 0 & 61 \\
\hline Common Carp* & 0 & 0 & 0 & 0 & 0 & 2 & 0 & 0 & 0 & 0 & 0 & 0 \\
\hline Lake Chub & 0 & 0 & 0 & 0 & 0 & 0 & 0 & 0 & 55 & 0 & 25 & 10 \\
\hline Fathead Minnow & 2 & 16 & 50 & 40 & 20 & 482 & 55 & 35 & 5 & 110 & 0 & 15 \\
\hline Green Sunfish* & 0 & 16 & 0 & 0 & 0 & 2 & 0 & 0 & 0 & 0 & 0 & 0 \\
\hline Pumpkinseed* & 0 & 0 & 0 & 0 & 0 & 0 & 0 & 0 & 0 & 0 & 0 & 4 \\
\hline White Sucker & 0 & 0 & 0 & 0 & 0 & 0 & 5 & 0 & 25 & 5 & 0 & 5 \\
\hline Total \# species & 2 & 3 & 1 & 1 & 1 & 3 & 3 & 2 & 4 & 3 & 1 & 5 \\
\hline Native Species & 2 & 2 & 1 & 1 & 1 & 1 & 3 & 2 & 4 & 3 & 1 & 4 \\
\hline Total Individuals & 17 & 42 & 50 & 40 & 20 & 486 & 75 & 107 & 125 & 137 & 25 & 95 \\
\hline IBI & $\underline{64}$ & 49 & 43 & 43 & 43 & 42 & 53 & 52 & $\underline{60}$ & 50 & 71 & $\underline{58}$ \\
\hline $\mathbf{O} / \mathbf{E}$ & 0.82 & 0.82 & 0.41 & 0.41 & 0.41 & 0.41 & 1.22 & 0.82 & $\overline{1.63}$ & 1.22 & 1.25 & 0.57 \\
\hline & 81.6 & 81.6 & 40.8 & 40.8 & 40.8 & 40.8 & 122.4 & 81.6 & 163.3 & 122.4 & 125.0 & 57.1 \\
\hline
\end{tabular}

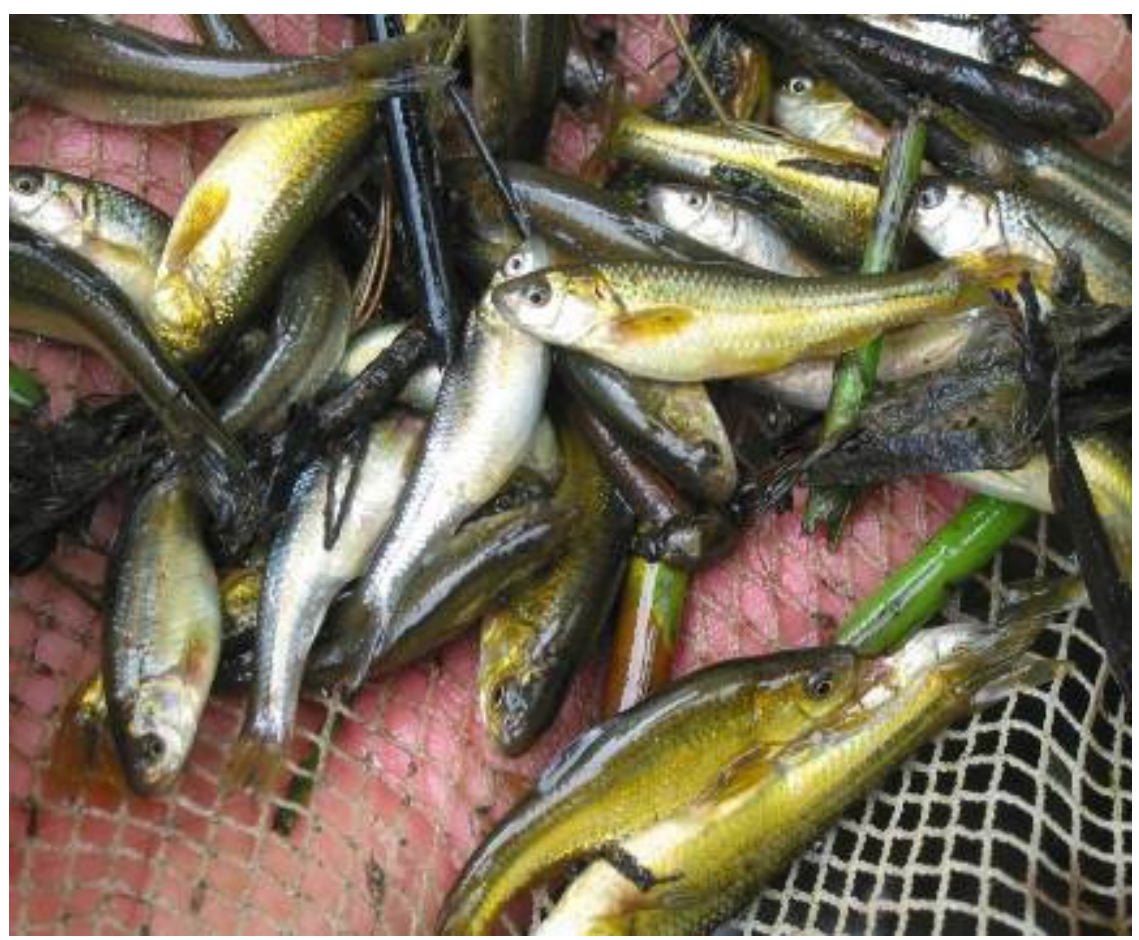

Figure 7. Photo of brassy minnows in spawning colors and white sucker (ATG CCC) 
to occur in these sites and revised the Observed / Expected (O/E) Fish Model predictors accordingly. Using Montana's Prairie Fish IBI of the 12 fish sites were ranked non-impaired (good to excellent biological integrity), 6 were slightly impaired (moderate integrity) and 5 moderately impaired (poor biotic integrity) (Table 4). No sites were ranked severely impaired by the Fish IBI. The O/E scores agreed with the IBI scores in most cases, except for the larger stream reaches that could be almost classified as Perennial Prairie Streams (ATG_CCC) (Table 4); thus, the O/E would expect more fish species to be present than are actually observed causing an artificially low score. Otter Creek CCC site was ranked unimpaired/slightlyimpaired by the IBI, but the $\mathrm{O} / \mathrm{E}$ ranked this site as moderately impaired $(\mathrm{O} / \mathrm{E}=57.6)$, because it would be missing almost $43 \%$ of the expected taxa.

\section{Amphibian and Reptile Communities}

A total of 13 herpetofauna species were recorded across the study region and at least one amphibian species was present at $62 \%$ of wetted sites, while no occurrences were recorded surveying dry reaches (Table 3). Upper Trail Creek near Spring Creek (ATG_176) had the highest site herpetofauna species richness with five species, while 25 sites reported three commonly associated species (Woodhouse's Toad, Bufo woodhousii, Tiger Salamander, Ambystoma tigrinum (Figure 8) and Boreal Chorus Frog, Psuedacris maculata). Woodhouse's Toad had the highest site occupancy, occurring at 37 of 60 sites, followed by the Tiger Salamander and Boreal Chorus Frog recorded at 27 and 26 sites, respectively (Table 3). The Northern Leopard Frog, Rana pipiens occurred at 12 sites and was more commonly associated with sites that also contained fish (8 co-occurring sites). Plains Spadefoot Toad, Spea bombifrons (SOC), and the Great Plains Toad, Bufo cognatus) were recorded at one site each in the Trail Creek and Upper Hanging Woman Creek drainages. Seven reptile specieswere recorded during the surveys (in order of site occurrence: Painted Turtle, Chrysemys picta, Terrestrial Garter Snake, Thamnophis elegans Western Rattlesnake, Crotalus viridis; Plains Garter Snake, Thamnophis radix; Western Hognose Snake, Heterodon nasicus (SOC) and Snapping Turtle, Chelydra serpentina (SOC (Table 3).

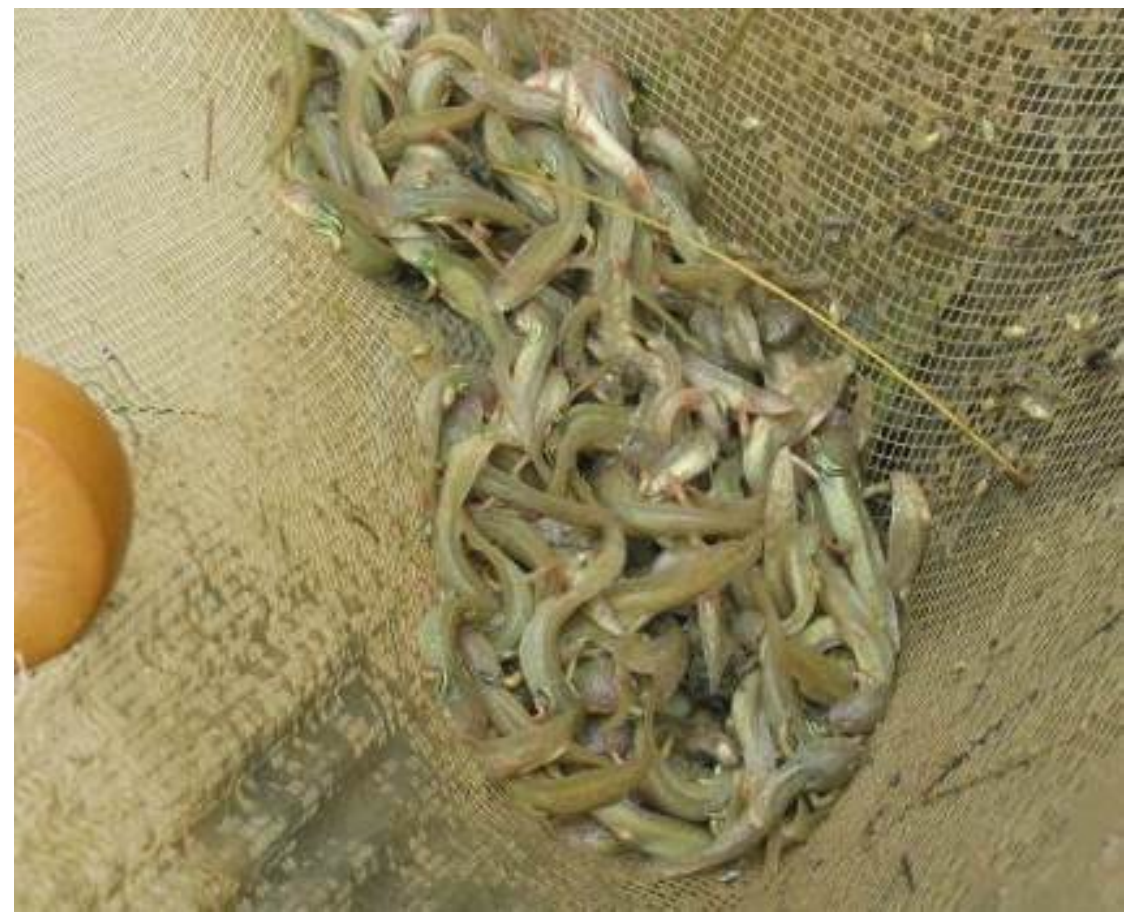

Figure 8. Seine haul of Tiger Salamander Larvae (60-70 $\mathrm{mm}$ SLV) at site $A T G_{-} 198$. 


\section{Conclusions and Recommendations}

Diverse aquatic communities were identified at the majority of intermittent aquatic sites in the upper HWC and Otter Creek region, despite most sites lacking fish communities. Stream sites visited in the HWC basin had higher than average landscape integrity reflected at the local reach-scale with high biological and riparian integrity. HWC consistently scored higher with the macroinvertebrate community metrics, while Otter Creek scored better with the fish community metrics. Threats to this ecological system that can be managed include grazing and livestock use around the riparian areas. This occurs moderately in these basins and can have strong local effects resulting in sedimentation, stream widening at cattle crossings and loss of functional channel hydrology. Introductions of game (green sunfish, bullheads or pumpkinseeds) or forage fish in stock ponds anywhere in the watershed can pose potential problems for native fish as these introduced fish become permanent residents and either outcompete or prey upon the native fish communities. These prairie river ecological types are abundant in terms of river miles across the Northern Great Plains of western North America, but the hydrology (i.e. water permanence) can be easily affected by upstream dams or diversions in the watershed. Stock ponds that are stocked with game fish, and bait bucket introductions can also contribute to the community degradation brought about by the introduced fish species. Diverse communities with high biological integrity are highly correlated with good riparian condition and high habitat quality. Thus, effective riparian zone management in the grazing of cattle will contribute to intact vegetation buffers and less sediment in the aquatic environment. Macroinvertebrate communities ranked few sites as impaired, even those with an impaired riparian condition, but the effectiveness of macroinvertebrates in assessing prairie streams is still under debate in Montana.
Community results from the habitat, fish and macroinvertebrate surveys combined to rank the following sites from highest biological integrity to lowest within their aquatic ecological classification codes:

Northwestern Great Plains Perennial Prairie

Stream (AES code C005)

1) Hanging Woman Creek (Quietus Road, OW

Ranch)

2) Otter Creek CCC camp site

Northwestern Great Plains Intermittent Prairie Stream (AES code D005)

1) Otter Creek at Camp Creek Road

2) South Fork Taylor Creek

3) Hanging Woman Creek \#141

4) Waddle Creek OW Ranch \#1

5) HWC \#150, HWC \#151

6) $\mathrm{HWC} \# 202$

7) HWC \#201

\section{Great Plains Intermittent Fishless Prairie} Stream (AES code E005)

1) Hanging Woman Creek \#143

2) Hanging Woman Creek \#130

3) HWC \#137

4) HWC \#134

5) Seventysix Creek \#160

6) HWC \#132

7) Seventysix Creek \#163

8) Trail Creek \#179

Northwestern Great Plains Perennial Spring (AES code S005)

1) Upper Trail Creek Spring 


\section{Literature Cited}

Allan, J. D., D. L. Erickson and J. Fay. 1997. The influence of catchment land use on stream Integrity across multiple spatial scales. Freshwater Biology 37:149-162.

Barbour, M., J. Gerritsen, B.D. Snyder, and J.B. Stribling. 1999. Rapid Bioassessment Protocols for Use in Streams and Wadable Rivers: Periphyton, Benthic Macroinvertebrates and Fish, Second Edition. EPA 841-B-99-002. United States Environmental Protection Agency; Office of Water: Washington, D.C.

Bramblett, R. G., T. R. Johnson, A. V. Zale, A. V., and D. Heggem. 2005. Development and Evaluation of a Fish Assemblage Index of Biotic Integrity for Northwestern Great Plains. Transactions of the American Fisheries Society 134:624-640, 2005.

Bramblett, R. G., and K. D. Fausch. 1991. Variable fish communities and the index of biotic integrity in a western Great Plains river. Transactions of the American Fisheries Society 120:752-769.

Dodds, W. K., K. Gido, M. R. Whiles, K. M. Fritz, and W. J. Matthews. 2004. Life on the edge: The ecology of Great Plains prairie streams. BioScience 54: 205-216.

Feldman, D. 2006. Interpretation of New Macroinvertebrate Models by WQPB. Draft Report. Montana Department of Environmental Quality, Planning Prevention and Assistance Division, Water Quality Planning Bureau, Water Quality Standards Section. 1520 E. 6th Avenue, Helena, MT $59620.14 \mathrm{pp}$.
George, M.R., R.E. Larsen, N.K. McDougald, K.W. Tate, J.D. Gerlach, Jr., and K.O. Fulgham. 2002. Influence of grazing on channel morphology of intermittent streams. J. Range Management. 55:551-557.

Hawkins, C. P. and R. H. Norris. 2000. Performance of different landscape classifications for aquatic bioassessments: introduction to the series. Journal of the North American Benthological Society.19:3 (367-369).

Holton, G. D., and H. E. Johnson. 2003. A field guide to Montana fishes, 3rd edition. Montana Fish, Wildlife, and Parks, Helena.

Jessup, B., J. Stribling; and C. Hawkins. 2005. Biological Indicators of Stream Condition in Montana Using Macroinvertebrates. Tetra Tech, Inc. November 2005 (draft).

Jessup, B. 2006. Ecological Data Application System (EDAS) Version MT 3.3.2k A User's Guide. Tetra Tech, Inc.

Lazorchak, J.M., Klemm, D.J., and D.V. Peck (editors). 1998. Environmental Monitoring and Assessment Program - Surface Waters: Field Operations and Methods for Measuring the Ecological Condition of Wadeable Streams. EPA/620/R-94/004F. U.S. Environmental Protection Agency, Washington, D.C.

Montana Department of Environmental Quality (DEQ). 2006. Sample Collection, Sorting, and Taxonomic Identification of Benthic Macroinvertebrates. Water Quality Planning Bureau. Standard Operation Procedure (WQPBWQM-009). 
Peterson, D.A., Clark, M.L., Foster, Katharine, Wright, P.R., and Boughton, G.K., 2010, Assessment of ecological conditions and potential effects of water produced from coalbed natural gas development on biological communities in streams of the Powder River structural basin, Wyoming and Montana, 2005-08: USGS Scientific Investigations Report 2010-5124, 84 p. http://pubs.usgs.gov/sir/2010/5124/pdf/ SIR10-5124.pdf

Pritchard, D., F. Barret, H. Berg, W. Hagenbuck, R. Krapf, R. Leinard, S. Leonard, M. Manning, C. Noble, Tippy, D. 1993. Riparian Area Management: A User Guide To Assessing Proper Functioning Condition. Technical Reference 1737-9. USDI Bureau of Land Management Service Center. Denver, Colorado. USA. 109 pp.

Relyea, C., G. Minshall, Robert Danehy. 2000. Stream Insects as Bioindicators of Fine Sediment, Stream Ecology Center, Department of Biological Sciences, Idaho State University. Watershed Management 2000 Conference.

Stagliano, David, M. 2005. Aquatic Community Classification and Ecosystem Diversity in Montana's Missouri River Watershed. Report to the Bureau of Land Management. Montana Natural Heritage Program, Helena, Montana. 65 pp. plus appendices. http://www.mtnhp.org/reports. asp\#Ecology 

Appendix A. All Sites Visited and Site Information Collected During the 2010 ATG Riparian Assessments 



\begin{tabular}{|c|c|c|c|c|c|c|c|c|c|c|c|c|c|c|c|c|c|c|c|c|c|c|c|c|c|c|c|}
\hline$\overline{\mathrm{u}}$ & 0 & 6 & $\sim \mid$ & 0 & 0 & 0 & - & $\nabla$ & $\nabla$ & 0 & $\nabla$ & $\nabla$ & $\nabla$ & $\nabla$ & $\sim$ & $\sim \mid$ & $\sim$ & $m$ & $m$ & 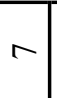 & $r$ & $=$ & $=$ & $\infty$ & $\infty$ & $\sim$ & $\sim$ \\
\hline$\frac{U}{G}$ & 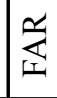 & $\underset{\text { 寽 }}{2}$ & 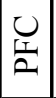 & 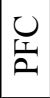 & \begin{tabular}{|l}
0 \\
II \\
L
\end{tabular} & 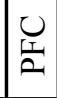 & $\begin{array}{l}0 \\
\text { II } \\
2\end{array}$ & $\underset{\text { 茎 }}{2}$ & 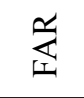 & $\begin{array}{l}0 \\
\text { II } \\
2\end{array}$ & U & 足 & 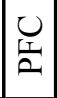 & 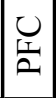 & 足 & 足 & 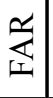 & 䍃 & 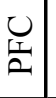 & 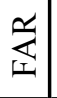 & 龸 & 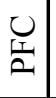 & 吉 & 足 & 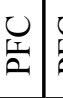 & $\begin{array}{l}U \\
\mathrm{~L} \\
\mathrm{~L}\end{array}$ & 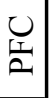 \\
\hline$\sum_{\Delta} \frac{0}{\underline{I}}$ & 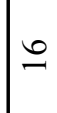 & $\because$ & $\infty$ & $\stackrel{\infty}{-}$ & 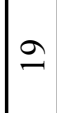 & $\cong$ & $\stackrel{2}{=}$ & $\stackrel{\bullet}{0}$ & ○ & $\stackrel{\infty}{\sim}$ & 2 & $\curvearrowright$ & ㄱ. & 이 & $\infty$ & $\infty$ & 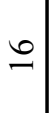 & $\vec{\sim}$ & $\vec{\sim}$ & ㄱ. & શે. & 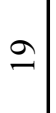 & 9 & $\approx$ & $\approx$ & $\infty$ & 9 \\
\hline 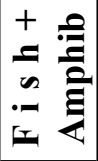 & - & - & 0 & 0 & 0 & 0 & 10 & 0 & 0 & 0 & 10 & 0 & 0 & 0 & 0 & 0 & 0 & 0 & 0 & 0 & 0 & -1 & - & 0 & 0 & - & - \\
\hline 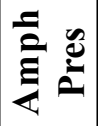 & - & - & 0 & 0 & 10 & 0 & 0 & - & - & - & - & - & -1 & -1 & - & - & 0 & -1 & - & - & - & -1 & - & - & - & - & - \\
\hline$\stackrel{\Xi}{\infty}$ & - & - & 0 & 0 & 0 & 0 & 10 & 0 & 0 & 0 & 10 & 0 & 0 & 0 & 0 & 0 & 0 & 0 & 0 & 0 & 0. & -1 & - & 0 & 0 & - & - \\
\hline 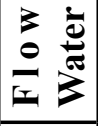 & 0 & 0 & 0 & 0 & 10 & 0 & 10 & 0 & 0 & 0 & - & 0 & 0 & 10 & 0 & 0 & 0 & 0 & 0 & 0 & 0. & -1 & - & 0 & 0 & - & - \\
\hline $\begin{array}{l}\grave{D} \\
\stackrel{口}{\Xi} \\
\Xi\end{array}$ & - & - & 0 & 0 & 0 & 0 & 10 & - & - & - & 10 & - & -7 & - & - & - & 0 & -1 & - & -1 & -1 & 0 & 0 & - & - & 0 & 0 \\
\hline$\vec{\theta}$ & 0 & 0 & -1 & - & - & - & - & 0 & 0 & 0 & 0 & 0 & 0 & 0 & 0 & 0 & -1 & 0 & 0 & 0 & 0 & 0 & 0 & 0 & 0 & 0 & $o$ \\
\hline 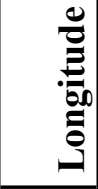 & 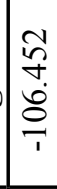 & 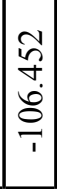 & 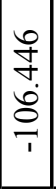 & $\begin{array}{l}\hat{m} \\
\stackrel{\sigma}{\circ} \\
\stackrel{0}{0} \\
\stackrel{1}{1}\end{array}$ & 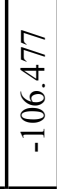 & \begin{tabular}{l}
$n$ \\
\multirow{f}{*}{} \\
$\stackrel{0}{0}$ \\
\hdashline \\
1
\end{tabular} & $\mid$\begin{tabular}{l}
0 \\
\multirow{+}{+}{} \\
$\dot{0}$ \\
0 \\
1
\end{tabular} & $\begin{array}{l}\stackrel{2}{7} \\
\stackrel{+}{\circ} \\
\frac{1}{1}\end{array}$ & $\begin{array}{l}\stackrel{7}{+} \\
\stackrel{+}{\circ} \\
\stackrel{8}{1}\end{array}$ & 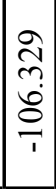 & 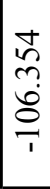 & $\begin{array}{l}n \\
n \\
n \\
0 \\
0 \\
1\end{array}$ & $\left|\begin{array}{l}0 \\
0 \\
n \\
0 \\
0 \\
1 \\
1\end{array}\right|$ & $\mid \begin{array}{l}0 \\
0 \\
n \\
0 \\
0 \\
1 \\
1\end{array}$ & \begin{tabular}{c}
$\hat{0}$ \\
$\hat{n}$ \\
$\stackrel{0}{0}$ \\
\hdashline \\
1
\end{tabular} & $\begin{array}{c}0 \\
2 \\
n \\
0 \\
0 \\
1 \\
1\end{array}$ & 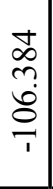 & $\left|\begin{array}{c}0 \\
\infty \\
m \\
ٌ \\
0 \\
1 \\
1\end{array}\right|$ & 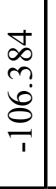 & 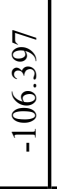 & $\mid \begin{array}{c}n \\
\hat{n} \\
\stackrel{0}{0} \\
0 \\
1\end{array}$ & $\begin{array}{l}\stackrel{\vartheta}{\vec{\nabla}} \\
\stackrel{0}{0} \\
\stackrel{0}{1}\end{array}$ & 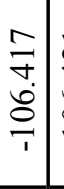 & $\begin{array}{l}\vec{~} \\
\stackrel{+}{\circ} \\
\stackrel{\circ}{0} \\
\stackrel{1}{1}\end{array}$ & 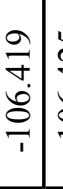 & 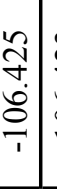 & $\begin{array}{l}\stackrel{\text { }}{f} \\
\stackrel{0}{\circ}\end{array}$ \\
\hline & $\begin{array}{l}\stackrel{g}{0} \\
\dot{f} \\
\dot{f}\end{array}$ & $\begin{array}{l}q \\
0 \\
\dot{g} \\
\dot{\sigma}\end{array}$ & \begin{tabular}{l}
\multirow{J}{*}{} \\
$\vdots$ \\
$\dot{v}$ \\
$\forall$
\end{tabular} & $\begin{array}{l}m \\
0 \\
\dot{\sigma} \\
\dot{\gamma}\end{array}$ & 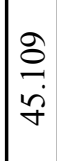 & $\begin{array}{l}- \\
0 \\
\dot{y} \\
\gamma\end{array}$ & 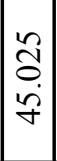 & $\begin{array}{l}\widetilde{8} \\
8 \\
\dot{\gamma}\end{array}$ & $\begin{array}{l}\overline{8} \\
\dot{8} \\
\dot{\gamma}\end{array}$ & $\begin{array}{l}2 \\
\infty \\
\dot{f} \\
\dot{J}\end{array}$ & $\begin{array}{l}\vec{\sigma} \\
\dot{\sigma} \\
\dot{f}\end{array}$ & $\begin{array}{l}\frac{n}{\sigma} \\
\dot{f}\end{array}$ & 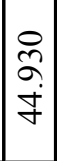 & 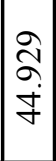 & $\begin{array}{l}\hat{J} \\
\stackrel{f}{f} \\
\dot{f}\end{array}$ & $\begin{array}{l}\dot{0} \\
\dot{+} \\
\dot{J} \\
\dot{f}\end{array}$ & $\begin{array}{l}\dot{+} \\
2 \\
\dot{z}\end{array}$ & 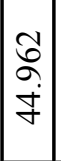 & $\begin{array}{l}8 \\
8 \\
ٌ \\
\dot{\nabla}\end{array}$ & $\begin{array}{l}n \\
\hat{a} \\
\dot{f}\end{array}$ & $\begin{array}{l}n \\
\hat{a} \\
\dot{y} \\
\dot{f}\end{array}$ & $\begin{array}{l}\hat{\sigma} \\
\dot{\nabla}\end{array}$ & $\begin{array}{l}\text { } \\
\dot{f}\end{array}$ & $\begin{array}{l}\text { बे } \\
\text { ஓं }\end{array}$ & $\begin{array}{l}\text { } \\
\text { jे }\end{array}$ & $\begin{array}{l}\overrightarrow{8} \\
8 \\
\dot{\gamma} \\
\forall\end{array}$ & $\begin{array}{l}\overline{8} \\
\text { ij }\end{array}$ \\
\hline 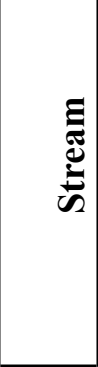 & 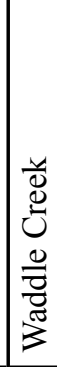 & 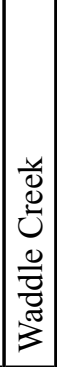 & 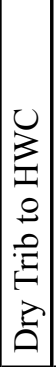 & $\begin{array}{l}0 \\
3 \\
1 \\
0 \\
0 \\
0 \\
0 \\
1 \\
0 \\
0 \\
0\end{array}$ & 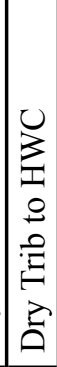 & \begin{tabular}{|l}
0 \\
3 \\
3 \\
0 \\
0 \\
0 \\
0 \\
0 \\
0 \\
0 \\
0
\end{tabular} & $\mid \begin{array}{l}0 \\
3 \\
1 \\
0 \\
0 \\
0 \\
0 \\
1 \\
0 \\
0 \\
0\end{array}$ & 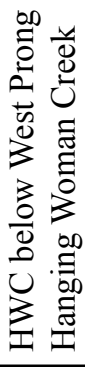 & 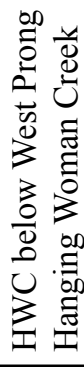 & 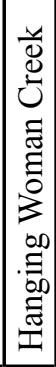 & 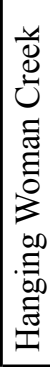 & 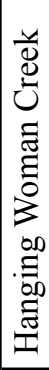 & 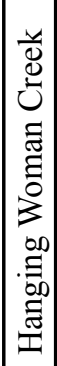 & 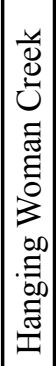 & 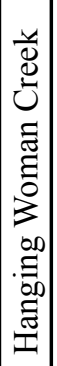 & 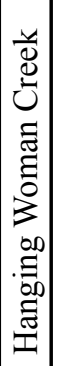 & 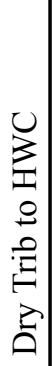 & 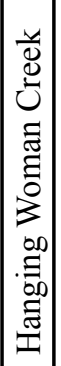 & 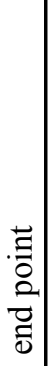 & 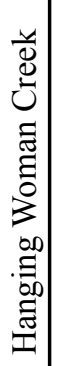 & 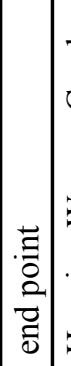 & 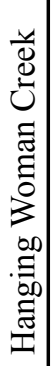 & 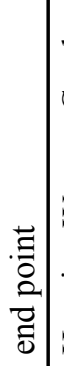 & 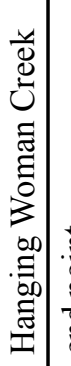 & 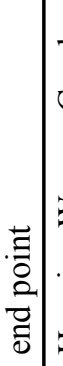 & 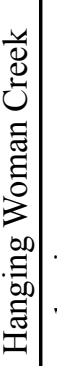 & 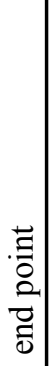 \\
\hline 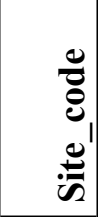 & 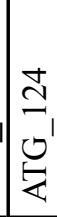 & 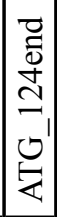 & 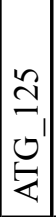 & 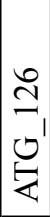 & 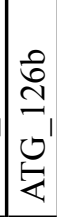 & 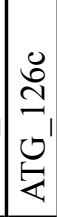 & 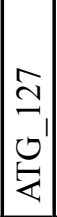 & 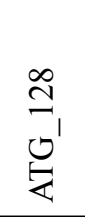 & 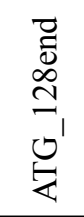 & 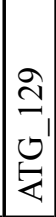 & 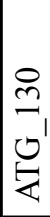 & 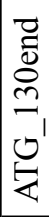 & 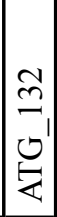 & 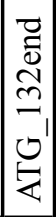 & 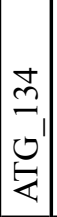 & 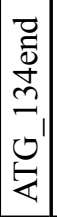 & $\begin{array}{l}0 \\
2 \\
0 \\
0 \\
\\
\end{array}$ & 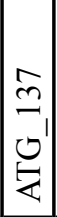 & 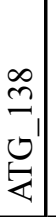 & 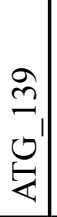 & 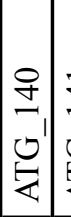 & 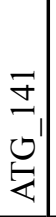 & \begin{tabular}{l}
$\mathcal{Y}$ \\
\multirow{J}{*}{} \\
安
\end{tabular} & \begin{tabular}{l}
$\stackrel{3}{ \pm}$ \\
\multirow{U}{}{} \\
安
\end{tabular} & $\begin{array}{l} \pm \\
\pm \\
\vdots \\
\vdots \\
\end{array}$ & 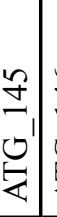 & $\stackrel{0}{\stackrel{4}{4}}$ \\
\hline
\end{tabular}




\begin{tabular}{|c|c|c|c|c|c|c|c|c|c|c|c|c|c|c|c|c|c|c|c|c|c|c|c|c|c|c|c|c|}
\hline$\overline{0}$ & 0 & 0 & 0 & 0 & 0 & 0 & $\sim$ & 6 & 0 & 0 & 0 & $\stackrel{\Xi}{\sim}$ & $\stackrel{\sim}{\sim}$ & $\begin{array}{l}0 \\
\infty\end{array}$ & D & $n$ & $\simeq$ & 0 & 0 & 0 & $\sim$ & $m$ & $\nabla$ & $\sim$ & $m$ & $\sim$ & 0 & 0 \\
\hline$\frac{U}{a}$ & 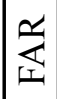 & $\sum_{\text {寽 }}$ & $\sum_{\text {䍃 }}$ & $\begin{array}{l}U \\
\text { IT } \\
\end{array}$ & $\begin{array}{l}u \\
I \\
\end{array}$ & 䍃 & $\frac{\alpha}{4}$ & 䍃 & 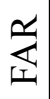 & 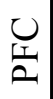 & $\begin{array}{l}0 \\
0 \\
2\end{array}$ & $\mathbf{Z}$ & 㞱 & 妾 & 至 & $\begin{array}{l}u \\
\frac{1}{2} \\
\end{array}$ & $\underset{4}{\frac{2}{4}}$ & $\mid \begin{array}{c}u \\
\frac{1}{L}\end{array}$ & $\begin{array}{c}u \\
\frac{1}{2}\end{array}$ & $\begin{array}{c}U_{I} \\
\frac{T}{2}\end{array}$ & 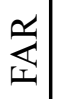 & $\underset{4}{2}$ & $\begin{array}{l}0 \\
\underline{I} \\
\end{array}$ & $\left|\begin{array}{c}U \\
\frac{1}{2} \\
2\end{array}\right|$ & 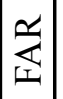 & $\left|\begin{array}{c}0 \\
\frac{1}{2} \\
\end{array}\right|$ & $\begin{array}{l}U_{T} \\
\frac{T}{2}\end{array}$ & $\begin{array}{l}U \\
\frac{T}{2}\end{array}$ \\
\hline$\sum_{\infty} \frac{0}{\sigma}$ & 工 & 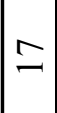 & 그 & $\infty$ & $\infty$ & 二 & 기 & 二 & 工 & $\infty$ & $\infty$ & $\cong$ & $\cong$ & $\simeq$ & $\simeq$ & $\stackrel{\sim}{.}$ & 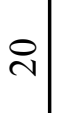 & సે & $\infty$ & 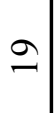 & 구 & I & $\stackrel{\infty}{-}$ & 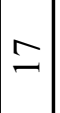 & 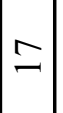 & $\vec{\sim}$ & $\bar{\sim}$ & $\approx$ \\
\hline 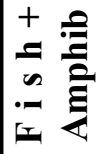 & 0 & 0 & 0 & - & - & 0 & 0 & 0 & 0 & 0 & 0 & 0 & 0 & 0 & 0 & 0 & 0 & 0 & 0 & 0 & 0 & 0 & 0 & 0 & 0 & 0 & 0 & 0 \\
\hline$\frac{\tilde{Z}}{\tilde{z}}$ & 0 & 0 & 0 & - & - & 0 & 0 & 0 & 0 & 0 & 0 & - & -1 & -1 & -1 & -1 & -1 & 0 & -1 & - & 0 & 0 & - & - & 0 & - & - & 0 \\
\hline$\stackrel{2}{0}$ & 0 & 0 & 0 & - & - & 0 & 0 & 0 & 0 & 0 & 0 & 0 & 0 & 0 & 10 & 0 & 0 & 0 & 0 & 0 & 0 & 0 & 0 & 0 & 0 & 0 & 0 & 0 \\
\hline 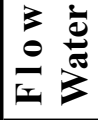 & 0 & 0 & 0 & 0 & 0 & 0 & 0 & 10 & 0 & 0 & 0 & 0 & 0 & 0 & 0 & 0 & 0 & 0 & 0 & 0 & 0 & 0 & 0 & 0 & 0 & 0 & 0 & 0 \\
\hline $\begin{array}{l}\grave{\Xi} \\
\stackrel{\varrho}{\Xi} \\
\Xi\end{array}$ & 0 & 0 & 0 & - & - & 0 & 0 & 10 & 0 & 0 & 0 & - & - & -1 & -1 & -1 & -1 & 0 & - & - & 0 & 0 & - & - & 0 & - & - & 0 \\
\hline $\overrightarrow{0}$ & - & - & - & 0 & 0 & -1 & -1 & - & - & - & - & 0 & 0 & 0 & 0 & 0 & 0 & - & 0 & 0 & - & - & 0 & 0 & -1 & 0 & 0 & - \\
\hline 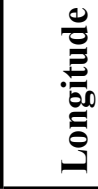 & 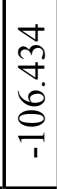 & $\left|\begin{array}{l}\sigma \\
0 \\
+ \\
0 \\
0 \\
\hdashline \\
1\end{array}\right|$ & 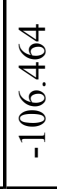 & $\begin{array}{l}\overline{0} \\
+ \\
0 \\
0 \\
1 \\
1\end{array}$ & $\begin{array}{l}\bar{b} \\
+ \\
\dot{0} \\
0 \\
1\end{array}$ & 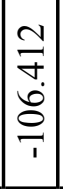 & $\left|\begin{array}{c}0 \\
\infty \\
m \\
\vdots \\
0 \\
1\end{array}\right|$ & $\mid$\begin{tabular}{c}
0 \\
$\infty$ \\
$n$ \\
$\vdots$ \\
0 \\
\hdashline \\
1
\end{tabular} & 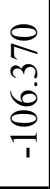 & $\begin{array}{l}\hat{\sigma} \\
n \\
\vdots \\
0 \\
1\end{array}$ & 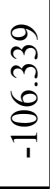 & 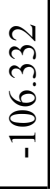 & $\mid$\begin{tabular}{c}
1 \\
$\tilde{n}$ \\
$\tilde{0}$ \\
0 \\
\hdashline \\
1
\end{tabular} & 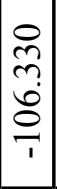 & 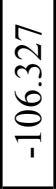 & 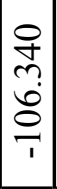 & 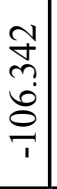 & \begin{tabular}{c}
$\overrightarrow{7}$ \\
$\tilde{n}$ \\
$\vdots$ \\
0 \\
\hdashline \\
1
\end{tabular} & $\begin{array}{c}n \\
\vdots \\
n \\
\vdots \\
\vdots \\
1\end{array}$ & 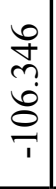 & 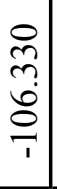 & $\begin{array}{l}m \\
\\
\vdots \\
0 \\
1 \\
1\end{array}$ & $\begin{array}{l}m \\
\\
ٌ \\
0 \\
1\end{array}$ & 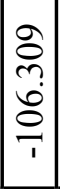 & $\left|\begin{array}{c}0 \\
\infty \\
\\
\vdots \\
0 \\
1\end{array}\right|$ & $\begin{array}{c}1 \\
\infty \\
\sim \\
\vdots \\
\vdots \\
1\end{array}$ & 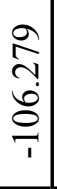 & 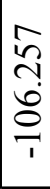 \\
\hline 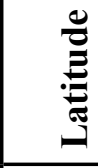 & $\begin{array}{l}\hat{0} \\
0 \\
\dot{y} \\
\gamma\end{array}$ & $\begin{array}{c}\bar{b} \\
0 \\
\dot{y}\end{array}$ & $\begin{array}{l}\hat{b} \\
0 \\
\dot{y}\end{array}$ & $\begin{array}{c}\hat{b} \\
0 \\
\dot{y} \\
\forall\end{array}$ & $\begin{array}{l}\infty \\
0 \\
0 \\
\vdots \\
\ddots \\
\gamma\end{array}$ & 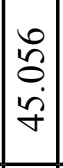 & $\left|\begin{array}{c}\stackrel{9}{ } \\
0 \\
\dot{y} \\
\forall\end{array}\right|$ & $\mid \begin{array}{c}\hat{m} \\
\hat{0} \\
\dot{n} \\
\tilde{\gamma}\end{array}$ & 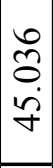 & 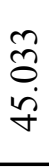 & $\begin{array}{c}\text { तै } \\
0 \\
\dot{n} \\
\forall\end{array}$ & $\begin{array}{c}0 \\
8 \\
\dot{y} \\
\dot{\gamma}\end{array}$ & \begin{tabular}{|c|} 
\\
8 \\
0 \\
$\dot{y}$ \\
$\forall$
\end{tabular} & $\begin{array}{l}\infty \\
\stackrel{2}{2} \\
\dot{f}\end{array}$ & $\begin{array}{l}0 \\
\text { ఫे } \\
\dot{f}\end{array}$ & $\begin{array}{c}\infty \\
0 \\
\dot{y} \\
\forall\end{array}$ & $\begin{array}{l}\bar{D} \\
0 \\
\dot{y}\end{array}$ & $\begin{array}{c}a \\
0 \\
\dot{v} \\
\dot{v}\end{array} \mid$ & $\begin{array}{c}\hat{\widehat{a}} \\
0 \\
\dot{\sigma} \\
\dot{\gamma}\end{array}$ & 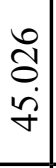 & $\begin{array}{l}0 \\
\mathcal{N} \\
0 \\
\stackrel{f}{f}\end{array}$ & 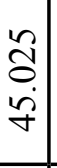 & 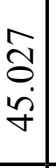 & 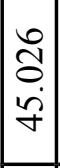 & $\mid \begin{array}{c}\infty \\
0 \\
\dot{y} \\
\gamma\end{array}$ & 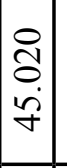 & $\begin{array}{c}a \\
0 \\
\dot{b} \\
\dot{\sigma}\end{array}$ & $\begin{array}{l}\vec{\sigma} \\
0 \\
\dot{y}\end{array}$ \\
\hline 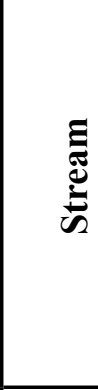 & $\begin{array}{l}0 \\
3 \\
0 \\
0 \\
0 \\
0 \\
0 \\
0 \\
0 \\
0 \\
0\end{array}$ & $\mid \begin{array}{l}0 \\
3 \\
1 \\
0 \\
0 \\
0 \\
0 \\
1 \\
2 \\
0 \\
0\end{array}$ & $\mid \begin{array}{l}0 \\
3 \\
0 \\
0 \\
0 \\
0 \\
0 \\
0 \\
0 \\
0 \\
0\end{array}$ & 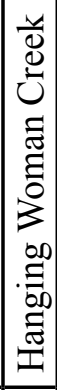 & 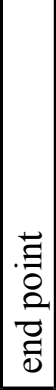 & 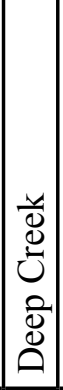 & $\left|\begin{array}{l}0 \\
3 \\
1 \\
0 \\
0 \\
0 \\
\vdots \\
ت \\
2 \\
0 \\
0 \\
0\end{array}\right|$ & 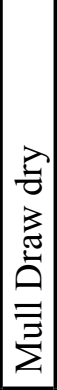 & 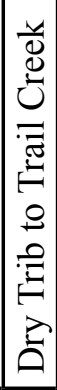 & 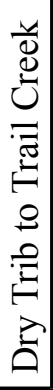 & 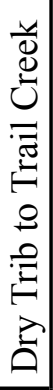 & 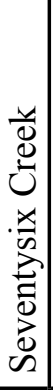 & 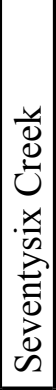 & 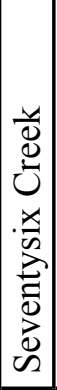 & 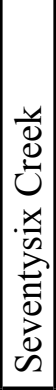 & 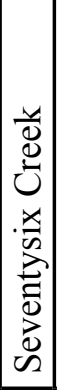 & 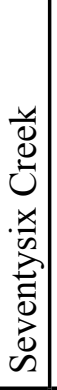 & 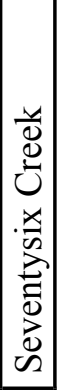 & 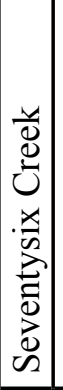 & $\begin{array}{c} \\
\vec{\Xi} \\
. \\
\stackrel{\Xi}{a} \\
\vec{\Xi}\end{array}$ & 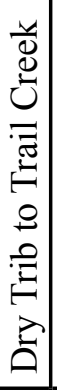 & 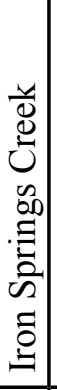 & 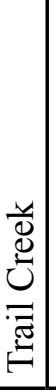 & 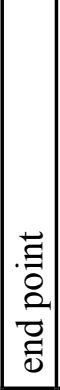 & 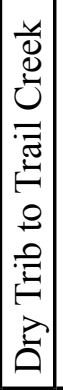 & 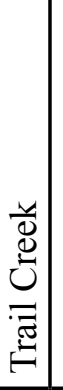 & 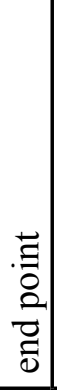 & 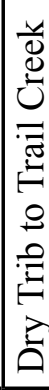 \\
\hline
\end{tabular}

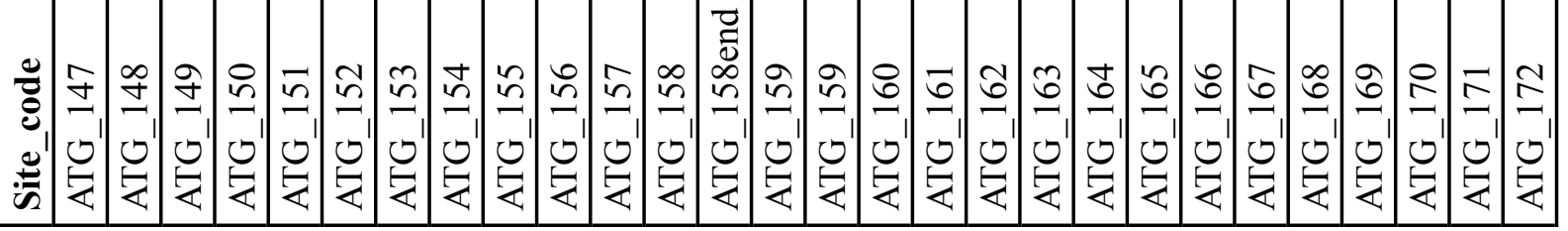




\begin{tabular}{|c|c|c|c|c|c|c|c|c|c|c|c|c|c|c|c|c|c|c|c|c|c|c|c|c|c|c|}
\hline$\overline{\mathrm{u}}$ & 6 & $=$ & 0 & $n$ & 0 & 0 & 0 & 0 & 0 & 0 & $\widehat{\sim}$ & $\ddot{\sim}$ & $\simeq$ & $=$ & $a$ & $\nabla$ & $\infty$ & $\sim$ & $\nabla$ & 9 & $\cong$ & 0 & 0 & 0 & 0 & $r$ \\
\hline$\frac{U}{U}$ & $\sum_{\text {寽 }}$ & 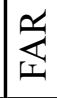 & 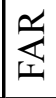 & 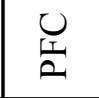 & $\begin{array}{l}u \\
\frac{1}{2} \\
\end{array}$ & 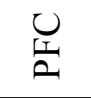 & $\underset{\text { 䍃 }}{2}$ & 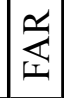 & $\begin{array}{l}0 \\
\text { Yr } \\
2\end{array}$ & $\begin{array}{l}u \\
I \\
\end{array}$ & 䍃 & 䍃 & 齐 & 蛋 & 齐 & $\begin{array}{l}u \\
1 \\
\end{array}$ & 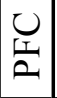 & 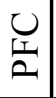 & 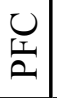 & $\underset{\text { 寽 }}{4}$ & 总 & $\begin{array}{l}u \\
\frac{1}{2}\end{array}$ & $\begin{array}{l}U \\
\frac{T}{\alpha}\end{array}$ & $\begin{array}{c}U \\
\end{array}$ & $\begin{array}{l}U \\
\text { U. } \\
\underline{L}\end{array}$ & $\underset{\text { 乐 }}{|c|}$ \\
\hline$\sum_{\infty} \frac{0}{ \pm}$ & I & 근 & 근 & ปิ & 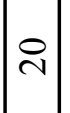 & 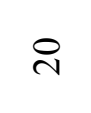 & I & 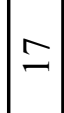 & $\approx$ & $\approx$ & 0 & 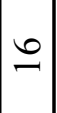 & 二 & 二 & 二 & 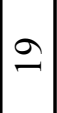 & 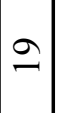 & $\stackrel{\sim}{ }$ & ิ & $\stackrel{6}{-1}$ & $\because$ & $\approx$ & $\curvearrowright$ & $\curvearrowright$ & $\stackrel{2}{2}$ & 二 \\
\hline 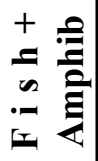 & 0 & 0 & 0 & 0 & 0 & 0 & 0 & 0 & 0 & 0 & 0 & 0 & 0 & 0 & 0 & 0 & 0 & 0 & 0 & 0 & 0 & 0 & 0 & 0 & 0 & - \\
\hline 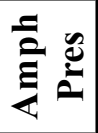 & - & - & 0 & - & - & - & - & - & - & - & - & -1 & 0 & 0 & 0 & -1 & -1 & 0 & 0 & -1 & -1 & - & -1 & 0 & 0 & - \\
\hline$\stackrel{\infty}{\infty}$ & 0 & 0 & 0 & 0 & 0 & 0 & 0 & 0 & 0 & 10 & 0 & 0 & 0 & 0 & 0 & 0 & 0 & 0 & 0 & 0 & 0 & 0 & 0 & 0 & 0 & - \\
\hline 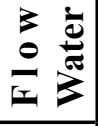 & 0 & 0 & 0 & - & - & - & 0 & 0 & 0 & 10 & 0 & 0 & 0 & 0 & 0 & 0 & 0 & 0 & 0 & 0 & 0 & 0 & - & 0 & 0 & - \\
\hline$\stackrel{\grave{\Xi}}{\stackrel{0}{\Xi}}$ & - & - & 0 & 0 & 0 & 0 & - & - & - & - & -1 & -1 & 0 & 0 & 0 & -1 & -1 & 0 & 0 & -1 & -1 & -1 & 0 & 0 & 0 & 0 \\
\hline $\overrightarrow{0}$ & 0 & 0 & - & 0 & 0 & 0 & 0 & 0 & 0 & 0 & 0 & 0 & -1 & -1 & -1 & 0 & 0 & - & - & 0 & 0 & 0 & 0. & - & - & 0 \\
\hline 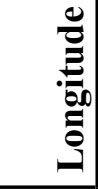 & \begin{tabular}{l}
0 \\
\multirow{2}{2}{} \\
0 \\
0 \\
0 \\
1
\end{tabular} & 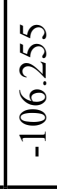 & 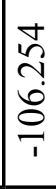 & 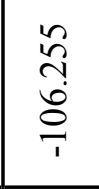 & 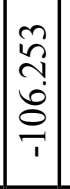 & 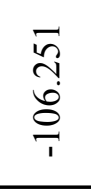 & 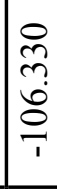 & 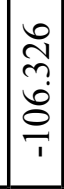 & $\left|\begin{array}{l}8 \\
0 \\
n \\
8 \\
0 \\
\hdashline \\
1\end{array}\right|$ & $\mid \begin{array}{l}n \\
n \\
n \\
0 \\
0 \\
1\end{array}$ & 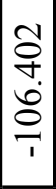 & \begin{tabular}{l}
8 \\
8 \\
\multirow{+}{+}{} \\
$\dot{0}$ \\
$\circ$ \\
1
\end{tabular} & 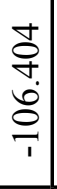 & 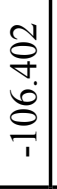 & $\mid$\begin{tabular}{l}
8 \\
0 \\
+ \\
$\dot{0}$ \\
0 \\
\hdashline 1
\end{tabular} & $\mid \begin{array}{l}\hat{a} \\
\tilde{n} \\
0 \\
0 \\
1 \\
1\end{array}$ & 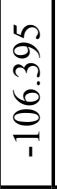 & 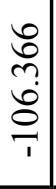 & $\begin{array}{l}n \\
0 \\
n \\
8 \\
0 \\
1\end{array}$ & 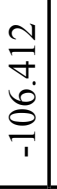 & $\begin{array}{l}= \\
\vec{\nabla} \\
\dot{0} \\
\stackrel{0}{7} \\
1\end{array}$ & 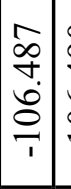 & 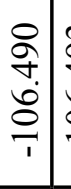 & $\begin{array}{l}\tilde{\alpha} \\
\dot{\sigma} \\
\stackrel{0}{0} \\
\frac{1}{1}\end{array}$ & $\begin{array}{l}\Omega \\
0 \\
n \\
0 \\
0 \\
1 \\
1\end{array}$ & \begin{tabular}{l}
$n$ \\
$\tilde{\sigma}$ \\
$\stackrel{0}{0}$ \\
\hdashline \\
\hdashline
\end{tabular} \\
\hline 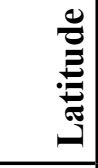 & $\begin{array}{l}\bar{\sigma} \\
\dot{y} \\
\dot{\sigma}\end{array}$ & $\begin{array}{l}\bar{\sigma} \\
0 \\
\dot{y}\end{array}$ & $\begin{array}{l} \pm \\
0 \\
0 \\
\dot{\sigma}\end{array}$ & $\begin{array}{l}\stackrel{\nabla}{0} \\
\dot{0} \\
\ddot{\sigma}\end{array}$ & $\begin{array}{c}n \\
0 \\
i \\
f \\
\end{array}$ & $\begin{array}{l}0 \\
0 \\
0 \\
\dot{\gamma}\end{array}$ & 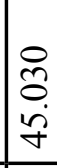 & 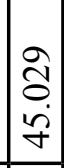 & $\begin{array}{l}n \\
\tilde{0} \\
\tilde{y} \\
\gamma \\
\end{array}$ & $\begin{array}{l}n \\
\tilde{n} \\
n \\
\tilde{\gamma} \\
\end{array}$ & $\begin{array}{c}a \\
\dot{0} \\
\dot{y} \\
\dot{y}\end{array}$ & $\begin{array}{c}a \\
\dot{0} \\
\dot{b} \\
\dot{y}\end{array}$ & \begin{tabular}{|c}
0 \\
0 \\
0 \\
$\dot{y}$ \\
\end{tabular} & \begin{tabular}{l}
\multirow{2}{\delta}{} \\
0 \\
$\dot{y}$ \\
\end{tabular} & $\mid \begin{array}{c}0 \\
\hat{0} \\
\dot{y} \\
\end{array}$ & 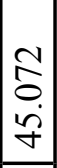 & \begin{tabular}{|c|}
$\overline{\widehat{O}}$ \\
0 \\
$\dot{y}$ \\
$\dot{\sigma}$
\end{tabular} & $\begin{array}{l}0 \\
0 \\
\dot{0} \\
\dot{y}\end{array}$ & $\begin{array}{l}0 \\
0 \\
\dot{y} \\
\dot{+}\end{array}$ & \begin{tabular}{l}
\multirow{0}{*}{} \\
0 \\
$\dot{f}$ \\
$\dot{+}$
\end{tabular} & $\begin{array}{c}3 \\
0 \\
0 \\
i \\
\dot{\gamma}\end{array}$ & 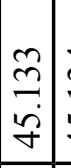 & 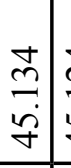 & 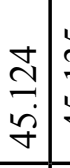 & $\begin{array}{l}n \\
\tilde{n} \\
\dot{f} \\
\dot{\sigma}\end{array}$ & $\begin{array}{l}\hat{S} \\
0 \\
\dot{y}\end{array}$ \\
\hline 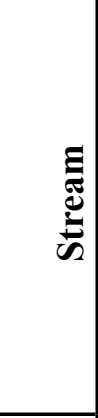 & 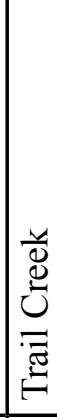 & 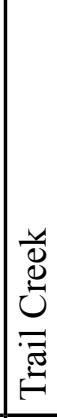 & 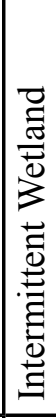 & 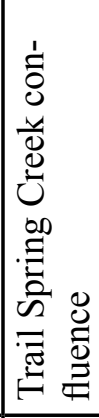 & 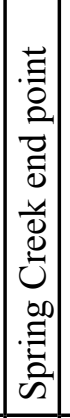 & 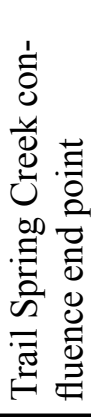 & 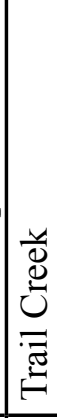 & 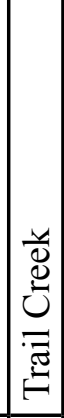 & 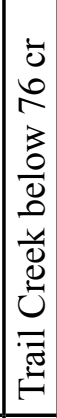 & 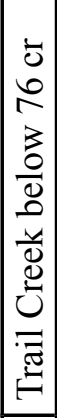 & 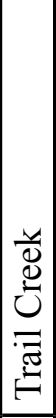 & 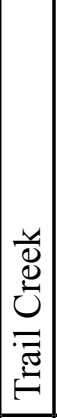 & 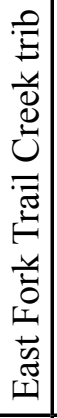 & 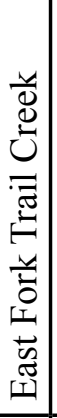 & 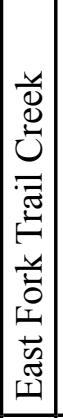 & 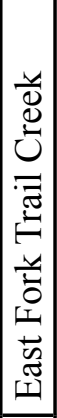 & 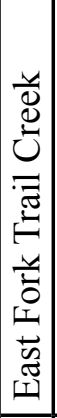 & 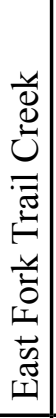 & 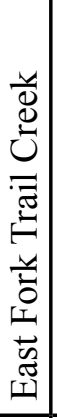 & 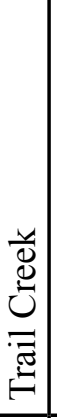 & 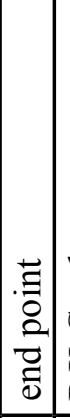 & 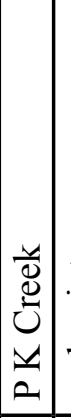 & $\begin{array}{c} \\
\vec{\Xi} \\
\vdots \\
\bar{z} \\
\overrightarrow{0}\end{array}$ & 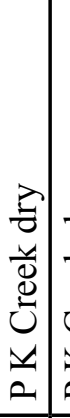 & 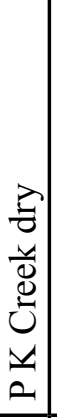 & 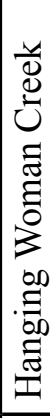 \\
\hline 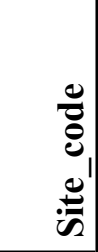 & 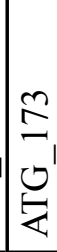 & 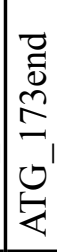 & 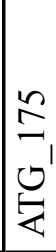 & 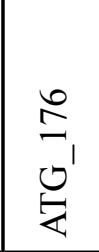 & 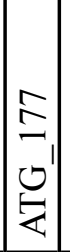 & $\begin{array}{l}\stackrel{\infty}{I} \\
\underset{0}{\mid} \\
\stackrel{4}{4}\end{array}$ & $\begin{array}{l}9 \\
2 \\
0 \\
0 \\
2\end{array}$ & $\mid \begin{array}{l}8 \\
\infty \\
0 \\
0 \\
\\
\end{array}$ & \begin{tabular}{l}
$\infty$ \\
$\infty$ \\
0 \\
\multirow{2}{*}{}
\end{tabular} & $\left|\begin{array}{l}\infty \\
\infty \\
0 \\
0 \\
E \\
\gtrless\end{array}\right|$ & $\left|\begin{array}{l}\infty \\
\infty \\
0 \\
0 \\
6\end{array}\right|$ & \begin{tabular}{l|}
+ \\
$\infty$ \\
$\vdots$ \\
$\vdots$ \\
6 \\
\end{tabular} & 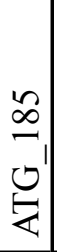 & $\begin{array}{l}\circ \\
\infty \\
-1 \\
0 \\
\end{array}$ & $\left|\begin{array}{c}0 \\
0 \\
0 \\
0 \\
\\
\end{array}\right|$ & \begin{tabular}{|l|}
$\infty$ \\
$\infty$ \\
0 \\
0 \\
$⿱ ⺊$ \\
$<$
\end{tabular} \mid & $\begin{array}{l}2 \\
\infty \\
0 \\
\qquad \\
\end{array}$ & 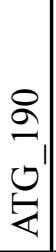 & 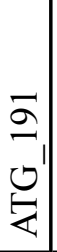 & 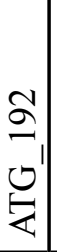 & 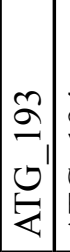 & 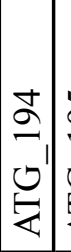 & \begin{tabular}{l}
$n$ \\
$\approx$ \\
0 \\
\multirow{2}{*}{}
\end{tabular} & 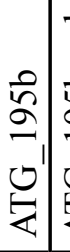 & 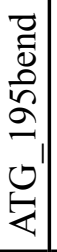 & \begin{tabular}{l}
0 \\
2 \\
0 \\
\multirow{2}{*}{}
\end{tabular} \\
\hline
\end{tabular}




\begin{tabular}{|c|c|c|c|c|c|c|c|c|c|c|c|c|c|c|c|c|c|c|c|c|c|c|c|c|c|c|c|c|}
\hline$\overline{\tilde{u}}$ & $r$ & $\infty$ & $\because$ & $\infty$ & 0 & 10 & 0 & 0 & 0 & 0 & $\simeq$ & $\infty$ & $\infty$ & 0 & 0 & $\infty$ & $n$ & $\curvearrowright$ & 0 & 0 & 0 & 0 & 0 & 0 & 0 & 0 & 0 & 0 \\
\hline$\frac{U}{a}$ & 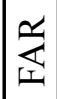 & $\begin{array}{l}0 \\
\frac{1}{2} \\
2\end{array}$ & 运 & 至 & \begin{tabular}{|l}
0 \\
$\frac{1}{2}$
\end{tabular} & $\frac{U}{\mathrm{~L}}$ & $\frac{U}{L}$ & $\begin{array}{l}u \\
\frac{I}{2} \\
\end{array}$ & $\begin{array}{l}0 \\
\frac{I}{2}\end{array}$ & U & $\underset{\text { 空 }}{2}$ & $\begin{array}{l}u \\
\frac{I}{2} \\
\end{array}$ & 岱 & $\underset{\text { 寽 }}{2}$ & 䍃 & 䍃 & $\underset{4}{\frac{2}{4}}$ & $\underset{4}{\stackrel{2}{4}}$ & 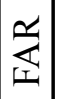 & $\underset{2}{\frac{\alpha}{4}}$ & $\underset{\text { 寽 }}{2}$ & $\begin{array}{l}U \\
\frac{I}{2} \\
\end{array}$ & $\begin{array}{l}U \\
\frac{I}{2} \\
\end{array}$ & 选 & $\left|\begin{array}{l}0 \\
\frac{1}{2} \\
2\end{array}\right|$ & 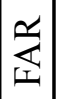 & 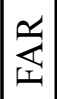 & 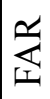 \\
\hline$\sum_{\infty} \frac{0}{\Xi}$ & 工 & 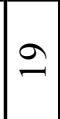 & 0 & $\simeq$ & $\infty$ & $\infty$ & 尺े & 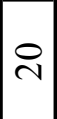 & 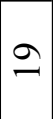 & 9 & $\because$ & ํ. & ㄱ & 0 & 0 & 二 & 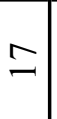 & 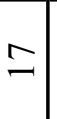 & 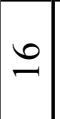 & 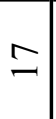 & 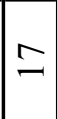 & $\infty$ & $\infty$ & 0 & $\infty$ & $\Xi$ & \pm & $\Xi$ \\
\hline 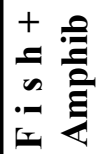 & - & 0 & 0 & 0 & 0 & 0 & - & - & - & - & 0 & - & - & 0 & 0 & 0 & 0 & 0 & 0 & 0 & 10 & 0 & 0 & 0 & 0 & 0 & 0 & 0 \\
\hline 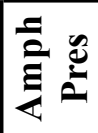 & - & - & - & 0 & 0 & 0 & - & - & - & - & 0 & - & - & - & - & 0 & 0 & 0 & 0 & 0 & 0 & 0 & - & - & 10 & 0 & 0 & 0 \\
\hline$\stackrel{2}{0}$ & - & 0 & 0 & 0 & 0 & 10 & - & - & - & - & 0 & - & - & 0 & 0 & 0 & 0 & 0 & 0 & 0 & 0 & 0 & 0 & 0 & 0 & 0 & 0 & 0 \\
\hline 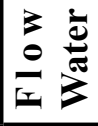 & - & 0 & 0 & 0 & 0 & 10 & 0 & 0 & - & - & 0 & -1 & - & - & -1 & 0 & 0 & 0 & 0 & 0 & 0 & 0 & 0 & 0 & 0 & 0 & 0 & 0 \\
\hline $\begin{array}{l}\grave{\Xi} \\
\stackrel{0}{\Xi}\end{array}$ & 0 & - & - & 0 & 0 & 10 & - & - & 0 & 0 & 0 & 0 & 0 & 0 & 0 & 10 & 0 & 0 & 0 & 0 & 0 & 0 & - & - & 0 & 0 & 0 & 0 \\
\hline$\vec{b}$ & 0 & 0 & 0 & -1 & - & - & 0 & 0 & 0 & 0 & - & 0 & 0 & 0 & 0 & - & -1 & -1 & -1 & - & - & - & 0 & 0 & - & -1 & - & - \\
\hline 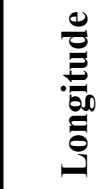 & $\begin{array}{l}n \\
\tilde{\sigma} \\
\stackrel{5}{0} \\
\stackrel{0}{0} \\
1\end{array}$ & \begin{tabular}{l}
$n$ \\
$b$ \\
+ \\
$\dot{0}$ \\
\hdashline \\
1
\end{tabular} & $\left|\begin{array}{l}-1 \\
0 \\
+ \\
0 \\
0 \\
1 \\
1\end{array}\right|$ & $\left|\begin{array}{l}\overrightarrow{0} \\
+ \\
\dot{0} \\
0 \\
\hdashline \\
1\end{array}\right|$ & $\mid$\begin{tabular}{l}
$\tilde{n}$ \\
\multirow{2}{*}{} \\
$\dot{0}$ \\
0 \\
1
\end{tabular} & $\begin{array}{l}\hat{\gamma} \\
\dot{\gamma} \\
\dot{0} \\
0 \\
\end{array}$ & $\mid$\begin{tabular}{l}
+ \\
$\infty$ \\
\multirow{+}{*}{} \\
$\stackrel{0}{0}$ \\
0 \\
1
\end{tabular} & 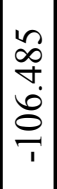 & 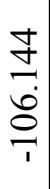 & $\begin{array}{l}\stackrel{\infty}{+} \\
\stackrel{0}{0} \\
\frac{1}{1}\end{array}$ & 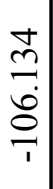 & $\left|\begin{array}{l}0 \\
6 \\
0 \\
0 \\
0 \\
1\end{array}\right|$ & $\begin{array}{l}0 \\
\stackrel{0}{0} \\
\stackrel{0}{1} \\
\end{array}$ & $\mid \begin{array}{l}\infty \\
0 \\
-1 \\
0 \\
0 \\
1\end{array}$ & $\left|\begin{array}{c}8 \\
0 \\
\dot{8} \\
0 \\
1\end{array}\right|$ & 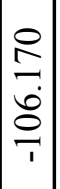 & $\begin{array}{c}\vec{\infty} \\
-1 \\
0 \\
0 \\
1\end{array}$ & \begin{tabular}{c}
$\infty$ \\
$\infty$ \\
-1 \\
0 \\
\hdashline \\
1
\end{tabular} & 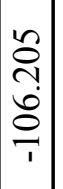 & \begin{tabular}{l}
\multirow{2}{*}{} \\
$\stackrel{0}{0}$ \\
$\stackrel{1}{1}$
\end{tabular} & $\begin{array}{l}\vec{n} \\
\stackrel{0}{0} \\
0 \\
1\end{array}$ & \begin{tabular}{l}
0 \\
0 \\
\hdashline \\
0 \\
0 \\
1
\end{tabular} & $\begin{array}{l}\stackrel{+}{ \pm} \\
\stackrel{0}{0} \\
\stackrel{0}{1}\end{array}$ & 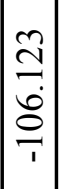 & $\left|\begin{array}{c}\hat{\imath} \\
\hdashline \\
0 \\
\hdashline \\
1\end{array}\right|$ & 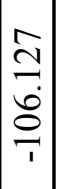 & 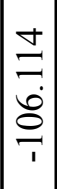 & $\begin{array}{l}\infty \\
0 \\
0 \\
0 \\
0 \\
1\end{array}$ \\
\hline 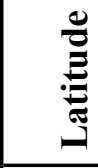 & 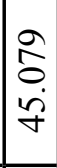 & $\begin{array}{l}\infty \\
\infty \\
0 \\
\dot{f} \\
\forall\end{array}$ & 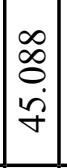 & $\left|\begin{array}{l}\hat{\infty} \\
0 \\
0 \\
\dot{\gamma}\end{array}\right|$ & 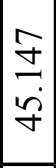 & $\frac{n}{\stackrel{f}{f}}$ & $\begin{array}{l}\stackrel{+}{m} \\
\stackrel{7}{\sigma} \\
\forall\end{array}$ & $\mid$\begin{tabular}{c}
0 \\
$m$ \\
\hdashline \\
$\stackrel{n}{\sigma}$ \\
\end{tabular} & 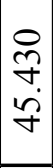 & 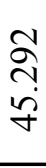 & $\begin{array}{l}\stackrel{+}{m} \\
\dot{f} \\
\dot{\sigma}\end{array}$ & 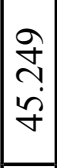 & 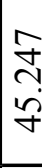 & 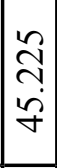 & 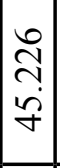 & 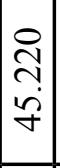 & 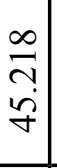 & 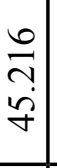 & 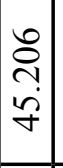 & $\begin{array}{c}\vec{J} \\
\stackrel{-}{\dot{f}} \\
\dot{f}\end{array}$ & $\begin{array}{l}g \\
\stackrel{g}{+} \\
\dot{f}\end{array}$ & 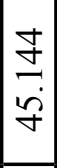 & 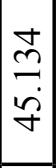 & 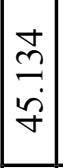 & 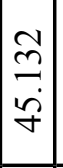 & \begin{tabular}{c}
$\stackrel{0}{1}$ \\
\hdashline \\
$\dot{\gamma}$
\end{tabular} & $\begin{array}{c}\hat{a} \\
8 \\
\dot{y} \\
y\end{array}$ & $\begin{array}{l}\hat{\hat{O}} \\
0 \\
\dot{y} \\
\gamma\end{array}$ \\
\hline & 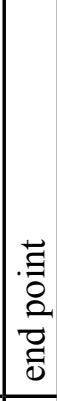 & 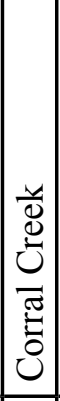 & 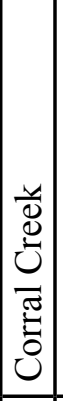 & 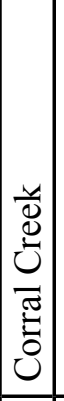 & 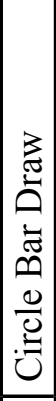 & 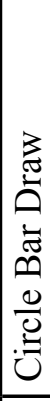 & 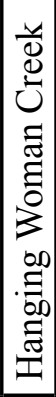 & 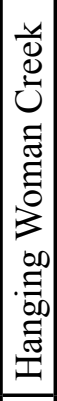 & 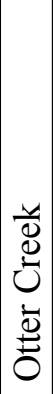 & 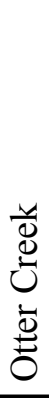 & 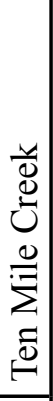 & 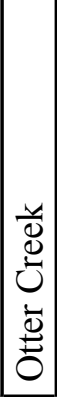 & 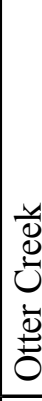 & 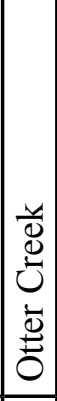 & 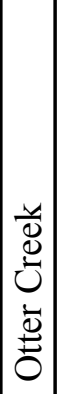 & 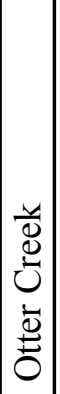 & 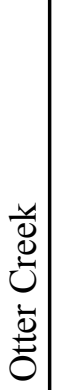 & 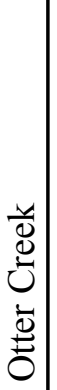 & 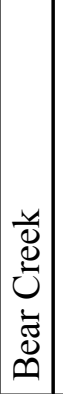 & 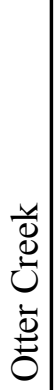 & 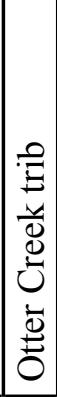 & 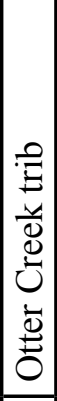 & 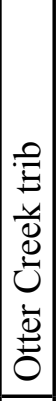 & 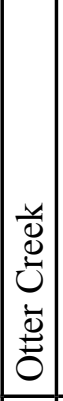 & 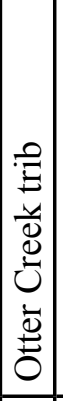 & 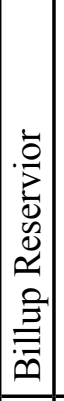 & 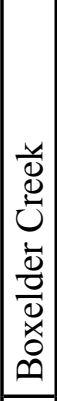 & 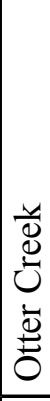 \\
\hline
\end{tabular}

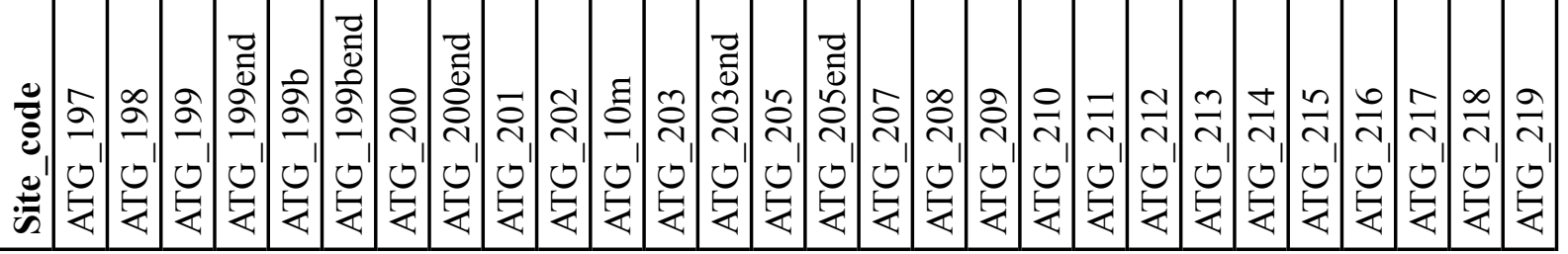




\begin{tabular}{|c|c|c|c|c|c|c|c|c|c|c|c|c|c|c|c|c|c|c|c|c|c|c|c|}
\hline $\bar{v}$ & 0 & $\infty$ & $\sim$ & $\nabla$ & $n$ & $\sim$ & $\sim \mid$ & 0 & $\nabla$ & $\overparen{\sim}$ & $\approx$ & 2 & $\approx$ & 6 & in & $\sim$ & 0 & 0 & 0 & $N \mid$ & $\sim \mid$ & 0 & 0 \\
\hline$\frac{U}{a}$ & 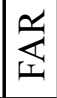 & 䍃 & 足 & 䍃 & 䍃 & 䍃 & 䍃 & 齐 & $\underset{4}{\stackrel{2}{4}}$ & $\mathbf{Z}$ & 齐 & $\underset{\text { 紊 }}{2}$ & 䍃 & 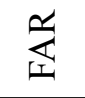 & $\begin{array}{l}u \\
\underline{T} \\
\end{array}$ & 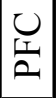 & $\begin{array}{l}U_{1} \\
\frac{1}{2}\end{array}$ & $\begin{array}{l}U_{\mathrm{T}} \\
\underline{\underline{L}}\end{array}$ & 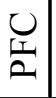 & $\begin{array}{c}0 \\
\mathrm{~L} \\
2\end{array}$ & $\begin{array}{l}u \\
T \\
\end{array}$ & $\begin{array}{l}0 \\
\mathrm{I} \\
\mathrm{L}\end{array}$ & $\begin{array}{l}0 \\
\mathbf{L} \\
\mathbf{L}\end{array}$ \\
\hline$\sum_{\Delta} \frac{0}{\Xi}$ & 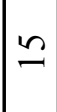 & $\underset{-1}{0}$ & $\stackrel{\infty}{-}$ & 工 & I & \pm & \pm & \pm & \pm & $\simeq$ & $\cong$ & $\simeq$ & $\cong$ & $\Xi$ & $\stackrel{\curvearrowright}{~}$ & $\approx$ & $\infty$ & 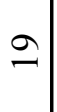 & $\stackrel{\sim}{ }$ & 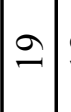 & $\infty$ & 9 & 9 \\
\hline 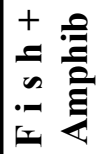 & 0 & 0 & 0 & 0 & 0 & 0 & 0 & 0 & 0 & 0 & 0 & 0 & 0 & 0 & 0 & -1 & -1 & -1 & 0 & 0 & 0. & -1 & - \\
\hline 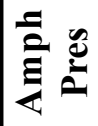 & - & - & - & - & - & - & -1 & 0 & 0 & -1 & 0 & -1 & 0 & 0 & - & - & -1 & - & -1 & 0 & 0 & -1 & - \\
\hline$\stackrel{0}{\infty}$ & 0 & 0 & 0 & 0 & 0 & 0 & 0 & 0 & 0 & 0 & 0 & 0 & 0 & 0 & 0 & -1 & 0 & 0 & 0 & 10 & 0 & -1 & - \\
\hline 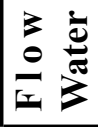 & 0 & 0 & 0 & 0 & 0 & 0 & 0 & 0 & 0 & 0 & 0 & 0 & 0 & 0 & 0 & -1 & -1 & -1 & 0 & 0 & 0 & -1 & - \\
\hline$\stackrel{\grave{\Xi}}{\stackrel{\Xi}{\Xi}}$ & - & - & - & - & -7 & -1 & -1 & 0 & 0 & -1 & 0 & -1 & 0 & 0 & - & 0 & 0 & 0 & 0 & 0 & 0 & 0 & 0 \\
\hline$\vec{D}$ & 0 & 0 & 0 & 0 & 0 & 0 & 0 & -1 & -1 & 0 & -1 & 0 & - & - & 0 & 0 & 0 & 0 & 0 & -1 & -1 & 0 & 0 \\
\hline 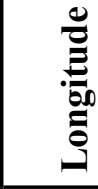 & $\begin{array}{l}2 \\
8 \\
\dot{0} \\
\circ \\
1 \\
1\end{array}$ & $\mid \begin{array}{l}\infty \\
8 \\
0 \\
\dot{0} \\
0 \\
1\end{array}$ & $\mid \begin{array}{l}2 \\
\delta \\
0 \\
0 \\
1 \\
1\end{array}$ & $\mid \begin{array}{l}2 \\
8 \\
0 \\
0 \\
1 \\
1\end{array}$ & 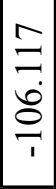 & 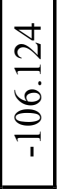 & 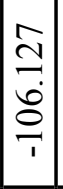 & $\left|\begin{array}{c}0 \\
0 \\
0 \\
\dot{0} \\
\hdashline \\
1\end{array}\right|$ & $\begin{array}{l}\infty \\
0 \\
0 \\
\dot{0} \\
0 \\
1\end{array}$ & \begin{tabular}{l}
$\infty$ \\
\multirow{0}{0}{} \\
$\dot{0}$ \\
0 \\
1
\end{tabular} & 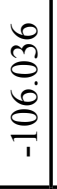 & 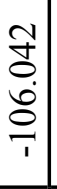 & $\left|\begin{array}{c}0 \\
0 \\
0 \\
\dot{0} \\
0 \\
1\end{array}\right|$ & $\begin{array}{l}\hat{n} \\
0 \\
\stackrel{0}{0} \\
\frac{1}{1}\end{array}$ & $\mid \begin{array}{l}2 \\
2 \\
2 \\
0 \\
1 \\
1\end{array}$ & $\begin{array}{l}0 \\
0 \\
0 \\
0 \\
0 \\
1 \\
1\end{array}$ & \begin{tabular}{c}
\multirow{2}{0}{} \\
0 \\
$\dot{0}$ \\
0 \\
\hdashline \\
1
\end{tabular} & $\begin{array}{l}n \\
0 \\
0 \\
0 \\
1 \\
1\end{array}$ & \begin{tabular}{l}
$\infty$ \\
0 \\
\hdashline \\
$\circ$ \\
0 \\
1
\end{tabular} & $\left|\begin{array}{c}8 \\
\multirow{7}{*}{} \\
0 \\
0 \\
1 \\
1\end{array}\right|$ & 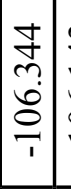 & $\begin{array}{l}\stackrel{9}{\Xi} \\
\stackrel{0}{\circ} \\
\stackrel{1}{1}\end{array}$ & $\begin{array}{l}\stackrel{m}{ \pm} \\
\stackrel{0}{0} \\
\frac{0}{1}\end{array}$ \\
\hline 苞 & $\begin{array}{l}\tilde{y} \\
\infty \\
0 \\
\tilde{\sigma} \\
\tilde{\sigma}\end{array}$ & $\begin{array}{c}0 \\
\infty \\
0 \\
\dot{\gamma} \\
\forall\end{array}$ & $\begin{array}{l}+ \\
\infty \\
0 \\
\dot{\sigma}\end{array}$ & $\mid \begin{array}{l}n \\
\infty \\
0 \\
\dot{\gamma} \\
\forall\end{array}$ & 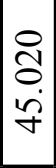 & 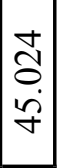 & 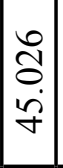 & $\left|\begin{array}{c}2 \\
0 \\
0 \\
y\end{array}\right|$ & \begin{tabular}{|c|}
8 \\
8 \\
$\dot{y}$ \\
$\forall$
\end{tabular} & \begin{tabular}{c}
8 \\
\hdashline \\
$\dot{y}$ \\
+
\end{tabular} & $\begin{array}{l}2 \\
\dot{\partial} \\
\dot{y} \\
\dot{f}\end{array}$ & 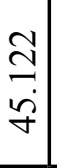 & \begin{tabular}{c}
0 \\
\hdashline \\
$\dot{n}$ \\
$\dot{n}$
\end{tabular} & $\frac{a}{a}$ & 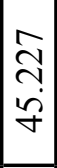 & $\begin{array}{c}\hat{n} \\
\hat{n} \\
\tilde{\sigma} \\
\sigma\end{array}$ & $\begin{array}{c}\hat{n} \\
\hat{y} \\
\tilde{\sigma}\end{array}$ & 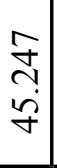 & 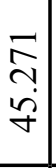 & 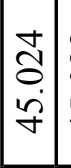 & 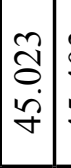 & $\begin{array}{c}0 \\
o \\
\dot{\gamma} \\
\dot{\gamma} \\
\gamma\end{array}$ & $\begin{array}{l}\qquad \\
\delta \\
+ \\
\dot{f}\end{array}$ \\
\hline 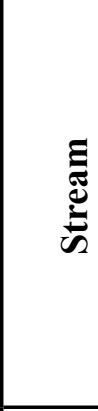 & 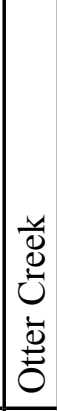 & 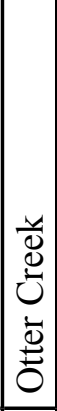 & 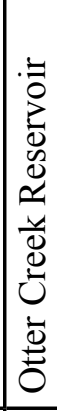 & 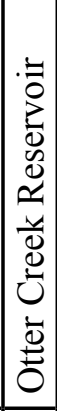 & 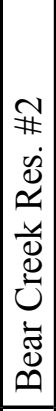 & 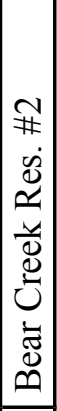 & 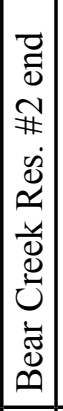 & 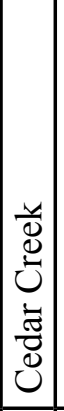 & 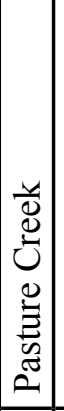 & 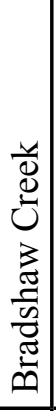 & 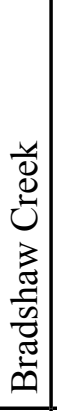 & $\begin{array}{c} \\
\\
00 \\
0 \\
0 \\
0 \\
\infty \\
\vdots \\
\overline{0} \\
0 \\
0 \\
0 \\
\end{array}$ & 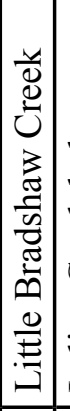 & 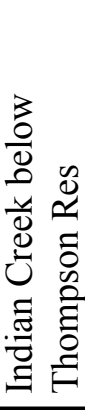 & 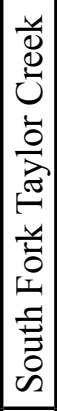 & 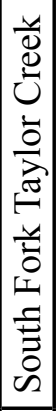 & 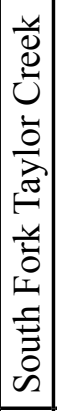 & 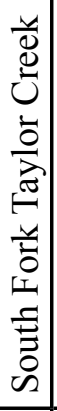 & 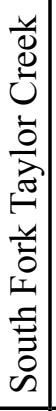 & $\mid \begin{array}{l}3 \\
i \\
0 \\
0 \\
0 \\
0 \\
0 \\
0 \\
3\end{array}$ & \begin{tabular}{|c|}
3 \\
0 \\
0 \\
0 \\
0 \\
0 \\
0 \\
0 \\
0 \\
3
\end{tabular} & 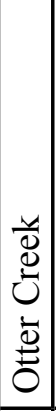 & 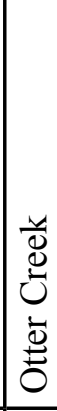 \\
\hline 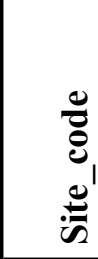 & 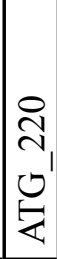 & $\mid \begin{array}{l}\vec{N} \\
\widetilde{N} \\
0 \\
\mathbb{Z}\end{array}$ & 焉 & 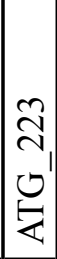 & 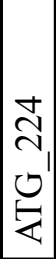 & 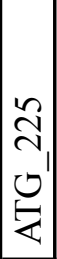 & 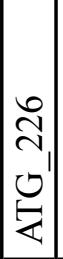 & 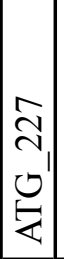 & 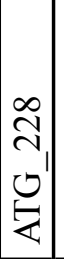 & 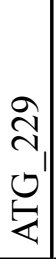 & 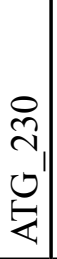 & $\begin{array}{l}\vec{n} \\
\tilde{v}_{1} \\
\overrightarrow{4}\end{array}$ & 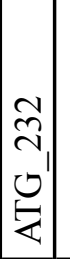 & $\begin{array}{l}\hat{\vartheta}_{1} \\
\bigcup_{1} \\
\text { 岁 }\end{array}$ & 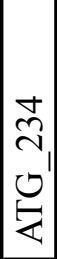 & 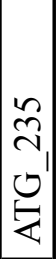 & $\begin{array}{l}0 \\
\infty \\
2 \\
0 \\
\qquad \\
\end{array}$ & 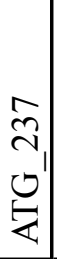 & 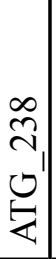 & \begin{tabular}{|c|} 
\\
0 \\
3 \\
0 \\
2 \\
0 \\
0 \\
6 \\
\end{tabular} & $\mid \begin{array}{c}0 \\
0 \\
0 \\
0 \\
3 \\
0 \\
2 \\
0 \\
0 \\
6 \\
\end{array}$ & 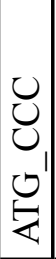 & $\begin{array}{l}0 \\
0 \\
0 \\
0 \\
\text { 安 }\end{array}$ \\
\hline
\end{tabular}





\section{Appendix B. Macroinvertebrate Taxa, Abundance and Metrics for the 20 Collection Sites}





\begin{tabular}{|c|c|c|c|c|c|}
\hline StationID & Site_Name & $\begin{array}{l}\text { Start } \\
\text { Date }\end{array}$ & Activity & Taxon & Abund \\
\hline ATG_124 & Waddle Creek OW Ranch \#1 & $7 / 9 / 2010$ & EMAP_R500 & Chironomus & 13 \\
\hline ATG_124 & Waddle Creek OW Ranch \#1 & $7 / 9 / 2010$ & EMAP_R500 & Berosus & 16 \\
\hline ATG_124 & Waddle Creek OW Ranch \#1 & $7 / 9 / 2010$ & EMAP_R500 & Cricotopus & 123 \\
\hline ATG_124 & Waddle Creek OW Ranch \#1 & $7 / 9 / 2010$ & EMAP_R500 & pseudodiamesa & 2 \\
\hline ATG_124 & Waddle Creek OW Ranch \#1 & $7 / 9 / 2010$ & EMAP_R500 & Corixidae & 135 \\
\hline ATG_124 & Waddle Creek OW Ranch \#1 & $7 / 9 / 2010$ & EMAP_R500 & Paratanytarsus & 45 \\
\hline ATG_124 & Waddle Creek OW Ranch \#1 & $7 / 9 / 2010$ & EMAP_R500 & Helophorus & 2 \\
\hline ATG_124 & Waddle Creek OW Ranch \#1 & $7 / 9 / 2010$ & EMAP_R500 & Tubificidae & 4 \\
\hline ATG_124 & Waddle Creek OW Ranch \#1 & $7 / 9 / 2010$ & EMAP_R500 & Dasyhelea & 6 \\
\hline ATG_124 & Waddle Creek OW Ranch \#1 & $7 / 9 / 2010$ & EMAP_R500 & Enallagma & 4 \\
\hline ATG_124 & Waddle Creek OW Ranch \#1 & $7 / 9 / 2010$ & EMAP_R500 & Ephydra & 8 \\
\hline ATG_124 & Waddle Creek OW Ranch \#1 & $7 / 9 / 2010$ & EMAP_R500 & Notonectidae & 2 \\
\hline ATG_124 & Waddle Creek OW Ranch \#1 & $7 / 9 / 2010$ & EMAP_R500 & Radotanypus & 35 \\
\hline ATG_124 & Waddle Creek OW Ranch \#1 & $7 / 9 / 2010$ & EMAP_R500 & Illybius & 2 \\
\hline ATG_124 & Waddle Creek OW Ranch \#1 & $7 / 9 / 2010$ & EMAP_R500 & Calaparyphus & 1 \\
\hline ATG_124 & Waddle Creek OW Ranch \#1 & $7 / 9 / 2010$ & EMAP_R500 & Agabus & 2 \\
\hline ATG_124 & Waddle Creek OW Ranch \#1 & $7 / 9 / 2010$ & EMAP_R500 & Enallagma annexum & 2 \\
\hline ATG_124 & Waddle Creek OW Ranch \#1 & $7 / 9 / 2010$ & EMAP_R500 & Callibaetis & 14 \\
\hline ATG_124 & Waddle Creek OW Ranch \#1 & $7 / 9 / 2010$ & EMAP_R500 & Physella acuta & 26 \\
\hline ATG_124 & Waddle Creek OW Ranch \#1 & $7 / 9 / 2010$ & EMAP_R500 & Lestes & 21 \\
\hline ATG_124 & Waddle Creek OW Ranch \#1 & $7 / 9 / 2010$ & EMAP_R500 & Orthocladiinae & 26 \\
\hline ATG_124 & Waddle Creek OW Ranch \#1 & $7 / 9 / 2010$ & EMAP_R500 & Hydrobius & 2 \\
\hline ATG_130 & $\begin{array}{l}\text { Hanging Woman Creek uppermost } \\
\text { site in WY private }\end{array}$ & $7 / 9 / 2010$ & EMAP_R500 & Lestes & 4 \\
\hline ATG_130 & $\begin{array}{l}\text { Hanging Woman Creek uppermost } \\
\text { site in WY private }\end{array}$ & $7 / 9 / 2010$ & EMAP_R500 & Tabanus & 28 \\
\hline ATG_130 & $\begin{array}{l}\text { Hanging Woman Creek uppermost } \\
\text { site in WY private }\end{array}$ & $7 / 9 / 2010$ & EMAP_R500 & Corixidae & 400 \\
\hline ATG_130 & $\begin{array}{l}\text { Hanging Woman Creek uppermost } \\
\text { site in WY private }\end{array}$ & 7/9/2010 & EMAP_R500 & Berosus & 2 \\
\hline ATG_130 & $\begin{array}{l}\text { Hanging Woman Creek uppermost } \\
\text { site in WY private }\end{array}$ & $7 / 9 / 2010$ & EMAP_R500 & Laccophilus & 2 \\
\hline ATG_130 & $\begin{array}{l}\text { Hanging Woman Creek uppermost } \\
\text { site in WY private }\end{array}$ & $7 / 9 / 2010$ & EMAP_R500 & Ephydra & 3 \\
\hline ATG_130 & $\begin{array}{l}\text { Hanging Woman Creek uppermost } \\
\text { site in WY private }\end{array}$ & 7/9/2010 & EMAP_R500 & Ablabesmyia & 11 \\
\hline ATG_130 & $\begin{array}{l}\text { Hanging Woman Creek uppermost } \\
\text { site in WY private }\end{array}$ & $7 / 9 / 2010$ & EMAP_R500 & Oreodytes & 2 \\
\hline
\end{tabular}




\begin{tabular}{|c|c|c|c|c|c|}
\hline StationID & Site_Name & $\begin{array}{l}\text { Start } \\
\text { Date }\end{array}$ & Activity & Taxon & Abund \\
\hline ATG_130 & $\begin{array}{l}\text { Hanging Woman Creek uppermost } \\
\text { site in WY private }\end{array}$ & 7/9/2010 & EMAP_R500 & Enochrus & 3 \\
\hline ATG_130 & $\begin{array}{l}\text { Hanging Woman Creek uppermost } \\
\text { site in WY private }\end{array}$ & 7/9/2010 & EMAP_R500 & Helophorus & 4 \\
\hline ATG_130 & $\begin{array}{l}\text { Hanging Woman Creek uppermost } \\
\text { site in WY private }\end{array}$ & 7/9/2010 & EMAP_R500 & Chironominae & 37 \\
\hline ATG_130 & $\begin{array}{l}\text { Hanging Woman Creek uppermost } \\
\text { site in WY private }\end{array}$ & 7/9/2010 & EMAP_R500 & Enallagma & 7 \\
\hline ATG_132 & $\begin{array}{l}\text { Hanging Woman Creek county road } \\
\text { crossing OW Ranch \#2 }\end{array}$ & 7/9/2010 & EMAP_R500 & Agabus & 1 \\
\hline ATG_132 & $\begin{array}{l}\text { Hanging Woman Creek county road } \\
\text { crossing OW Ranch \#2 }\end{array}$ & 7/9/2010 & EMAP_R500 & Berosus & 8 \\
\hline ATG_132 & $\begin{array}{l}\text { Hanging Woman Creek county road } \\
\text { crossing OW Ranch \#2 }\end{array}$ & 7/9/2010 & EMAP_R500 & Callibaetis & 22 \\
\hline ATG_132 & $\begin{array}{l}\text { Hanging Woman Creek county road } \\
\text { crossing OW Ranch \#2 }\end{array}$ & 7/9/2010 & EMAP_R500 & Chaoborus & 247 \\
\hline ATG_132 & $\begin{array}{l}\text { Hanging Woman Creek county road } \\
\text { crossing OW Ranch \#2 }\end{array}$ & 7/9/2010 & EMAP_R500 & Chironomus & 26 \\
\hline ATG_132 & $\begin{array}{l}\text { Hanging Woman Creek county road } \\
\text { crossing OW Ranch \#2 }\end{array}$ & 7/9/2010 & EMAP_R500 & Chrysops & 5 \\
\hline ATG_132 & $\begin{array}{l}\text { Hanging Woman Creek county road } \\
\text { crossing OW Ranch \#2 }\end{array}$ & 7/9/2010 & EMAP_R500 & Corixidae & 66 \\
\hline ATG_132 & $\begin{array}{l}\text { Hanging Woman Creek county road } \\
\text { crossing OW Ranch \#2 }\end{array}$ & 7/9/2010 & EMAP_R500 & Cricotopus & 14 \\
\hline ATG_132 & $\begin{array}{l}\text { Hanging Woman Creek county road } \\
\text { crossing OW Ranch \#2 }\end{array}$ & 7/9/2010 & EMAP_R500 & Culex & 5 \\
\hline ATG_132 & $\begin{array}{l}\text { Hanging Woman Creek county road } \\
\text { crossing OW Ranch \#2 }\end{array}$ & 7/9/2010 & EMAP_R500 & Culicoides & 17 \\
\hline ATG_132 & $\begin{array}{l}\text { Hanging Woman Creek county road } \\
\text { crossing OW Ranch \#2 }\end{array}$ & 7/9/2010 & EMAP_R500 & Enallagma & 6 \\
\hline ATG_132 & $\begin{array}{l}\text { Hanging Woman Creek county road } \\
\text { crossing OW Ranch \#2 }\end{array}$ & 7/9/2010 & EMAP_R500 & Gyraulus parvus & 1 \\
\hline ATG_132 & $\begin{array}{l}\text { Hanging Woman Creek county road } \\
\text { crossing OW Ranch \#2 }\end{array}$ & 7/9/2010 & EMAP_R500 & Haliplus & 1 \\
\hline ATG_132 & $\begin{array}{l}\text { Hanging Woman Creek county road } \\
\text { crossing OW Ranch \#2 }\end{array}$ & 7/9/2010 & EMAP_R500 & Helophorus & 12 \\
\hline ATG_132 & $\begin{array}{l}\text { Hanging Woman Creek county road } \\
\text { crossing OW Ranch \#2 }\end{array}$ & $7 / 9 / 2010$ & EMAP_R500 & Hydrobius & 6 \\
\hline ATG_132 & $\begin{array}{l}\text { Hanging Woman Creek county road } \\
\text { crossing OW Ranch \#2 }\end{array}$ & 7/9/2010 & EMAP_R500 & Hydroporus & 4 \\
\hline ATG_132 & $\begin{array}{l}\text { Hanging Woman Creek county road } \\
\text { crossing OW Ranch \#2 }\end{array}$ & 7/9/2010 & EMAP_R500 & Hygrotus & 1 \\
\hline ATG_132 & $\begin{array}{l}\text { Hanging Woman Creek county road } \\
\text { crossing OW Ranch \#2 }\end{array}$ & 7/9/2010 & EMAP_R500 & Illybius & 4 \\
\hline ATG_132 & $\begin{array}{l}\text { Hanging Woman Creek county road } \\
\text { crossing OW Ranch \#2 }\end{array}$ & 7/9/2010 & EMAP_R500 & Lestes & 20 \\
\hline
\end{tabular}




\begin{tabular}{|c|c|c|c|c|c|}
\hline StationID & Site_Name & $\begin{array}{l}\text { Start } \\
\text { Date }\end{array}$ & Activity & Taxon & Abund \\
\hline ATG_132 & $\begin{array}{l}\text { Hanging Woman Creek county road } \\
\text { crossing OW Ranch \#2 }\end{array}$ & $7 / 9 / 2010$ & EMAP_R500 & Octhebius & 1 \\
\hline ATG_132 & $\begin{array}{l}\text { Hanging Woman Creek county road } \\
\text { crossing OW Ranch \#2 }\end{array}$ & $7 / 9 / 2010$ & EMAP_R500 & Orthocladiinae & 72 \\
\hline ATG_132 & $\begin{array}{l}\text { Hanging Woman Creek county road } \\
\text { crossing OW Ranch \#2 }\end{array}$ & $7 / 9 / 2010$ & EMAP_R500 & Probezzia & 3 \\
\hline ATG_132 & $\begin{array}{l}\text { Hanging Woman Creek county road } \\
\text { crossing OW Ranch \#2 }\end{array}$ & $7 / 9 / 2010$ & EMAP_R500 & Procladius & 2 \\
\hline ATG_132 & $\begin{array}{l}\text { Hanging Woman Creek county road } \\
\text { crossing OW Ranch \#2 }\end{array}$ & $7 / 9 / 2010$ & EMAP_R500 & Sympetrum & 3 \\
\hline ATG_132 & $\begin{array}{l}\text { Hanging Woman Creek county road } \\
\text { crossing OW Ranch } \# 2\end{array}$ & $7 / 9 / 2010$ & EMAP_R500 & Tropisternus lateralis & 3 \\
\hline ATG_132 & $\begin{array}{l}\text { Hanging Woman Creek county road } \\
\text { crossing OW Ranch \#2 }\end{array}$ & $7 / 9 / 2010$ & EMAP_R500 & Tubificidae & 9 \\
\hline ATG_134 & Hanging Woman Creek WP \#134 & $7 / 9 / 2010$ & EMAP_R500 & Stagnicola caperata & 61 \\
\hline ATG_134 & Hanging Woman Creek WP \#134 & $7 / 9 / 2010$ & EMAP_R500 & Physella acuta & 122 \\
\hline ATG_134 & Hanging Woman Creek WP \#134 & $7 / 9 / 2010$ & EMAP_R500 & Sympetrum & 8 \\
\hline ATG_134 & Hanging Woman Creek WP \#134 & $7 / 9 / 2010$ & EMAP_R500 & Coenagrion & 1 \\
\hline ATG_134 & Hanging Woman Creek WP \#134 & $7 / 9 / 2010$ & EMAP_R500 & Ishnura & 6 \\
\hline ATG_134 & Hanging Woman Creek WP \#134 & $7 / 9 / 2010$ & EMAP_R500 & Lestes & 34 \\
\hline ATG_134 & Hanging Woman Creek WP \#134 & $7 / 9 / 2010$ & EMAP_R500 & cricotopus & 43 \\
\hline ATG_134 & Hanging Woman Creek WP \#134 & $7 / 9 / 2010$ & EMAP_R500 & Aeshna & 5 \\
\hline ATG_134 & Hanging Woman Creek WP \#134 & 7/9/2010 & EMAP_R500 & Callibaetis & 54 \\
\hline ATG_134 & Hanging Woman Creek WP \#134 & 7/9/2010 & EMAP_R500 & Psectrocladius & 3 \\
\hline ATG_134 & Hanging Woman Creek WP \#134 & 7/9/2010 & EMAP_R500 & Ostracoda & 32 \\
\hline ATG_134 & Hanging Woman Creek WP \#134 & $7 / 9 / 2010$ & EMAP_R500 & Haliplus & 6 \\
\hline ATG_134 & Hanging Woman Creek WP \#134 & 7/9/2010 & EMAP_R500 & Odontomyia & 6 \\
\hline ATG_134 & Hanging Woman Creek WP \#134 & $7 / 9 / 2010$ & EMAP_R500 & Corynoneura & 13 \\
\hline ATG_134 & Hanging Woman Creek WP \#134 & 7/9/2010 & EMAP_R500 & Corixidae & 28 \\
\hline ATG_134 & Hanging Woman Creek WP \#134 & $7 / 9 / 2010$ & EMAP_R500 & Helophorus & 1 \\
\hline ATG_134 & Hanging Woman Creek WP \#134 & $7 / 9 / 2010$ & EMAP_R500 & Hydroporus & 2 \\
\hline ATG_134 & Hanging Woman Creek WP \#134 & $7 / 9 / 2010$ & EMAP_R500 & Paratanytarsus & 29 \\
\hline ATG_134 & Hanging Woman Creek WP \#134 & 7/9/2010 & EMAP_R500 & Polypedilum & 9 \\
\hline ATG_134 & Hanging Woman Creek WP \#134 & 7/9/2010 & EMAP_R500 & Enallagma & 22 \\
\hline ATG_134 & Hanging Woman Creek WP \#134 & 7/9/2010 & EMAP_R500 & Tanypodinae & 30 \\
\hline ATG_137 & Hanging Woman Creek WP \#137 & $7 / 9 / 2010$ & EMAP_R500 & Lestes & 13 \\
\hline ATG_137 & Hanging Woman Creek WP \#137 & 7/9/2010 & EMAP_R500 & Hyalella azteca & 65 \\
\hline ATG_137 & Hanging Woman Creek WP \#137 & $7 / 9 / 2010$ & EMAP_R500 & Aeshna & 10 \\
\hline
\end{tabular}

Appendix $B$ - 3 


\begin{tabular}{|c|c|c|c|c|c|}
\hline StationID & Site_Name & $\begin{array}{l}\text { Start } \\
\text { Date }\end{array}$ & Activity & Taxon & Abund \\
\hline ATG_137 & Hanging Woman Creek WP \#137 & $7 / 9 / 2010$ & EMAP_R500 & Physella acuta & 268 \\
\hline ATG_137 & Hanging Woman Creek WP \#137 & $7 / 9 / 2010$ & EMAP_R500 & Buenoa & 3 \\
\hline ATG_137 & Hanging Woman Creek WP \#137 & $7 / 9 / 2010$ & EMAP_R500 & Libellula & 1 \\
\hline ATG_137 & Hanging Woman Creek WP \#137 & $7 / 9 / 2010$ & EMAP_R500 & Fossaria humilis & 11 \\
\hline ATG_137 & Hanging Woman Creek WP \#137 & $7 / 9 / 2010$ & EMAP_R500 & Tubificidae & 7 \\
\hline ATG_137 & Hanging Woman Creek WP \#137 & $7 / 9 / 2010$ & EMAP_R500 & Corixidae & 3 \\
\hline ATG_137 & Hanging Woman Creek WP \#137 & $7 / 9 / 2010$ & EMAP_R500 & Chironomus & 20 \\
\hline ATG_137 & Hanging Woman Creek WP \#137 & $7 / 9 / 2010$ & EMAP_R500 & Psectrocladius & 23 \\
\hline ATG_137 & Hanging Woman Creek WP \#137 & $7 / 9 / 2010$ & EMAP_R500 & Procladius & 14 \\
\hline ATG_137 & Hanging Woman Creek WP \#137 & $7 / 9 / 2010$ & EMAP_R500 & Berosus & 4 \\
\hline ATG_137 & Hanging Woman Creek WP \#137 & $7 / 9 / 2010$ & EMAP_R500 & Paratanytarsus & 22 \\
\hline ATG_137 & Hanging Woman Creek WP \#137 & $7 / 9 / 2010$ & EMAP_R500 & Haliplus & 12 \\
\hline ATG_137 & Hanging Woman Creek WP \#137 & $7 / 9 / 2010$ & EMAP_R500 & Brychius & 5 \\
\hline ATG_137 & Hanging Woman Creek WP \#137 & $7 / 9 / 2010$ & EMAP_R500 & Sympetrum & 3 \\
\hline ATG_137 & Hanging Woman Creek WP \#137 & $7 / 9 / 2010$ & EMAP_R500 & Enallagma & 8 \\
\hline ATG_137 & Hanging Woman Creek WP \#137 & $7 / 9 / 2010$ & EMAP_R500 & Callibaetis & 6 \\
\hline ATG_141 & $\begin{array}{l}\text { Hanging Woman Creek Private } \\
\text { Forks Ranch WY border site\#1 }\end{array}$ & $7 / 9 / 2010$ & EMAP_R500 & Aeshna eremita & 18 \\
\hline ATG_141 & $\begin{array}{l}\text { Hanging Woman Creek Private } \\
\text { Forks Ranch WY border site\#1 }\end{array}$ & $7 / 9 / 2010$ & EMAP_R500 & Aeshna palmata & 52 \\
\hline ATG_141 & $\begin{array}{l}\text { Hanging Woman Creek Private } \\
\text { Forks Ranch WY border site\#1 }\end{array}$ & $7 / 9 / 2010$ & EMAP_R500 & Berosus & 14 \\
\hline ATG_141 & $\begin{array}{l}\text { Hanging Woman Creek Private } \\
\text { Forks Ranch WY border site\#1 }\end{array}$ & $7 / 9 / 2010$ & EMAP_R500 & Buenoa & 4 \\
\hline ATG_141 & $\begin{array}{l}\text { Hanging Woman Creek Private } \\
\text { Forks Ranch WY border site\#1 }\end{array}$ & $7 / 9 / 2010$ & EMAP_R500 & Callibaetis & 12 \\
\hline ATG_141 & $\begin{array}{l}\text { Hanging Woman Creek Private } \\
\text { Forks Ranch WY border site\#1 }\end{array}$ & $7 / 9 / 2010$ & EMAP_R500 & Chironominae & 58 \\
\hline ATG_141 & $\begin{array}{l}\text { Hanging Woman Creek Private } \\
\text { Forks Ranch WY border site\#1 }\end{array}$ & $7 / 9 / 2010$ & EMAP_R500 & Corixidae & 3 \\
\hline ATG_141 & $\begin{array}{l}\text { Hanging Woman Creek Private } \\
\text { Forks Ranch WY border site\#1 }\end{array}$ & $7 / 9 / 2010$ & EMAP_R500 & Enallagma & 22 \\
\hline ATG_141 & $\begin{array}{l}\text { Hanging Woman Creek Private } \\
\text { Forks Ranch WY border site\#1 }\end{array}$ & 7/9/2010 & EMAP_R500 & Enochrus & 1 \\
\hline ATG_141 & $\begin{array}{l}\text { Hanging Woman Creek Private } \\
\text { Forks Ranch WY border site\#1 }\end{array}$ & $7 / 9 / 2010$ & EMAP_R500 & Haliplus & 26 \\
\hline ATG_141 & $\begin{array}{l}\text { Hanging Woman Creek Private } \\
\text { Forks Ranch WY border site\#1 }\end{array}$ & 7/9/2010 & EMAP_R500 & Helophorus & 4 \\
\hline ATG_141 & $\begin{array}{l}\text { Hanging Woman Creek Private } \\
\text { Forks Ranch WY border site\# } 1\end{array}$ & $7 / 9 / 2010$ & EMAP_R500 & Hyalella azteca & 46 \\
\hline
\end{tabular}




\begin{tabular}{|c|c|c|c|c|c|}
\hline StationID & Site_Name & $\begin{array}{l}\text { Start } \\
\text { Date }\end{array}$ & Activity & Taxon & Abund \\
\hline ATG_141 & $\begin{array}{l}\text { Hanging Woman Creek Private } \\
\text { Forks Ranch WY border site\#1 }\end{array}$ & 7/9/2010 & EMAP_R500 & Hydrobius & 2 \\
\hline ATG_141 & $\begin{array}{l}\text { Hanging Woman Creek Private } \\
\text { Forks Ranch WY border site\#1 }\end{array}$ & 7/9/2010 & EMAP_R500 & Hygrotus & 2 \\
\hline ATG_141 & $\begin{array}{l}\text { Hanging Woman Creek Private } \\
\text { Forks Ranch WY border site\#1 }\end{array}$ & 7/9/2010 & EMAP_R500 & Hygrotus sp. 2 & 2 \\
\hline ATG_141 & $\begin{array}{l}\text { Hanging Woman Creek Private } \\
\text { Forks Ranch WY border site\#1 }\end{array}$ & $7 / 9 / 2010$ & EMAP_R500 & Lestes & 82 \\
\hline ATG_141 & $\begin{array}{l}\text { Hanging Woman Creek Private } \\
\text { Forks Ranch WY border site\#1 }\end{array}$ & $7 / 9 / 2010$ & EMAP_R500 & Libellula & 10 \\
\hline ATG_141 & $\begin{array}{l}\text { Hanging Woman Creek Private } \\
\text { Forks Ranch WY border site\#1 }\end{array}$ & $7 / 9 / 2010$ & EMAP_R500 & Orthocladiinae & 18 \\
\hline ATG_141 & $\begin{array}{l}\text { Hanging Woman Creek Private } \\
\text { Forks Ranch WY border site\#1 }\end{array}$ & 7/9/2010 & EMAP_R500 & Physella acuta & 64 \\
\hline ATG_141 & $\begin{array}{l}\text { Hanging Woman Creek Private } \\
\text { Forks Ranch WY border site\#1 }\end{array}$ & 7/9/2010 & EMAP_R500 & Procladius & 12 \\
\hline ATG_141 & $\begin{array}{l}\text { Hanging Woman Creek Private } \\
\text { Forks Ranch WY border site\#1 }\end{array}$ & $7 / 9 / 2010$ & EMAP_R500 & Sympetrum & 52 \\
\hline ATG_141 & $\begin{array}{l}\text { Hanging Woman Creek Private } \\
\text { Forks Ranch WY border site\#1 }\end{array}$ & 7/9/2010 & EMAP_R500 & Tanypodinae & 12 \\
\hline ATG_141 & $\begin{array}{l}\text { Hanging Woman Creek Private } \\
\text { Forks Ranch WY border site\#1 }\end{array}$ & 7/9/2010 & EMAP_R500 & Tubificidae & 2 \\
\hline ATG_143 & $\begin{array}{l}\text { Hanging Woman Creek Private } \\
\text { Forks Ranch WY border site\#1 }\end{array}$ & 7/9/2010 & EMAP_R500 & Aeshna & 24 \\
\hline ATG_143 & $\begin{array}{l}\text { Hanging Woman Creek Private } \\
\text { Forks Ranch WY border site\#1 }\end{array}$ & 7/9/2010 & EMAP_R500 & Berosus & 11 \\
\hline ATG_143 & $\begin{array}{l}\text { Hanging Woman Creek Private } \\
\text { Forks Ranch WY border site\#1 }\end{array}$ & 7/9/2010 & EMAP_R500 & Buenoa & 4 \\
\hline ATG_143 & $\begin{array}{l}\text { Hanging Woman Creek Private } \\
\text { Forks Ranch WY border site\#1 }\end{array}$ & 7/9/2010 & EMAP_R500 & Callibaetis & 33 \\
\hline ATG_143 & $\begin{array}{l}\text { Hanging Woman Creek Private } \\
\text { Forks Ranch WY border site\#1 }\end{array}$ & $7 / 9 / 2010$ & EMAP_R500 & Chironominae & 24 \\
\hline ATG_143 & $\begin{array}{l}\text { Hanging Woman Creek Private } \\
\text { Forks Ranch WY border site\#1 }\end{array}$ & $7 / 9 / 2010$ & EMAP_R500 & Corixidae & 3 \\
\hline ATG_143 & $\begin{array}{l}\text { Hanging Woman Creek Private } \\
\text { Forks Ranch WY border site\#1 }\end{array}$ & $7 / 9 / 2010$ & EMAP_R500 & Enallagma & 14 \\
\hline ATG_143 & $\begin{array}{l}\text { Hanging Woman Creek Private } \\
\text { Forks Ranch WY border site\#1 }\end{array}$ & 7/9/2010 & EMAP_R500 & Enochrus & 1 \\
\hline ATG_143 & $\begin{array}{l}\text { Hanging Woman Creek Private } \\
\text { Forks Ranch WY border site\#1 }\end{array}$ & $7 / 9 / 2010$ & EMAP_R500 & Haliplus & 12 \\
\hline ATG_143 & $\begin{array}{l}\text { Hanging Woman Creek Private } \\
\text { Forks Ranch WY border site\#1 }\end{array}$ & $7 / 9 / 2010$ & EMAP_R500 & Helophorus & 5 \\
\hline ATG_143 & $\begin{array}{l}\text { Hanging Woman Creek Private } \\
\text { Forks Ranch WY border site\#1 }\end{array}$ & 7/9/2010 & EMAP_R500 & Hyalella azteca & 101 \\
\hline ATG_143 & $\begin{array}{l}\text { Hanging Woman Creek Private } \\
\text { Forks Ranch WY border site\#1 }\end{array}$ & 7/9/2010 & EMAP_R500 & Hydrobius & 2 \\
\hline
\end{tabular}




\begin{tabular}{|c|c|c|c|c|c|}
\hline StationID & Site_Name & $\begin{array}{l}\text { Start } \\
\text { Date }\end{array}$ & Activity & Taxon & Abund \\
\hline ATG_143 & $\begin{array}{l}\text { Hanging Woman Creek Private } \\
\text { Forks Ranch WY border site\#1 }\end{array}$ & 7/9/2010 & EMAP_R500 & Hygrotus & 2 \\
\hline ATG_143 & $\begin{array}{l}\text { Hanging Woman Creek Private } \\
\text { Forks Ranch WY border site\#1 }\end{array}$ & 7/9/2010 & EMAP_R500 & Hygrotus sp. 2 & 2 \\
\hline ATG_143 & $\begin{array}{l}\text { Hanging Woman Creek Private } \\
\text { Forks Ranch WY border site\#1 }\end{array}$ & 7/9/2010 & EMAP_R500 & Lestes & 65 \\
\hline ATG_143 & $\begin{array}{l}\text { Hanging Woman Creek Private } \\
\text { Forks Ranch WY border site\#1 }\end{array}$ & $7 / 9 / 2010$ & EMAP_R500 & Libellula & 5 \\
\hline ATG_143 & $\begin{array}{l}\text { Hanging Woman Creek Private } \\
\text { Forks Ranch WY border site\#1 }\end{array}$ & $7 / 9 / 2010$ & EMAP_R500 & Orthocladiinae & 27 \\
\hline ATG_143 & $\begin{array}{l}\text { Hanging Woman Creek Private } \\
\text { Forks Ranch WY border site\#1 }\end{array}$ & $7 / 9 / 2010$ & EMAP_R500 & Physella acuta & 89 \\
\hline ATG_143 & $\begin{array}{l}\text { Hanging Woman Creek Private } \\
\text { Forks Ranch WY border site\#1 }\end{array}$ & 7/9/2010 & EMAP_R500 & Procladius & 6 \\
\hline ATG_143 & $\begin{array}{l}\text { Hanging Woman Creek Private } \\
\text { Forks Ranch WY border site\#1 }\end{array}$ & $7 / 9 / 2010$ & EMAP_R500 & Sympetrum & 32 \\
\hline ATG_143 & $\begin{array}{l}\text { Hanging Woman Creek Private } \\
\text { Forks Ranch WY border site\#1 }\end{array}$ & $7 / 9 / 2010$ & EMAP_R500 & Tanypodinae & 34 \\
\hline ATG_143 & $\begin{array}{l}\text { Hanging Woman Creek Private } \\
\text { Forks Ranch WY border site\#1 }\end{array}$ & 7/9/2010 & EMAP_R500 & Tubificidae & 12 \\
\hline ATG_145 & $\begin{array}{l}\text { Hanging Woman Creek Private } \\
\text { Forks Ranch WY border site\#2 }\end{array}$ & 7/9/2010 & EMAP_R500 & Aeshna & 12 \\
\hline ATG_145 & $\begin{array}{l}\text { Hanging Woman Creek Private } \\
\text { Forks Ranch WY border site\#2 }\end{array}$ & 7/9/2010 & EMAP_R500 & Callibaetis & 32 \\
\hline ATG_145 & $\begin{array}{l}\text { Hanging Woman Creek Private } \\
\text { Forks Ranch WY border site\#2 }\end{array}$ & 7/9/2010 & EMAP_R500 & Coenagrion & 4 \\
\hline ATG_145 & $\begin{array}{l}\text { Hanging Woman Creek Private } \\
\text { Forks Ranch WY border site\#2 }\end{array}$ & 7/9/2010 & EMAP_R500 & Corixidae & 32 \\
\hline ATG_145 & $\begin{array}{l}\text { Hanging Woman Creek Private } \\
\text { Forks Ranch WY border site\#2 }\end{array}$ & 7/9/2010 & EMAP_R500 & Corynoneura & 24 \\
\hline ATG_145 & $\begin{array}{l}\text { Hanging Woman Creek Private } \\
\text { Forks Ranch WY border site\#2 }\end{array}$ & $7 / 9 / 2010$ & EMAP_R500 & Cricotopus & 80 \\
\hline ATG_145 & $\begin{array}{l}\text { Hanging Woman Creek Private } \\
\text { Forks Ranch WY border site\#2 }\end{array}$ & $7 / 9 / 2010$ & EMAP_R500 & Haliplus & 12 \\
\hline ATG_145 & $\begin{array}{l}\text { Hanging Woman Creek Private } \\
\text { Forks Ranch WY border site\#2 }\end{array}$ & $7 / 9 / 2010$ & EMAP_R500 & Helophorus & 4 \\
\hline ATG_145 & $\begin{array}{l}\text { Hanging Woman Creek Private } \\
\text { Forks Ranch WY border site\#2 }\end{array}$ & 7/9/2010 & EMAP_R500 & Hydroporus & 8 \\
\hline ATG_145 & $\begin{array}{l}\text { Hanging Woman Creek Private } \\
\text { Forks Ranch WY border site\#2 }\end{array}$ & $7 / 9 / 2010$ & EMAP_R500 & Ischnura & 24 \\
\hline ATG_145 & $\begin{array}{l}\text { Hanging Woman Creek Private } \\
\text { Forks Ranch WY border site\#2 }\end{array}$ & $7 / 9 / 2010$ & EMAP_R500 & Lestes & 68 \\
\hline ATG_145 & $\begin{array}{l}\text { Hanging Woman Creek Private } \\
\text { Forks Ranch WY border site\#2 }\end{array}$ & 7/9/2010 & EMAP_R500 & Odontomyia & 12 \\
\hline ATG_145 & $\begin{array}{l}\text { Hanging Woman Creek Private } \\
\text { Forks Ranch WY border site\#2 }\end{array}$ & $7 / 9 / 2010$ & EMAP_R500 & Ostracoda & 32 \\
\hline
\end{tabular}




\begin{tabular}{|c|c|c|c|c|c|}
\hline StationID & Site_Name & $\begin{array}{l}\text { Start } \\
\text { Date }\end{array}$ & Activity & Taxon & Abund \\
\hline ATG_145 & $\begin{array}{l}\text { Hanging Woman Creek Private } \\
\text { Forks Ranch WY border site\#2 }\end{array}$ & 7/9/2010 & EMAP_R500 & Paratanytarsus & 36 \\
\hline ATG_145 & $\begin{array}{l}\text { Hanging Woman Creek Private } \\
\text { Forks Ranch WY border site\#2 }\end{array}$ & 7/9/2010 & EMAP_R500 & Physella acuta & 92 \\
\hline ATG_145 & $\begin{array}{l}\text { Hanging Woman Creek Private } \\
\text { Forks Ranch WY border site\#2 }\end{array}$ & 7/9/2010 & EMAP_R500 & Polypedilum & 10 \\
\hline ATG_145 & $\begin{array}{l}\text { Hanging Woman Creek Private } \\
\text { Forks Ranch WY border site\#2 }\end{array}$ & 7/9/2010 & EMAP_R500 & Psectrocladius & 12 \\
\hline ATG_145 & $\begin{array}{l}\text { Hanging Woman Creek Private } \\
\text { Forks Ranch WY border site\#2 }\end{array}$ & 7/9/2010 & EMAP_R500 & Sympetrum & 32 \\
\hline ATG_150 & $\begin{array}{l}\text { Hanging Woman Creek Private } \\
\text { Forks Ranch } \# 150\end{array}$ & 7/9/2010 & EMAP_R500 & Ablabesmyia & 7 \\
\hline ATG_150 & $\begin{array}{l}\text { Hanging Woman Creek Private } \\
\text { Forks Ranch } \# 150\end{array}$ & 7/9/2010 & EMAP_R500 & Aeshna & 11 \\
\hline ATG_150 & $\begin{array}{l}\text { Hanging Woman Creek Private } \\
\text { Forks Ranch } \# 150\end{array}$ & 7/9/2010 & EMAP_R500 & Berosus & 8 \\
\hline ATG_150 & $\begin{array}{l}\text { Hanging Woman Creek Private } \\
\text { Forks Ranch } \# 150\end{array}$ & 7/9/2010 & EMAP_R500 & Buenoa & 7 \\
\hline ATG_150 & $\begin{array}{l}\text { Hanging Woman Creek Private } \\
\text { Forks Ranch } \# 150\end{array}$ & 7/9/2010 & EMAP_R500 & Callibaetis & 20 \\
\hline ATG_150 & $\begin{array}{l}\text { Hanging Woman Creek Private } \\
\text { Forks Ranch } \# 150\end{array}$ & 7/9/2010 & EMAP_R500 & Chironomus & 9 \\
\hline ATG_150 & $\begin{array}{l}\text { Hanging Woman Creek Private } \\
\text { Forks Ranch } \# 150\end{array}$ & 7/9/2010 & EMAP_R500 & Corixidae & 24 \\
\hline ATG_150 & $\begin{array}{l}\text { Hanging Woman Creek Private } \\
\text { Forks Ranch } \# 150\end{array}$ & 7/9/2010 & EMAP_R500 & Enallagma & 15 \\
\hline ATG_150 & $\begin{array}{l}\text { Hanging Woman Creek Private } \\
\text { Forks Ranch } \# 150\end{array}$ & 7/9/2010 & EMAP_R500 & Fossaria humilis & 25 \\
\hline ATG_150 & $\begin{array}{l}\text { Hanging Woman Creek Private } \\
\text { Forks Ranch } \# 150\end{array}$ & 7/9/2010 & EMAP_R500 & Haliplus & 9 \\
\hline ATG_150 & $\begin{array}{l}\text { Hanging Woman Creek Private } \\
\text { Forks Ranch \#150 }\end{array}$ & 7/9/2010 & EMAP_R500 & Hyalella azteca & 93 \\
\hline ATG_150 & $\begin{array}{l}\text { Hanging Woman Creek Private } \\
\text { Forks Ranch } \# 150\end{array}$ & 7/9/2010 & EMAP_R500 & Hydroporus & 5 \\
\hline ATG_150 & $\begin{array}{l}\text { Hanging Woman Creek Private } \\
\text { Forks Ranch } \# 150\end{array}$ & 7/9/2010 & EMAP_R500 & Lestes & 23 \\
\hline ATG_150 & $\begin{array}{l}\text { Hanging Woman Creek Private } \\
\text { Forks Ranch } \# 150\end{array}$ & $7 / 9 / 2010$ & EMAP_R500 & Libellula & 2 \\
\hline ATG_150 & $\begin{array}{l}\text { Hanging Woman Creek Private } \\
\text { Forks Ranch } \# 150\end{array}$ & $7 / 9 / 2010$ & EMAP_R500 & Paratanytarsus & 19 \\
\hline ATG_150 & $\begin{array}{l}\text { Hanging Woman Creek Private } \\
\text { Forks Ranch } \# 150\end{array}$ & 7/9/2010 & EMAP_R500 & Physella acuta & 177 \\
\hline ATG_150 & $\begin{array}{l}\text { Hanging Woman Creek Private } \\
\text { Forks Ranch } \# 150\end{array}$ & $7 / 9 / 2010$ & EMAP_R500 & Procladius & 17 \\
\hline ATG_150 & $\begin{array}{l}\text { Hanging Woman Creek Private } \\
\text { Forks Ranch } \# 150\end{array}$ & 7/9/2010 & EMAP_R500 & Psectrocladius & 23 \\
\hline
\end{tabular}




\begin{tabular}{|c|c|c|c|c|c|}
\hline StationID & Site_Name & $\begin{array}{l}\text { Start } \\
\text { Date }\end{array}$ & Activity & Taxon & Abund \\
\hline ATG_150 & $\begin{array}{l}\text { Hanging Woman Creek Private } \\
\text { Forks Ranch } \# 150\end{array}$ & $7 / 9 / 2010$ & EMAP_R500 & Sympetrum & 3 \\
\hline ATG_150 & $\begin{array}{l}\text { Hanging Woman Creek Private } \\
\text { Forks Ranch } \# 150\end{array}$ & $7 / 9 / 2010$ & EMAP_R500 & Thienemannimyia gr. & 2 \\
\hline ATG_150 & $\begin{array}{l}\text { Hanging Woman Creek Private } \\
\text { Forks Ranch } \# 150\end{array}$ & $7 / 9 / 2010$ & EMAP_R500 & Tubificidae & 13 \\
\hline ATG_160 & Seventysix Creek trib to Trail Creek & $7 / 10 / 2010$ & EMAP_R500 & Aeshna & 12 \\
\hline ATG_160 & Seventysix Creek trib to Trail Creek & $7 / 10 / 2010$ & EMAP_R500 & Agabus & 3 \\
\hline ATG_160 & Seventysix Creek trib to Trail Creek & 7/10/2010 & EMAP_R500 & Berosus & 2 \\
\hline ATG_160 & Seventysix Creek trib to Trail Creek & $7 / 10 / 2010$ & EMAP_R500 & Buenoa & 3 \\
\hline ATG_160 & Seventysix Creek trib to Trail Creek & $7 / 10 / 2010$ & EMAP_R500 & Callibaetis & 8 \\
\hline ATG_160 & Seventysix Creek trib to Trail Creek & $7 / 10 / 2010$ & EMAP_R500 & chironomus & 8 \\
\hline ATG_160 & Seventysix Creek trib to Trail Creek & $7 / 10 / 2010$ & EMAP_R500 & Cricotopus & 22 \\
\hline ATG_160 & Seventysix Creek trib to Trail Creek & $7 / 10 / 2010$ & EMAP_R500 & Enallagma & 13 \\
\hline ATG_160 & Seventysix Creek trib to Trail Creek & $7 / 10 / 2010$ & EMAP_R500 & Ephydra & 3 \\
\hline ATG_160 & Seventysix Creek trib to Trail Creek & $7 / 10 / 2010$ & EMAP_R500 & Glyptotendipes & 6 \\
\hline ATG_160 & Seventysix Creek trib to Trail Creek & $7 / 10 / 2010$ & EMAP_R500 & Haliplus & 6 \\
\hline ATG_160 & Seventysix Creek trib to Trail Creek & $7 / 10 / 2010$ & EMAP_R500 & Illybius & 4 \\
\hline ATG_160 & Seventysix Creek trib to Trail Creek & $7 / 10 / 2010$ & EMAP_R500 & Lestes & 55 \\
\hline ATG_160 & Seventysix Creek trib to Trail Creek & $7 / 10 / 2010$ & EMAP_R500 & Libelulla & 2 \\
\hline ATG_160 & Seventysix Creek trib to Trail Creek & $7 / 10 / 2010$ & EMAP_R500 & Orthocladiinae & 37 \\
\hline ATG_160 & Seventysix Creek trib to Trail Creek & $7 / 10 / 2010$ & EMAP_R500 & Ostracoda & 16 \\
\hline ATG_160 & Seventysix Creek trib to Trail Creek & $7 / 10 / 2010$ & EMAP_R500 & Paratanytarsus & 11 \\
\hline ATG_160 & Seventysix Creek trib to Trail Creek & $7 / 10 / 2010$ & EMAP_R500 & Physella acuta & 156 \\
\hline ATG_160 & Seventysix Creek trib to Trail Creek & $7 / 10 / 2010$ & EMAP_R500 & Polypedilum & 2 \\
\hline ATG_160 & Seventysix Creek trib to Trail Creek & $7 / 10 / 2010$ & EMAP_R500 & Procladius & 11 \\
\hline ATG_160 & Seventysix Creek trib to Trail Creek & $7 / 10 / 2010$ & EMAP_R500 & Psectrocladius & 66 \\
\hline ATG_160 & Seventysix Creek trib to Trail Creek & $7 / 10 / 2010$ & EMAP_R500 & Stagnicola elodes & 68 \\
\hline ATG_160 & Seventysix Creek trib to Trail Creek & $7 / 10 / 2010$ & EMAP_R500 & Sympetrum & 2 \\
\hline ATG_163 & $\begin{array}{l}\text { Seventysix Creek Lower trib to } \\
\text { Trail Creek }\end{array}$ & $7 / 10 / 2010$ & EMAP_R500 & Aeshna & 15 \\
\hline ATG_163 & $\begin{array}{l}\text { Seventysix Creek Lower trib to } \\
\text { Trail Creek }\end{array}$ & $7 / 10 / 2010$ & EMAP_R500 & Agabus & 3 \\
\hline ATG_163 & $\begin{array}{l}\text { Seventysix Creek Lower trib to } \\
\text { Trail Creek }\end{array}$ & $7 / 10 / 2010$ & EMAP_R500 & Berosus & 12 \\
\hline ATG_163 & $\begin{array}{l}\text { Seventysix Creek Lower trib to } \\
\text { Trail Creek }\end{array}$ & $7 / 10 / 2010$ & EMAP_R500 & Callibaetis & 10 \\
\hline
\end{tabular}




\begin{tabular}{|c|c|c|c|c|c|}
\hline StationID & Site_Name & $\begin{array}{l}\text { Start } \\
\text { Date }\end{array}$ & Activity & Taxon & Abund \\
\hline ATG_163 & $\begin{array}{l}\text { Seventysix Creek Lower trib to } \\
\text { Trail Creek }\end{array}$ & $7 / 10 / 2010$ & EMAP_R500 & Chironomus & 21 \\
\hline ATG_163 & $\begin{array}{l}\text { Seventysix Creek Lower trib to } \\
\text { Trail Creek }\end{array}$ & $7 / 10 / 2010$ & EMAP_R500 & Enallagma & 18 \\
\hline ATG_163 & $\begin{array}{l}\text { Seventysix Creek Lower trib to } \\
\text { Trail Creek }\end{array}$ & $7 / 10 / 2010$ & EMAP_R500 & Fossaria humilis & 35 \\
\hline ATG_163 & $\begin{array}{l}\text { Seventysix Creek Lower trib to } \\
\text { Trail Creek }\end{array}$ & $7 / 10 / 2010$ & EMAP_R500 & Haliplus & 4 \\
\hline ATG_163 & $\begin{array}{l}\text { Seventysix Creek Lower trib to } \\
\text { Trail Creek }\end{array}$ & $7 / 10 / 2010$ & EMAP_R500 & Illybius & 3 \\
\hline ATG_163 & $\begin{array}{l}\text { Seventysix Creek Lower trib to } \\
\text { Trail Creek }\end{array}$ & $7 / 10 / 2010$ & EMAP_R500 & Lestes & 31 \\
\hline ATG_163 & $\begin{array}{l}\text { Seventysix Creek Lower trib to } \\
\text { Trail Creek }\end{array}$ & $7 / 10 / 2010$ & EMAP_R500 & Lestes dryas & 5 \\
\hline ATG_163 & $\begin{array}{l}\text { Seventysix Creek Lower trib to } \\
\text { Trail Creek }\end{array}$ & $7 / 10 / 2010$ & EMAP_R500 & Orthocladiinae & 37 \\
\hline ATG_163 & $\begin{array}{l}\text { Seventysix Creek Lower trib to } \\
\text { Trail Creek }\end{array}$ & $7 / 10 / 2010$ & EMAP_R500 & Ostracoda & 43 \\
\hline ATG_163 & $\begin{array}{l}\text { Seventysix Creek Lower trib to } \\
\text { Trail Creek }\end{array}$ & $7 / 10 / 2010$ & EMAP_R500 & Paratanytarsus & 14 \\
\hline ATG_163 & $\begin{array}{l}\text { Seventysix Creek Lower trib to } \\
\text { Trail Creek }\end{array}$ & $7 / 10 / 2010$ & EMAP_R500 & Physella acuta & 176 \\
\hline ATG_163 & $\begin{array}{l}\text { Seventysix Creek Lower trib to } \\
\text { Trail Creek }\end{array}$ & $7 / 10 / 2010$ & EMAP_R500 & Polypedilum & 20 \\
\hline ATG_163 & $\begin{array}{l}\text { Seventysix Creek Lower trib to } \\
\text { Trail Creek }\end{array}$ & $7 / 10 / 2010$ & EMAP_R500 & Psectrocladius & 66 \\
\hline ATG_163 & $\begin{array}{l}\text { Seventysix Creek Lower trib to } \\
\text { Trail Creek }\end{array}$ & $7 / 10 / 2010$ & EMAP_R500 & Sympetrum & 6 \\
\hline ATG_179 & Trail Creek \#179 & $7 / 10 / 2010$ & EMAP_R500 & Aedes & 4 \\
\hline ATG_179 & Trail Creek \#179 & $7 / 10 / 2010$ & EMAP_R500 & Aeshna & 10 \\
\hline ATG_179 & Trail Creek \#179 & $7 / 10 / 2010$ & EMAP_R500 & Agabus & 1 \\
\hline ATG_179 & Trail Creek \#179 & $7 / 10 / 2010$ & EMAP_R500 & Cricotopus & 50 \\
\hline ATG_179 & Trail Creek \#179 & $7 / 10 / 2010$ & EMAP_R500 & Culicoides & 2 \\
\hline ATG_179 & Trail Creek \#179 & $7 / 10 / 2010$ & EMAP_R500 & Enallagma & 2 \\
\hline ATG_179 & Trail Creek \#179 & $7 / 10 / 2010$ & EMAP_R500 & Fossaria humilis & 13 \\
\hline ATG_179 & Trail Creek \#179 & $7 / 10 / 2010$ & EMAP_R500 & Haliplus & 1 \\
\hline ATG_179 & Trail Creek \#179 & $7 / 10 / 2010$ & EMAP_R500 & Helophorus & 1 \\
\hline ATG_179 & Trail Creek \#179 & 7/10/2010 & EMAP_R500 & Hyalella azteca & 25 \\
\hline ATG_179 & Trail Creek \#179 & $7 / 10 / 2010$ & EMAP_R500 & Hydrobius & 1 \\
\hline ATG_179 & Trail Creek \#179 & 7/10/2010 & EMAP_R500 & Illybius & 1 \\
\hline ATG_179 & Trail Creek \#179 & $7 / 10 / 2010$ & EMAP_R500 & Lestes & 15 \\
\hline
\end{tabular}




\begin{tabular}{|c|c|c|c|c|c|}
\hline StationID & Site_Name & $\begin{array}{l}\text { Start } \\
\text { Date }\end{array}$ & Activity & Taxon & Abund \\
\hline ATG_179 & Trail Creek \#179 & $7 / 10 / 2010$ & EMAP_R500 & Octhebius & 2 \\
\hline ATG_179 & Trail Creek \#179 & $7 / 10 / 2010$ & EMAP_R500 & Ostracoda & 22 \\
\hline ATG_179 & Trail Creek \#179 & $7 / 10 / 2010$ & EMAP_R500 & Paratanytarsus & 2 \\
\hline ATG_179 & Trail Creek \#179 & $7 / 10 / 2010$ & EMAP_R500 & Peltodytes & 1 \\
\hline ATG_179 & Trail Creek \#179 & $7 / 10 / 2010$ & EMAP_R500 & Psectrocladius & 17 \\
\hline ATG_179 & Trail Creek \#179 & $7 / 10 / 2010$ & EMAP_R500 & Stagnicola elodes & 330 \\
\hline ATG_179 & Trail Creek \#179 & $7 / 10 / 2010$ & EMAP_R500 & Sympetrum & 13 \\
\hline ATG_179 & Trail Creek \#179 & $7 / 10 / 2010$ & EMAP_R500 & Tanypodinae & 3 \\
\hline ATG_179 & Trail Creek \#179 & $7 / 10 / 2010$ & EMAP_R500 & Tubificidae & 2 \\
\hline ATG_196 & $\begin{array}{l}\text { Hanging Woman Creek OW Ranch } \\
\text { bridge site }\end{array}$ & $7 / 10 / 2010$ & EMAP_R500 & Aeshna eremita & 1 \\
\hline ATG_196 & $\begin{array}{l}\text { Hanging Woman Creek OW Ranch } \\
\text { bridge site }\end{array}$ & $7 / 10 / 2010$ & EMAP_R500 & Aeshna palmata & 1 \\
\hline ATG_196 & $\begin{array}{l}\text { Hanging Woman Creek OW Ranch } \\
\text { bridge site }\end{array}$ & $7 / 10 / 2010$ & EMAP_R500 & Berosus & 14 \\
\hline ATG_196 & $\begin{array}{l}\text { Hanging Woman Creek OW Ranch } \\
\text { bridge site }\end{array}$ & $7 / 10 / 2010$ & EMAP_R500 & Bиепоа & 1 \\
\hline ATG_196 & $\begin{array}{l}\text { Hanging Woman Creek OW Ranch } \\
\text { bridge site }\end{array}$ & $7 / 10 / 2010$ & EMAP_R500 & Caenis latipennis & 1 \\
\hline ATG_196 & $\begin{array}{l}\text { Hanging Woman Creek OW Ranch } \\
\text { bridge site }\end{array}$ & $7 / 10 / 2010$ & EMAP_R500 & Callibaetis & 81 \\
\hline ATG_196 & $\begin{array}{l}\text { Hanging Woman Creek OW Ranch } \\
\text { bridge site }\end{array}$ & $7 / 10 / 2010$ & EMAP_R500 & Chironominae & 50 \\
\hline ATG_196 & $\begin{array}{l}\text { Hanging Woman Creek OW Ranch } \\
\text { bridge site }\end{array}$ & $7 / 10 / 2010$ & EMAP_R500 & Corixidae & 32 \\
\hline ATG_196 & $\begin{array}{l}\text { Hanging Woman Creek OW Ranch } \\
\text { bridge site }\end{array}$ & $7 / 10 / 2010$ & EMAP_R500 & Culicoides & 1 \\
\hline ATG_196 & $\begin{array}{l}\text { Hanging Woman Creek OW Ranch } \\
\text { bridge site }\end{array}$ & $7 / 10 / 2010$ & EMAP_R500 & Dolichopodidae & 1 \\
\hline ATG_196 & $\begin{array}{l}\text { Hanging Woman Creek OW Ranch } \\
\text { bridge site }\end{array}$ & $7 / 10 / 2010$ & EMAP_R500 & enallagma & 6 \\
\hline ATG_196 & $\begin{array}{l}\text { Hanging Woman Creek OW Ranch } \\
\text { bridge site }\end{array}$ & $7 / 10 / 2010$ & EMAP_R500 & Haliplus & 1 \\
\hline ATG_196 & $\begin{array}{l}\text { Hanging Woman Creek OW Ranch } \\
\text { bridge site }\end{array}$ & $7 / 10 / 2010$ & EMAP_R500 & Helophorus & 1 \\
\hline ATG_196 & $\begin{array}{l}\text { Hanging Woman Creek OW Ranch } \\
\text { bridge site }\end{array}$ & $7 / 10 / 2010$ & EMAP_R500 & Hyalella azteca & 51 \\
\hline ATG_196 & $\begin{array}{l}\text { Hanging Woman Creek OW Ranch } \\
\text { bridge site }\end{array}$ & $7 / 10 / 2010$ & EMAP_R500 & Hydrobius & 1 \\
\hline ATG_196 & $\begin{array}{l}\text { Hanging Woman Creek OW Ranch } \\
\text { bridge site }\end{array}$ & $7 / 10 / 2010$ & EMAP_R500 & Illybius & 6 \\
\hline ATG_196 & $\begin{array}{l}\text { Hanging Woman Creek OW Ranch } \\
\text { bridge site }\end{array}$ & $7 / 10 / 2010$ & EMAP_R500 & Laccophilus & 1 \\
\hline
\end{tabular}

Appendix $B-10$ 


\begin{tabular}{|c|c|c|c|c|c|}
\hline StationID & Site_Name & $\begin{array}{l}\text { Start } \\
\text { Date }\end{array}$ & Activity & Taxon & Abund \\
\hline ATG_196 & $\begin{array}{l}\text { Hanging Woman Creek OW Ranch } \\
\text { bridge site }\end{array}$ & $7 / 10 / 2010$ & EMAP_R500 & Lestes & 19 \\
\hline ATG_196 & $\begin{array}{l}\text { Hanging Woman Creek OW Ranch } \\
\text { bridge site }\end{array}$ & 7/10/2010 & EMAP_R500 & Libellula & 1 \\
\hline ATG_196 & $\begin{array}{l}\text { Hanging Woman Creek OW Ranch } \\
\text { bridge site }\end{array}$ & 7/10/2010 & EMAP_R500 & Orthocladiinae & 7 \\
\hline ATG_196 & $\begin{array}{l}\text { Hanging Woman Creek OW Ranch } \\
\text { bridge site }\end{array}$ & $7 / 10 / 2010$ & EMAP_R500 & Physella acuta & 3 \\
\hline ATG_196 & $\begin{array}{l}\text { Hanging Woman Creek OW Ranch } \\
\text { bridge site }\end{array}$ & 7/10/2010 & EMAP_R500 & Physella gyrina & 1 \\
\hline ATG_196 & $\begin{array}{l}\text { Hanging Woman Creek OW Ranch } \\
\text { bridge site }\end{array}$ & 7/10/2010 & EMAP_R500 & Procladius & 1 \\
\hline ATG_196 & $\begin{array}{l}\text { Hanging Woman Creek OW Ranch } \\
\text { bridge site }\end{array}$ & 7/10/2010 & EMAP_R500 & Simulium & 3 \\
\hline ATG_196 & $\begin{array}{l}\text { Hanging Woman Creek OW Ranch } \\
\text { bridge site }\end{array}$ & $7 / 10 / 2010$ & EMAP_R500 & Stagnicola caperata & 1 \\
\hline ATG_196 & $\begin{array}{l}\text { Hanging Woman Creek OW Ranch } \\
\text { bridge site }\end{array}$ & 7/10/2010 & EMAP_R500 & Sympetrum & 2 \\
\hline ATG_196 & $\begin{array}{l}\text { Hanging Woman Creek OW Ranch } \\
\text { bridge site }\end{array}$ & $7 / 10 / 2010$ & EMAP_R500 & Tabanus & 1 \\
\hline ATG_196 & $\begin{array}{l}\text { Hanging Woman Creek OW Ranch } \\
\text { bridge site }\end{array}$ & $7 / 10 / 2010$ & EMAP_R500 & Tanypodinae & 93 \\
\hline ATG_196 & $\begin{array}{l}\text { Hanging Woman Creek OW Ranch } \\
\text { bridge site }\end{array}$ & $7 / 10 / 2010$ & EMAP_R500 & Tubificidae & 3 \\
\hline ATG_200 & $\begin{array}{l}\text { Hanging Woman Creek at Quietus } \\
\text { Road Bridge upstream }\end{array}$ & $7 / 11 / 2010$ & EMAP_R500 & Ablabesmyia & 8 \\
\hline ATG_200 & $\begin{array}{l}\text { Hanging Woman Creek at Quietus } \\
\text { Road Bridge upstream }\end{array}$ & $7 / 11 / 2010$ & EMAP_R500 & Caenis latipennis & 44 \\
\hline ATG_200 & $\begin{array}{l}\text { Hanging Woman Creek at Quietus } \\
\text { Road Bridge upstream }\end{array}$ & $7 / 11 / 2010$ & EMAP_R500 & Callibaetis & 19 \\
\hline ATG_200 & $\begin{array}{l}\text { Hanging Woman Creek at Quietus } \\
\text { Road Bridge upstream }\end{array}$ & $7 / 11 / 2010$ & EMAP_R500 & Chironomus & 12 \\
\hline ATG_200 & $\begin{array}{l}\text { Hanging Woman Creek at Quietus } \\
\text { Road Bridge upstream }\end{array}$ & $7 / 11 / 2010$ & EMAP_R500 & Coenagrion/Enallagma & 6 \\
\hline ATG_200 & $\begin{array}{l}\text { Hanging Woman Creek at Quietus } \\
\text { Road Bridge upstream }\end{array}$ & $7 / 11 / 2010$ & EMAP_R500 & Coenagrionidae & 10 \\
\hline ATG_200 & $\begin{array}{l}\text { Hanging Woman Creek at Quietus } \\
\text { Road Bridge upstream }\end{array}$ & 7/11/2010 & EMAP_R500 & Corixidae & 58 \\
\hline ATG_200 & $\begin{array}{l}\text { Hanging Woman Creek at Quietus } \\
\text { Road Bridge upstream }\end{array}$ & $7 / 11 / 2010$ & EMAP_R500 & Cricotopus & 39 \\
\hline ATG_200 & $\begin{array}{l}\text { Hanging Woman Creek at Quietus } \\
\text { Road Bridge upstream }\end{array}$ & $7 / 11 / 2010$ & EMAP_R500 & Cricotopus bicinctus gr. & 6 \\
\hline ATG_200 & $\begin{array}{l}\text { Hanging Woman Creek at Quietus } \\
\text { Road Bridge upstream }\end{array}$ & $7 / 11 / 2010$ & EMAP_R500 & Cryptochironomus & 7 \\
\hline ATG_200 & $\begin{array}{l}\text { Hanging Woman Creek at Quietus } \\
\text { Road Bridge upstream }\end{array}$ & 7/11/2010 & EMAP_R500 & Dicrotendipes & 9 \\
\hline
\end{tabular}




\begin{tabular}{|c|c|c|c|c|c|}
\hline StationID & Site_Name & $\begin{array}{l}\text { Start } \\
\text { Date }\end{array}$ & Activity & Taxon & Abund \\
\hline ATG_200 & $\begin{array}{l}\text { Hanging Woman Creek at Quietus } \\
\text { Road Bridge upstream }\end{array}$ & $7 / 11 / 2010$ & EMAP_R500 & Dubiraphia & 6 \\
\hline ATG_200 & $\begin{array}{l}\text { Hanging Woman Creek at Quietus } \\
\text { Road Bridge upstream }\end{array}$ & $7 / 11 / 2010$ & EMAP_R500 & Erpobdellidae & 2 \\
\hline ATG_200 & $\begin{array}{l}\text { Hanging Woman Creek at Quietus } \\
\text { Road Bridge upstream }\end{array}$ & $7 / 11 / 2010$ & EMAP_R500 & Glossiphoniidae & 3 \\
\hline ATG_200 & $\begin{array}{l}\text { Hanging Woman Creek at Quietus } \\
\text { Road Bridge upstream }\end{array}$ & $7 / 11 / 2010$ & EMAP_R500 & Hyalella azteca & 122 \\
\hline ATG_200 & $\begin{array}{l}\text { Hanging Woman Creek at Quietus } \\
\text { Road Bridge upstream }\end{array}$ & 7/11/2010 & EMAP_R500 & Hydroptila & 8 \\
\hline ATG_200 & $\begin{array}{l}\text { Hanging Woman Creek at Quietus } \\
\text { Road Bridge upstream }\end{array}$ & 7/11/2010 & EMAP_R500 & Micropsectra & 15 \\
\hline ATG_200 & $\begin{array}{l}\text { Hanging Woman Creek at Quietus } \\
\text { Road Bridge upstream }\end{array}$ & $7 / 11 / 2010$ & EMAP_R500 & Microtendipes & 4 \\
\hline ATG_200 & $\begin{array}{l}\text { Hanging Woman Creek at Quietus } \\
\text { Road Bridge upstream }\end{array}$ & 7/11/2010 & EMAP_R500 & Nectopsyche & 2 \\
\hline ATG_200 & $\begin{array}{l}\text { Hanging Woman Creek at Quietus } \\
\text { Road Bridge upstream }\end{array}$ & 7/11/2010 & EMAP_R500 & Tubificidae & 12 \\
\hline ATG_200 & $\begin{array}{l}\text { Hanging Woman Creek at Quietus } \\
\text { Road Bridge upstream }\end{array}$ & 7/11/2010 & EMAP_R500 & Oreodytes & 5 \\
\hline ATG_200 & $\begin{array}{l}\text { Hanging Woman Creek at Quietus } \\
\text { Road Bridge upstream }\end{array}$ & 7/11/2010 & EMAP_R500 & Paracladopelma & 3 \\
\hline ATG_200 & $\begin{array}{l}\text { Hanging Woman Creek at Quietus } \\
\text { Road Bridge upstream }\end{array}$ & 7/11/2010 & EMAP_R500 & Physella acuta & 33 \\
\hline ATG_200 & $\begin{array}{l}\text { Hanging Woman Creek at Quietus } \\
\text { Road Bridge upstream }\end{array}$ & $7 / 11 / 2010$ & EMAP_R500 & Sphaerium & 5 \\
\hline ATG_200 & $\begin{array}{l}\text { Hanging Woman Creek at Quietus } \\
\text { Road Bridge upstream }\end{array}$ & $7 / 11 / 2010$ & EMAP_R500 & Procladius & 9 \\
\hline ATG_200 & $\begin{array}{l}\text { Hanging Woman Creek at Quietus } \\
\text { Road Bridge upstream }\end{array}$ & $7 / 11 / 2010$ & EMAP_R500 & Pseudochironomus & 2 \\
\hline ATG_200 & $\begin{array}{l}\text { Hanging Woman Creek at Quietus } \\
\text { Road Bridge upstream }\end{array}$ & $7 / 11 / 2010$ & EMAP_R500 & Paratanytarsus & 16 \\
\hline ATG_200 & $\begin{array}{l}\text { Hanging Woman Creek at Quietus } \\
\text { Road Bridge upstream }\end{array}$ & $7 / 11 / 2010$ & EMAP_R500 & Stagnicola elodes & 5 \\
\hline ATG_200 & $\begin{array}{l}\text { Hanging Woman Creek at Quietus } \\
\text { Road Bridge upstream }\end{array}$ & $7 / 11 / 2010$ & EMAP_R500 & Tanytarsus & 26 \\
\hline ATG_200 & $\begin{array}{l}\text { Hanging Woman Creek at Quietus } \\
\text { Road Bridge upstream }\end{array}$ & 7/11/2010 & EMAP_R500 & Zavrelimyia & 9 \\
\hline ATG_201 & Otter Creek at Tenmile Creek Road & $7 / 11 / 2010$ & EMAP_R500 & Agabus & 12 \\
\hline ATG_201 & Otter Creek at Tenmile Creek Road & $7 / 11 / 2010$ & EMAP_R500 & Ablabesmyia & 5 \\
\hline ATG_201 & Otter Creek at Tenmile Creek Road & $7 / 11 / 2010$ & EMAP_R500 & Acari & 2 \\
\hline ATG_201 & Otter Creek at Tenmile Creek Road & $7 / 11 / 2010$ & EMAP_R500 & Caenis latipennis & 15 \\
\hline ATG_201 & Otter Creek at Tenmile Creek Road & $7 / 11 / 2010$ & EMAP_R500 & Callibaetis & 7 \\
\hline
\end{tabular}




\begin{tabular}{|c|c|c|c|c|c|}
\hline StationID & Site_Name & $\begin{array}{l}\text { Start } \\
\text { Date }\end{array}$ & Activity & Taxon & Abund \\
\hline ATG_201 & Otter Creek at Tenmile Creek Road & 7/11/2010 & EMAP_R500 & Caloparyphus & 1 \\
\hline ATG_201 & Otter Creek at Tenmile Creek Road & $7 / 11 / 2010$ & EMAP_R500 & Chironomus & 23 \\
\hline ATG_201 & Otter Creek at Tenmile Creek Road & 7/11/2010 & EMAP_R500 & Corixidae & 13 \\
\hline ATG_201 & Otter Creek at Tenmile Creek Road & 7/11/2010 & EMAP_R500 & Corynoneura & 6 \\
\hline ATG_201 & Otter Creek at Tenmile Creek Road & 7/11/2010 & EMAP_R500 & Cricotopus bicinctus gr. & 54 \\
\hline ATG_201 & Otter Creek at Tenmile Creek Road & 7/11/2010 & EMAP_R500 & Cricotopus trifascia gr. & 32 \\
\hline ATG_201 & Otter Creek at Tenmile Creek Road & 7/11/2010 & EMAP_R500 & Culicoides & 2 \\
\hline ATG_201 & Otter Creek at Tenmile Creek Road & 7/11/2010 & EMAP_R500 & Dicrotendipes & 7 \\
\hline ATG_201 & Otter Creek at Tenmile Creek Road & $7 / 11 / 2010$ & EMAP_R500 & Dubiraphia & 4 \\
\hline ATG_201 & Otter Creek at Tenmile Creek Road & 7/11/2010 & EMAP_R500 & Glossiphonia complanata & 2 \\
\hline ATG_201 & Otter Creek at Tenmile Creek Road & 7/11/2010 & EMAP_R500 & Gyraulus parvus & 6 \\
\hline ATG_201 & Otter Creek at Tenmile Creek Road & 7/11/2010 & EMAP_R500 & Helisoma anceps & 2 \\
\hline ATG_201 & Otter Creek at Tenmile Creek Road & 7/11/2010 & EMAP_R500 & Hesperophylax designatus & 4 \\
\hline ATG_201 & Otter Creek at Tenmile Creek Road & $7 / 11 / 2010$ & EMAP_R500 & Hyalella azteca & 23 \\
\hline ATG_201 & Otter Creek at Tenmile Creek Road & $7 / 11 / 2010$ & EMAP_R500 & Lestes & 6 \\
\hline ATG_201 & Otter Creek at Tenmile Creek Road & 7/11/2010 & EMAP_R500 & Limnephilus & 8 \\
\hline ATG_201 & Otter Creek at Tenmile Creek Road & $7 / 11 / 2010$ & EMAP_R500 & Micropsectra & 65 \\
\hline ATG_201 & Otter Creek at Tenmile Creek Road & $7 / 11 / 2010$ & EMAP_R500 & Nilotanypus & 2 \\
\hline ATG_201 & Otter Creek at Tenmile Creek Road & 7/11/2010 & EMAP_R500 & Parametriocnemus & 25 \\
\hline ATG_201 & Otter Creek at Tenmile Creek Road & 7/11/2010 & EMAP_R500 & Paratanytarsus & 22 \\
\hline ATG_201 & Otter Creek at Tenmile Creek Road & $7 / 11 / 2010$ & EMAP_R500 & Physella gyrina & 119 \\
\hline ATG_201 & Otter Creek at Tenmile Creek Road & $7 / 11 / 2010$ & EMAP_R500 & Polypedilum & 20 \\
\hline ATG_201 & Otter Creek at Tenmile Creek Road & 7/11/2010 & EMAP_R500 & Procladius & 8 \\
\hline ATG_201 & Otter Creek at Tenmile Creek Road & $7 / 11 / 2010$ & EMAP_R500 & Psectrocladius & 25 \\
\hline ATG_201 & Otter Creek at Tenmile Creek Road & 7/11/2010 & EMAP_R500 & Sciomyzidae & 2 \\
\hline ATG_201 & Otter Creek at Tenmile Creek Road & $7 / 11 / 2010$ & EMAP_R500 & Simulium & 55 \\
\hline ATG_201 & Otter Creek at Tenmile Creek Road & 7/11/2010 & EMAP_R500 & Sympetrum & 1 \\
\hline ATG_201 & Otter Creek at Tenmile Creek Road & $7 / 11 / 2010$ & EMAP_R500 & Tubificidae & 14 \\
\hline ATG_202 & Otter Creek at Taylor Creek Road & $7 / 11 / 2010$ & EMAP_R500 & Agabus & 8 \\
\hline ATG_202 & Otter Creek at Taylor Creek Road & $7 / 11 / 2010$ & EMAP_R500 & Apedilum & 2 \\
\hline ATG_202 & Otter Creek at Taylor Creek Road & $7 / 11 / 2010$ & EMAP_R500 & Baetis tricaudatus & 15 \\
\hline ATG_202 & Otter Creek at Taylor Creek Road & 7/11/2010 & EMAP_R500 & Berosus & 1 \\
\hline ATG_202 & Otter Creek at Taylor Creek Road & $7 / 11 / 2010$ & EMAP_R500 & Bezzia/Palpomyia & 4 \\
\hline ATG_202 & Otter Creek at Taylor Creek Road & 7/11/2010 & |EMAP_R500 & Centroptilum & 2 \\
\hline
\end{tabular}




\begin{tabular}{|c|c|c|c|c|c|}
\hline StationID & Site_Name & $\begin{array}{l}\text { Start } \\
\text { Date }\end{array}$ & Activity & Taxon & Abund \\
\hline ATG_202 & Otter Creek at Taylor Creek Road & $7 / 11 / 2010$ & EMAP_R500 & Cheumatopsyche & 5 \\
\hline ATG_202 & Otter Creek at Taylor Creek Road & $7 / 11 / 2010$ & EMAP_R500 & Chrysops & 4 \\
\hline ATG_202 & Otter Creek at Taylor Creek Road & $7 / 11 / 2010$ & EMAP_R500 & Coptotomus longulus & 1 \\
\hline ATG_202 & Otter Creek at Taylor Creek Road & $7 / 11 / 2010$ & EMAP_R500 & Cricotopus & 14 \\
\hline ATG_202 & Otter Creek at Taylor Creek Road & $7 / 11 / 2010$ & EMAP_R500 & Cricotopus bicinctus gr. & 7 \\
\hline ATG_202 & Otter Creek at Taylor Creek Road & $7 / 11 / 2010$ & EMAP_R500 & Dubiraphia & 2 \\
\hline ATG_202 & Otter Creek at Taylor Creek Road & $7 / 11 / 2010$ & EMAP_R500 & Enallagma & 6 \\
\hline ATG_202 & Otter Creek at Taylor Creek Road & $7 / 11 / 2010$ & EMAP_R500 & Erpobdellidae & 1 \\
\hline ATG_202 & Otter Creek at Taylor Creek Road & $7 / 11 / 2010$ & EMAP_R500 & Eukiefferiella claripennis gr. & 1 \\
\hline ATG_202 & Otter Creek at Taylor Creek Road & $7 / 11 / 2010$ & EMAP_R500 & Gammarus & 12 \\
\hline ATG_202 & Otter Creek at Taylor Creek Road & $7 / 11 / 2010$ & EMAP_R500 & Gyraulus parvus & 6 \\
\hline ATG_202 & Otter Creek at Taylor Creek Road & $7 / 11 / 2010$ & EMAP_R500 & Haliplus & 4 \\
\hline ATG_202 & Otter Creek at Taylor Creek Road & $7 / 11 / 2010$ & EMAP_R500 & Hesperophylax designatus & 12 \\
\hline ATG_202 & Otter Creek at Taylor Creek Road & $7 / 11 / 2010$ & EMAP_R500 & Hyalella azteca & 45 \\
\hline ATG_202 & Otter Creek at Taylor Creek Road & $7 / 11 / 2010$ & EMAP_R500 & Hydrachna & 1 \\
\hline ATG_202 & Otter Creek at Taylor Creek Road & $7 / 11 / 2010$ & EMAP_R500 & Lestes disjunctus & 6 \\
\hline ATG_202 & Otter Creek at Taylor Creek Road & $7 / 11 / 2010$ & EMAP_R500 & Libellula pulchella & 1 \\
\hline ATG_202 & Otter Creek at Taylor Creek Road & $7 / 11 / 2010$ & EMAP_R500 & Limnephilus & 9 \\
\hline ATG_202 & Otter Creek at Taylor Creek Road & $7 / 11 / 2010$ & EMAP_R500 & Ochthebius & 2 \\
\hline ATG_202 & Otter Creek at Taylor Creek Road & $7 / 11 / 2010$ & EMAP_R500 & Optioservus & 2 \\
\hline ATG_202 & Otter Creek at Taylor Creek Road & $7 / 11 / 2010$ & EMAP_R500 & Orconectes virilis & 1 \\
\hline ATG_202 & Otter Creek at Taylor Creek Road & $7 / 11 / 2010$ & EMAP_R500 & Oreodytes & 2 \\
\hline ATG_202 & Otter Creek at Taylor Creek Road & $7 / 11 / 2010$ & EMAP_R500 & Orthocladius & 9 \\
\hline ATG_202 & Otter Creek at Taylor Creek Road & $7 / 11 / 2010$ & EMAP_R500 & Physella gyrina & 176 \\
\hline ATG_202 & Otter Creek at Taylor Creek Road & $7 / 11 / 2010$ & EMAP_R500 & Probezzia & 1 \\
\hline ATG_202 & Otter Creek at Taylor Creek Road & $7 / 11 / 2010$ & EMAP_R500 & Procladius & 4 \\
\hline ATG_202 & Otter Creek at Taylor Creek Road & $7 / 11 / 2010$ & EMAP_R500 & Prodiamesa & 2 \\
\hline ATG_202 & Otter Creek at Taylor Creek Road & $7 / 11 / 2010$ & EMAP_R500 & Simulium & 143 \\
\hline ATG_202 & Otter Creek at Taylor Creek Road & $7 / 11 / 2010$ & EMAP_R500 & Stagnicola & 2 \\
\hline ATG_202 & Otter Creek at Taylor Creek Road & $7 / 11 / 2010$ & EMAP_R500 & Tubificidae & 7 \\
\hline ATG_203 & Otter Creek at Camp Creek Road & 7/11/2010 & EMAP_R500 & Aeshna & 2 \\
\hline ATG_203 & Otter Creek at Camp Creek Road & $7 / 11 / 2010$ & EMAP_R500 & Argia & 12 \\
\hline ATG_203 & Otter Creek at Camp Creek Road & $7 / 11 / 2010$ & EMAP_R500 & Atrichopogon & 3 \\
\hline ATG_203 & Otter Creek at Camp Creek Road & $7 / 11 / 2010$ & EMAP_R500 & Berosus & 12 \\
\hline
\end{tabular}




\begin{tabular}{|c|c|c|c|c|c|}
\hline StationID & Site_Name & $\begin{array}{l}\text { Start } \\
\text { Date }\end{array}$ & Activity & Taxon & Abund \\
\hline ATG_203 & Otter Creek at Camp Creek Road & $7 / 11 / 2010$ & EMAP_R500 & Bezzia & 10 \\
\hline ATG_203 & Otter Creek at Camp Creek Road & $7 / 11 / 2010$ & EMAP_R500 & Caenis latipennis & 122 \\
\hline ATG_203 & Otter Creek at Camp Creek Road & $7 / 11 / 2010$ & EMAP_R500 & Callibaetis ferrugineus & 6 \\
\hline ATG_203 & Otter Creek at Camp Creek Road & $7 / 11 / 2010$ & EMAP_R500 & Ceratopogoninae & 3 \\
\hline ATG_203 & Otter Creek at Camp Creek Road & $7 / 11 / 2010$ & EMAP_R500 & Coenagrion & 32 \\
\hline ATG_203 & Otter Creek at Camp Creek Road & $7 / 11 / 2010$ & EMAP_R500 & Cricotopus bicinctus & 4 \\
\hline ATG_203 & Otter Creek at Camp Creek Road & $7 / 11 / 2010$ & EMAP_R500 & Cryptotendipes & 4 \\
\hline ATG_203 & Otter Creek at Camp Creek Road & $7 / 11 / 2010$ & EMAP_R500 & Dasyhelea & 33 \\
\hline ATG_203 & Otter Creek at Camp Creek Road & $7 / 11 / 2010$ & EMAP_R500 & Dicrotendipes & 21 \\
\hline ATG_203 & Otter Creek at Camp Creek Road & $7 / 11 / 2010$ & EMAP_R500 & Dubiraphia & 3 \\
\hline ATG_203 & Otter Creek at Camp Creek Road & $7 / 11 / 2010$ & EMAP_R500 & Enallagma & 42 \\
\hline ATG_203 & Otter Creek at Camp Creek Road & $7 / 11 / 2010$ & EMAP_R500 & Erpobdellidae & 1 \\
\hline ATG_203 & Otter Creek at Camp Creek Road & $7 / 11 / 2010$ & EMAP_R500 & Gyraulus parvus & 7 \\
\hline ATG_203 & Otter Creek at Camp Creek Road & $7 / 11 / 2010$ & EMAP_R500 & Hyalella azteca & 63 \\
\hline ATG_203 & Otter Creek at Camp Creek Road & $7 / 11 / 2010$ & EMAP_R500 & Orthocladius & 2 \\
\hline ATG_203 & Otter Creek at Camp Creek Road & $7 / 11 / 2010$ & EMAP_R500 & Ostracoda & 45 \\
\hline ATG_203 & Otter Creek at Camp Creek Road & $7 / 11 / 2010$ & EMAP_R500 & Oxyethira & 1 \\
\hline ATG_203 & Otter Creek at Camp Creek Road & $7 / 11 / 2010$ & EMAP_R500 & Parakiefferiella & 6 \\
\hline ATG_203 & Otter Creek at Camp Creek Road & $7 / 11 / 2010$ & EMAP_R500 & Paramerina & 16 \\
\hline ATG_203 & Otter Creek at Camp Creek Road & $7 / 11 / 2010$ & EMAP_R500 & Paratanytarsus & 17 \\
\hline ATG_203 & Otter Creek at Camp Creek Road & $7 / 11 / 2010$ & EMAP_R500 & Paratendipes & 1 \\
\hline ATG_203 & Otter Creek at Camp Creek Road & $7 / 11 / 2010$ & EMAP_R500 & Peltodytes & 1 \\
\hline ATG_203 & Otter Creek at Camp Creek Road & $7 / 11 / 2010$ & EMAP_R500 & Pericoma & 1 \\
\hline ATG_203 & Otter Creek at Camp Creek Road & $7 / 11 / 2010$ & EMAP_R500 & Phaenopsectra & 1 \\
\hline ATG_203 & Otter Creek at Camp Creek Road & $7 / 11 / 2010$ & EMAP_R500 & Physella acuta & 45 \\
\hline ATG_203 & Otter Creek at Camp Creek Road & $7 / 11 / 2010$ & EMAP_R500 & Pisidium & 2 \\
\hline ATG_203 & Otter Creek at Camp Creek Road & $7 / 11 / 2010$ & EMAP_R500 & Polypedilum & 7 \\
\hline ATG_203 & Otter Creek at Camp Creek Road & $7 / 11 / 2010$ & EMAP_R500 & Procladius & 6 \\
\hline ATG_203 & Otter Creek at Camp Creek Road & $7 / 11 / 2010$ & EMAP_R500 & Pseudochironomus & 22 \\
\hline ATG_203 & Otter Creek at Camp Creek Road & $7 / 11 / 2010$ & EMAP_R500 & Simulium & 3 \\
\hline ATG_203 & Otter Creek at Camp Creek Road & 7/11/2010 & EMAP_R500 & Tanytarsus & 27 \\
\hline ATG_203 & Otter Creek at Camp Creek Road & $7 / 11 / 2010$ & EMAP_R500 & Thienemannimyia gr. & 5 \\
\hline ATG_CCC & Otter Creek at CCC Camp USFS & $7 / 11 / 2010$ & EMAP_R500 & Agabus & 6 \\
\hline ATG_CCC & Otter Creek at CCC Camp USFS & $7 / 11 / 2010$ & EMAP_R500 & Baetis tricaudatus & 13 \\
\hline
\end{tabular}

Appendix B - 15 


\begin{tabular}{|c|c|c|c|c|c|}
\hline StationID & Site_Name & $\begin{array}{l}\text { Start } \\
\text { Date }\end{array}$ & Activity & Taxon & Abund \\
\hline ATG_CCC & Otter Creek at CCC Camp USFS & $7 / 11 / 2010$ & EMAP_R500 & Berosus & 8 \\
\hline ATG_CCC & Otter Creek at CCC Camp USFS & $7 / 11 / 2010$ & EMAP_R500 & Caenis latipennis & 23 \\
\hline ATG_CCC & Otter Creek at CCC Camp USFS & $7 / 11 / 2010$ & EMAP_R500 & Centroptilum & 4 \\
\hline ATG_CCC & Otter Creek at CCC Camp USFS & $7 / 11 / 2010$ & EMAP_R500 & Cheumatopsyche & 33 \\
\hline ATG_CCC & Otter Creek at CCC Camp USFS & $7 / 11 / 2010$ & EMAP_R500 & Chrysops & 2 \\
\hline ATG_CCC & Otter Creek at CCC Camp USFS & $7 / 11 / 2010$ & EMAP_R500 & Cricotopus & 11 \\
\hline ATG_CCC & Otter Creek at CCC Camp USFS & $7 / 11 / 2010$ & EMAP_R500 & Cricotopus bicinctus gr. & 8 \\
\hline ATG_CCC & Otter Creek at CCC Camp USFS & $7 / 11 / 2010$ & EMAP_R500 & Dubiraphia & 3 \\
\hline ATG_CCC & Otter Creek at CCC Camp USFS & $7 / 11 / 2010$ & EMAP_R500 & Enallagma & 6 \\
\hline ATG_CCC & Otter Creek at CCC Camp USFS & $7 / 11 / 2010$ & EMAP_R500 & Erpobdellidae & 2 \\
\hline ATG_CCC & Otter Creek at CCC Camp USFS & $7 / 11 / 2010$ & EMAP_R500 & Eukiefferiella claripennis gr. & 1 \\
\hline ATG_CCC & Otter Creek at CCC Camp USFS & $7 / 11 / 2010$ & EMAP_R500 & Gammarus & 13 \\
\hline ATG_CCC & Otter Creek at CCC Camp USFS & $7 / 11 / 2010$ & EMAP_R500 & Gyraulus parvus & 9 \\
\hline ATG_CCC & Otter Creek at CCC Camp USFS & $7 / 11 / 2010$ & EMAP_R500 & Haliplus & 4 \\
\hline ATG_CCC & Otter Creek at CCC Camp USFS & $7 / 11 / 2010$ & EMAP_R500 & Hesperophylax designatus & 25 \\
\hline ATG_CCC & Otter Creek at CCC Camp USFS & $7 / 11 / 2010$ & EMAP_R500 & Hyalella azteca & 68 \\
\hline ATG_CCC & Otter Creek at CCC Camp USFS & $7 / 11 / 2010$ & EMAP_R500 & Hydrachna & 2 \\
\hline ATG_CCC & Otter Creek at CCC Camp USFS & $7 / 11 / 2010$ & EMAP_R500 & Lestes & 8 \\
\hline ATG_CCC & Otter Creek at CCC Camp USFS & $7 / 11 / 2010$ & EMAP_R500 & Libellula & 2 \\
\hline ATG_CCC & Otter Creek at CCC Camp USFS & $7 / 11 / 2010$ & EMAP_R500 & Limnephilus & 14 \\
\hline ATG_CCC & Otter Creek at CCC Camp USFS & $7 / 11 / 2010$ & EMAP_R500 & Ochthebius & 2 \\
\hline ATG_CCC & Otter Creek at CCC Camp USFS & $7 / 11 / 2010$ & EMAP_R500 & Optioservus & 10 \\
\hline ATG_CCC & Otter Creek at CCC Camp USFS & $7 / 11 / 2010$ & EMAP_R500 & Orconectes virilis & 1 \\
\hline ATG_CCC & Otter Creek at CCC Camp USFS & $7 / 11 / 2010$ & EMAP_R500 & Oreodytes & 2 \\
\hline ATG_CCC & Otter Creek at CCC Camp USFS & $7 / 11 / 2010$ & EMAP_R500 & Orthocladius & 22 \\
\hline ATG_CCC & Otter Creek at CCC Camp USFS & $7 / 11 / 2010$ & EMAP_R500 & Physella gyrina & 154 \\
\hline ATG_CCC & Otter Creek at CCC Camp USFS & $7 / 11 / 2010$ & EMAP_R500 & Probezzia & 2 \\
\hline ATG_CCC & Otter Creek at CCC Camp USFS & $7 / 11 / 2010$ & EMAP_R500 & Procladius & 8 \\
\hline ATG_CCC & Otter Creek at CCC Camp USFS & $7 / 11 / 2010$ & EMAP_R500 & Prodiamesa & 1 \\
\hline ATG_CCC & Otter Creek at CCC Camp USFS & $7 / 11 / 2010$ & EMAP_R500 & Simulium & 143 \\
\hline ATG_CCC & Otter Creek at CCC Camp USFS & 7/11/2010 & EMAP_R500 & Stagnicola & 7 \\
\hline ATG_CCC & Otter Creek at CCC Camp USFS & $7 / 11 / 2010$ & EMAP_R500 & Tubificidae & 14 \\
\hline ATG_235 & South Fork Taylor Creek & $7 / 11 / 2010$ & EMAP_R500 & Aeshna palmata & 2 \\
\hline ATG_235 & South Fork Taylor Creek & $7 / 11 / 2010$ & EMAP_R500 & Berosus & 14 \\
\hline
\end{tabular}




\begin{tabular}{|c|c|c|c|c|c|}
\hline StationID & Site_Name & $\begin{array}{l}\text { Start } \\
\text { Date }\end{array}$ & Activity & Taxon & Abund \\
\hline ATG_235 & South Fork Taylor Creek & 7/11/2010 & EMAP_R500 & Buenoa & 12 \\
\hline ATG_235 & South Fork Taylor Creek & $7 / 11 / 2010$ & EMAP_R500 & Caenis latipennis & 44 \\
\hline ATG_235 & South Fork Taylor Creek & 7/11/2010 & EMAP_R500 & Callibaetis & 98 \\
\hline ATG_235 & South Fork Taylor Creek & 7/11/2010 & EMAP_R500 & Chironominae & 43 \\
\hline ATG_235 & South Fork Taylor Creek & $7 / 11 / 2010$ & EMAP_R500 & Corixidae & 52 \\
\hline ATG_235 & South Fork Taylor Creek & 7/11/2010 & EMAP_R500 & Culicoides & 2 \\
\hline ATG_235 & South Fork Taylor Creek & $7 / 11 / 2010$ & EMAP_R500 & Dolichopodidae & 1 \\
\hline ATG_235 & South Fork Taylor Creek & $7 / 11 / 2010$ & EMAP_R500 & Enallagma & 16 \\
\hline ATG_235 & South Fork Taylor Creek & 7/11/2010 & EMAP_R500 & Haliplus & 1 \\
\hline ATG_235 & South Fork Taylor Creek & 7/11/2010 & EMAP_R500 & Helophorus & 1 \\
\hline ATG_235 & South Fork Taylor Creek & $7 / 11 / 2010$ & EMAP_R500 & Hyalella azteca & 66 \\
\hline ATG_235 & South Fork Taylor Creek & 7/11/2010 & EMAP_R500 & Hydrobius & 1 \\
\hline ATG_235 & South Fork Taylor Creek & $7 / 11 / 2010$ & EMAP_R500 & Illybius & 6 \\
\hline ATG_235 & South Fork Taylor Creek & $7 / 11 / 2010$ & EMAP_R500 & Laccophilus & 1 \\
\hline ATG_235 & South Fork Taylor Creek & $7 / 11 / 2010$ & EMAP_R500 & Lestes & 21 \\
\hline ATG_235 & South Fork Taylor Creek & 7/11/2010 & EMAP_R500 & Libellula & 2 \\
\hline ATG_235 & South Fork Taylor Creek & 7/11/2010 & EMAP_R500 & Orthocladiinae & 13 \\
\hline ATG_235 & South Fork Taylor Creek & 7/11/2010 & EMAP_R500 & Physella acuta & 7 \\
\hline ATG_235 & South Fork Taylor Creek & $7 / 11 / 2010$ & EMAP_R500 & Physella gyrina & 18 \\
\hline ATG_235 & South Fork Taylor Creek & $7 / 11 / 2010$ & EMAP_R500 & Procladius & 2 \\
\hline ATG_235 & South Fork Taylor Creek & $7 / 11 / 2010$ & EMAP_R500 & Simulium & 8 \\
\hline ATG_235 & South Fork Taylor Creek & $7 / 11 / 2010$ & EMAP_R500 & Stagnicola caperata & 13 \\
\hline ATG_235 & South Fork Taylor Creek & $7 / 11 / 2010$ & EMAP_R500 & Sympetrum & 6 \\
\hline ATG_235 & South Fork Taylor Creek & $7 / 11 / 2010$ & EMAP_R500 & Tabanus & 1 \\
\hline ATG_235 & South Fork Taylor Creek & $7 / 11 / 2010$ & EMAP_R500 & Tanypodinae & 54 \\
\hline
\end{tabular}





\section{Appendix C. Raw Fish Data and IBI Metric Calculations Collected from Upper Hanging Woman and Otter Creek Sites}





\begin{tabular}{lc}
\multicolumn{1}{c}{ ATG_124 } & \\
\cline { 1 - 2 } Species & Number \\
Brassy Minnow & 15 \\
Common Carp* & 0 \\
Fathead Minnow & 2 \\
Green Sunfish* & 0 \\
Lake Chub & 0 \\
Pumpkinseed* & 0 \\
White Sucker & 0 \\
\hline
\end{tabular}

\begin{tabular}{|c|c|c|c|}
\hline Metrics & Counts & $\begin{array}{c}\text { Adjusted } \\
\text { Value }\end{array}$ & Score \\
\hline Number of Native Fish Species to MT & 2 & 11.464 & 64 \\
\hline Number of Native Fish Families to MT & 1 & 2.750 & 51 \\
\hline Number of non-tolerant Cyprinid species & 1 & 6.536 & 93 \\
\hline Number of Sucker and Catfish Species & 0 & 5.791 & 64 \\
\hline Number of Moderately Intolerant Species & 0 & 7.459 & 82 \\
\hline Proportion of Tolerant individuals & 11.765 & none & 88 \\
\hline $\begin{array}{l}\text { Proportion out of the Total Number of Fish } \\
\text { That Were Insect-Eating Minnows }\end{array}$ & 0 & none & 0 \\
\hline $\begin{array}{l}\text { Total Number of Fish Species That Prefer } \\
\text { to Eat Insects That Live on the Stream } \\
\text { Bottom }\end{array}$ & 0 & 4.471 & 76 \\
\hline $\begin{array}{l}\text { Proportion of the Total Number of Fish } \\
\text { That Require Rocks to Lay Eggs }\end{array}$ & 0 & none & 0 \\
\hline $\begin{array}{l}\text { Proportion of the Total Number of } \\
\text { Individual Fish That Do Not Require } \\
\text { Rocks to Lay Eggs, But Have Parental } \\
\text { Care of Eggs }\end{array}$ & 11.765 & none & 87 \\
\hline $\begin{array}{l}\text { Proportion of the Total Number of Fish } \\
\text { Sampled That Were Native to Montana }\end{array}$ & 100 & none & 100 \\
\hline \multicolumn{4}{|l|}{$\begin{array}{l}\text { Number of Fish Species Collected That } \\
\text { Were Long-Lived }\end{array}$} \\
\hline Totals & & & 704 \\
\hline Final Calculation & & & $704 / 1100$ \\
\hline IBI Score & & & $64 \%$ \\
\hline
\end{tabular}




\begin{tabular}{lc}
\hline \multicolumn{1}{c}{ ATG_141 } & \\
\cline { 1 - 2 } Species & Number \\
Brassy Minnow & 10 \\
Common Carp* & 0 \\
Fathead Minnow & 16 \\
Green Sunfish* & 16 \\
Lake Chub & 0 \\
Pumpkinseed* & 0 \\
White Sucker & 0 \\
\hline
\end{tabular}

\begin{tabular}{|c|c|c|c|}
\hline Metrics & Counts & $\begin{array}{l}\text { Adjusted } \\
\text { Value }\end{array}$ & Score \\
\hline Number of Native Fish Species to Montana & 2 & 10.252 & 57 \\
\hline Number of Native Fish Families to MT & 1 & 2.530 & 47 \\
\hline Number of non-tolerant Cyprinid species & 1 & 7.003 & 100 \\
\hline Number of Sucker and Catfish Species & 0 & 5.063 & 56 \\
\hline Number of Moderately Intolerant Species & 0 & 6.522 & 72 \\
\hline Proportion of Tolerant individuals & 76.190 & none & 19 \\
\hline $\begin{array}{l}\text { Proportion out of the Total Number of Fish } \\
\text { That Were Insect-Eating Minnows }\end{array}$ & 0 & none & 0 \\
\hline $\begin{array}{l}\text { Total Number of Fish Species That Prefer to } \\
\text { Eat Insects That Live on the Stream Bottom }\end{array}$ & 0 & 3.909 & 66 \\
\hline $\begin{array}{l}\text { Proportion of the Total Number of Fish That } \\
\text { Require Rocks to Lay Eggs }\end{array}$ & 0 & none & 0 \\
\hline $\begin{array}{l}\text { Proportion of the Total Number of Individual } \\
\text { Fish That Do Not Require Rocks to Lay } \\
\text { Eggs, But Have Parental Care of Eggs }\end{array}$ & 38.095 & none & 57 \\
\hline $\begin{array}{l}\text { Proportion of the Total Number of Fish } \\
\text { Sampled That Were Native to Montana }\end{array}$ & 61.905 & none & 62 \\
\hline \multicolumn{4}{|l|}{$\begin{array}{l}\text { Number of Fish Species Collected That Were } \\
\text { Long-Lived }\end{array}$} \\
\hline Totals & & & 536 \\
\hline Final Calculation & & & $536 / 1100$ \\
\hline IBI Score & & & $49 \%$ \\
\hline
\end{tabular}




\begin{tabular}{lc}
\hline \multicolumn{1}{c}{ ATG_145 } & \\
\cline { 1 - 2 } Species & Number \\
Brassy Minnow & 0 \\
Common Carp* & 0 \\
Fathead Minnow & 50 \\
Green Sunfish* & 0 \\
Lake Chub & 0 \\
Pumpkinseed* & 0 \\
White Sucker & 0 \\
\hline
\end{tabular}

\begin{tabular}{|c|c|c|c|c|}
\hline Metrics & Counts & $\begin{array}{l}\text { Adjusted } \\
\text { Value }\end{array}$ & Score & Best \\
\hline Number of Native Fish Species to MT & 1 & 8.540 & 47 & 100 \\
\hline Number of Native Fish Families to MT & 1 & 2.401 & 44 & 100 \\
\hline Number of non-tolerant Cyprinid species & 0 & 8.276 & 118 & 100 \\
\hline Number of Sucker and Catfish Species & 0 & 4.636 & 51 & 100 \\
\hline Number of Moderately Intolerant Species & 0 & 5.971 & 66 & 100 \\
\hline Proportion of Tolerant individuals & 100 & none & -6 & 100 \\
\hline $\begin{array}{l}\text { Proportion out of the Total Number of Fish } \\
\text { That Were Insect-Eating Minnows }\end{array}$ & 0 & none & 0 & 100 \\
\hline $\begin{array}{l}\text { Total Number of Fish Species That Prefer to } \\
\text { Eat Insects That Live on the Stream Bottom }\end{array}$ & 0 & 3.579 & 61 & 100 \\
\hline $\begin{array}{l}\text { Proportion of the Total Number of Fish That } \\
\text { Require Rocks to Lay Eggs }\end{array}$ & 0 & none & 0 & 100 \\
\hline $\begin{array}{l}\text { Proportion of the Total Number of Individual } \\
\text { Fish That Do Not Require Rocks to Lay Eggs, } \\
\text { But Have Parental Care of Eggs }\end{array}$ & 100 & none & -14 & 100 \\
\hline $\begin{array}{l}\text { Proportion of the Total Number of Fish } \\
\text { Sampled That Were Native to Montana }\end{array}$ & 100 & none & 100 & 100 \\
\hline $\begin{array}{l}\text { Number of Fish Species Collected That Were } \\
\text { Long-Lived }\end{array}$ & & & & \\
\hline Totals & \multirow{2}{*}{\multicolumn{4}{|c|}{$673 / 1000$}} \\
\hline Final Calculation & & & & \\
\hline IBI Score & \multicolumn{4}{|c|}{$43 \%$} \\
\hline
\end{tabular}




\begin{tabular}{lcc}
\multicolumn{1}{c}{ ATG_150+ATG_151 } & \multicolumn{2}{c}{ Number } \\
\cline { 1 - 2 } Species & 0 & 0 \\
Brassy Minnow & 0 & 0 \\
Common Carp* & 40 & 20 \\
Fathead Minnow & 0 & 0 \\
Green Sunfish* & 0 & 0 \\
Lake Chub & 0 & 0 \\
Pumpkinseed* & 0 & 0 \\
White Sucker & 40 & 20
\end{tabular}

\begin{tabular}{|c|c|c|c|c|}
\hline Metrics & Counts & $\begin{array}{c}\text { Adjusted } \\
\text { Value }\end{array}$ & Score & Best \\
\hline Number of Native Fish Species to Montana & 1 & 8.540 & 47 & 100 \\
\hline Number of Native Fish Families to MT & 1 & 2.401 & 44 & 100 \\
\hline Number of non-tolerant Cyprinid species & 0 & 8.276 & 118 & 100 \\
\hline Number of Sucker and Catfish Species & 0 & 4.636 & 51 & 100 \\
\hline Number of Moderately Intolerant Species & 0 & 5.971 & 66 & 100 \\
\hline Proportion of Tolerant individuals & 100 & none & -6 & 100 \\
\hline $\begin{array}{l}\text { Proportion out of the Total Number of Fish } \\
\text { That Were Insect-Eating Minnows }\end{array}$ & 0 & none & 0 & 100 \\
\hline $\begin{array}{l}\text { Total Number of Fish Species That Prefer to } \\
\text { Eat Insects That Live on the Stream Bottom }\end{array}$ & 0 & 3.579 & 61 & 100 \\
\hline $\begin{array}{l}\text { Proportion of the Total Number of Fish That } \\
\text { Require Rocks to Lay Eggs }\end{array}$ & 0 & none & 0 & 100 \\
\hline $\begin{array}{l}\text { Proportion of the Total Number of Individual } \\
\text { Fish That Do Not Require Rocks to Lay Eggs, } \\
\text { But Have Parental Care of Eggs }\end{array}$ & 100 & none & -14 & 100 \\
\hline $\begin{array}{l}\text { Proportion of the Total Number of Fish } \\
\text { Sampled That Were Native to Montana }\end{array}$ & 100 & none & 100 & 100 \\
\hline $\begin{array}{l}\text { Number of Fish Species Collected That Were } \\
\text { Long-Lived }\end{array}$ & & & & \\
\hline Totals & & & 468 & 1100 \\
\hline Final Calculation & \multicolumn{4}{|c|}{$468 / 1100$} \\
\hline IBI Score & \multicolumn{4}{|c|}{$43 \%$} \\
\hline
\end{tabular}




\begin{tabular}{lc}
\hline \multicolumn{1}{c}{ ATG_196 } & \\
\cline { 1 - 2 } Species & Number \\
Brassy Minnow & 0 \\
Common Carp* & 2 \\
Fathead Minnow & 482 \\
Green Sunfish* & 2 \\
Lake Chub & 0 \\
Pumpkinseed* & 0 \\
White Sucker & 0 \\
\hline
\end{tabular}

\begin{tabular}{|c|c|c|c|c|}
\hline Metrics & Counts & $\begin{array}{l}\text { Adjusted } \\
\text { Value } \\
\end{array}$ & Score & Best \\
\hline Number of Native Fish Species to Montana & 1 & 8.540 & 47 & 100 \\
\hline Number of Native Fish Families to MT & 1 & 2.401 & 44 & 100 \\
\hline Number of non-tolerant Cyprinid species & 0 & 8.276 & 118 & 100 \\
\hline Number of Sucker and Catfish Species & 0 & 4.636 & 51 & 100 \\
\hline Number of Moderately Intolerant Species & 0 & 5.971 & 66 & 100 \\
\hline Proportion of Tolerant individuals & 100 & none & -6 & 100 \\
\hline $\begin{array}{l}\text { Proportion out of the Total Number of Fish } \\
\text { That Were Insect-Eating Minnows }\end{array}$ & 0 & none & 0 & 100 \\
\hline $\begin{array}{l}\text { Total Number of Fish Species That Prefer to } \\
\text { Eat Insects That Live on the Stream Bottom }\end{array}$ & 0 & 3.579 & 61 & 100 \\
\hline $\begin{array}{l}\text { Proportion of the Total Number of Fish That } \\
\text { Require Rocks to Lay Eggs }\end{array}$ & 0 & none & 0 & 100 \\
\hline $\begin{array}{l}\text { Proportion of the Total Number of Individual } \\
\text { Fish That Do Not Require Rocks to Lay Eggs, } \\
\text { But Have Parental Care of Eggs }\end{array}$ & 100 & none & -14 & 100 \\
\hline $\begin{array}{l}\text { Proportion of the Total Number of Fish } \\
\text { Sampled That Were Native to Montana } \\
\text { Number of Fish Species Collected That Were } \\
\text { Long-Lived }\end{array}$ & 99.177 & none & 99 & 100 \\
\hline Totals & & & 467 & 1100 \\
\hline Final Calculation & & & $467 / 1000$ & \\
\hline IBI Score & & & $42 \%$ & \\
\hline
\end{tabular}




\begin{tabular}{lc}
\hline \multicolumn{1}{c}{ ATG_200 } & \\
\cline { 1 - 2 } Species & Number \\
Brassy Minnow & 15 \\
Common Carp* & 0 \\
Fathead Minnow & 55 \\
Green Sunfish* & 0 \\
Lake Chub & 0 \\
Pumpkinseed* & 0 \\
White Sucker & 5 \\
\hline
\end{tabular}

\begin{tabular}{|c|c|c|c|c|}
\hline Metrics & Counts & $\begin{array}{l}\text { Adjusted } \\
\text { Value }\end{array}$ & Score & Best \\
\hline Number of Native Fish Species to MT & 3 & 11.782 & 65 & 100 \\
\hline Number of Native Fish Families to MT & 2 & 3.626 & 67 & 100 \\
\hline Number of non-tolerant Cyprinid species & 1 & 6.799 & 97 & 100 \\
\hline Number of Sucker and Catfish Species & 1 & 6.381 & 70 & 100 \\
\hline Number of Moderately Intolerant Species & 0 & 6.931 & 76 & 100 \\
\hline Proportion of Tolerant individuals & 80 & none & 15 & 100 \\
\hline $\begin{array}{l}\text { Proportion out of the Total Number of Fish } \\
\text { That Were Insect-Eating Minnows }\end{array}$ & 0 & none & 0 & 100 \\
\hline $\begin{array}{l}\text { Total Number of Fish Species That Prefer to } \\
\text { Eat Insects That Live on the Stream Bottom }\end{array}$ & 0 & 4.154 & 71 & 100 \\
\hline $\begin{array}{l}\text { Proportion of the Total Number of Fish That } \\
\text { Require Rocks to Lay Eggs }\end{array}$ & 0 & none & 0 & 100 \\
\hline $\begin{array}{l}\text { Proportion of the Total Number of Individual } \\
\text { Fish That Do Not Require Rocks to Lay Eggs, } \\
\text { But Have Parental Care of Eggs }\end{array}$ & 73.333 & none & 17 & 100 \\
\hline $\begin{array}{l}\text { Proportion of the Total Number of Fish } \\
\text { Sampled That Were Native to Montana } \\
\text { Number of Fish Species Collected That Were } \\
\text { Long-Lived }\end{array}$ & 100 & none & 100 & 100 \\
\hline Totals & & & 579 & 1100 \\
\hline Final Calculation & & & $579 / 1100$ & \\
\hline IBI Score & & & $53 \%$ & \\
\hline
\end{tabular}




\begin{tabular}{lc}
\hline \multicolumn{1}{c}{ ATG_201 } & \\
\cline { 1 - 2 } Species & Number \\
Brassy Minnow & 72 \\
Common Carp* & 0 \\
Fathead Minnow & 35 \\
Green Sunfish* & 0 \\
Lake Chub & 10 \\
Pumpkinseed* & 0 \\
White Sucker & 0 \\
\hline
\end{tabular}

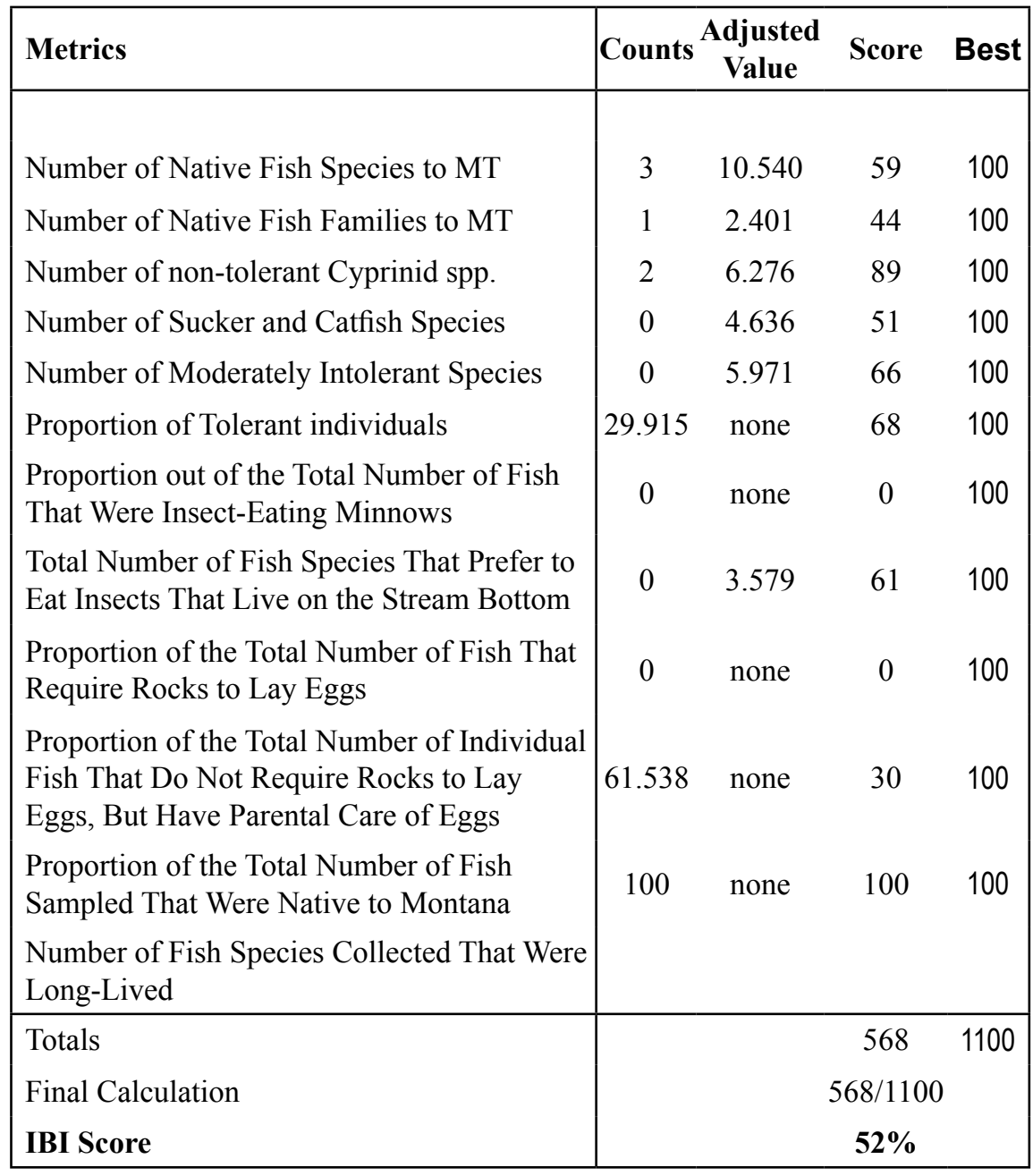




\begin{tabular}{lc}
\hline \multicolumn{1}{c}{ ATG_202 } & \\
\cline { 1 - 2 } Species & Number \\
Brassy Minnow & 40 \\
Common Carp* & 0 \\
Fathead Minnow & 5 \\
Green Sunfish* & 0 \\
Lake Chub & 55 \\
Pumpkinseed* & 0 \\
White Sucker & 25 \\
\hline
\end{tabular}

\begin{tabular}{|c|c|c|c|c|}
\hline Metrics & Counts & $\begin{array}{l}\text { Adjusted } \\
\text { Value }\end{array}$ & Score & Best \\
\hline Number of Native Fish Species to MT & 4 & 11.540 & 64 & 100 \\
\hline Number of Native Fish Families to MT & 2 & 3.401 & 63 & 100 \\
\hline Number of non-tolerant Cyprinid species & 1 & 7.276 & 104 & 100 \\
\hline Number of Sucker and Catfish Species & 1 & 5.636 & 62 & 100 \\
\hline Number of Moderately Intolerant Species & 0 & 5.971 & 66 & 100 \\
\hline Proportion of Tolerant individuals & 24 & none & 75 & 100 \\
\hline $\begin{array}{l}\text { Proportion out of the Total Number of Fish } \\
\text { That Were Insect-Eating Minnows }\end{array}$ & 0 & none & 0 & 100 \\
\hline $\begin{array}{l}\text { Total Number of Fish Species That Prefer to } \\
\text { Eat Insects That Live on the Stream Bottom }\end{array}$ & 1 & 4.579 & 78 & 100 \\
\hline $\begin{array}{l}\text { Proportion of the Total Number of Fish That } \\
\text { Require Rocks to Lay Eggs }\end{array}$ & 20 & none & 24 & 100 \\
\hline $\begin{array}{l}\text { Proportion of the Total Number of Individual } \\
\text { Fish That Do Not Require Rocks to Lay Eggs, } \\
\text { But Have Parental Care of Eggs }\end{array}$ & 67.290 & none & 23 & 100 \\
\hline $\begin{array}{l}\text { Proportion of the Total Number of Fish } \\
\text { Sampled That Were Native to Montana }\end{array}$ & 100 & none & 100 & 100 \\
\hline $\begin{array}{l}\text { Number of Fish Species Collected That Were } \\
\text { Long-Lived }\end{array}$ & & & & \\
\hline Totals & & & 659 & 1100 \\
\hline Final Calculation & \multicolumn{4}{|c|}{$659 / 1100$} \\
\hline IBI Score & \multicolumn{4}{|c|}{$60 \%$} \\
\hline
\end{tabular}




\begin{tabular}{lc}
\hline \multicolumn{1}{c}{ ATG_203 } & \\
\cline { 1 - 2 } Species & Number \\
Brassy Minnow & 22 \\
Common Carp* & 0 \\
Fathead Minnow & 110 \\
Green Sunfish* & 0 \\
Lake Chub & 0 \\
Pumpkinseed* & 0 \\
White Sucker & 5 \\
\hline
\end{tabular}

\begin{tabular}{|c|c|c|c|c|}
\hline Metrics & Counts & $\begin{array}{l}\text { Adjusted } \\
\text { Value }\end{array}$ & Score & Best \\
\hline Number of Native Fish Species to MT & 3 & 10.540 & 59 & 100 \\
\hline Number of Native Fish Families to MT & 2 & 3.401 & 63 & 100 \\
\hline Number of non-tolerant Cyprinid species & 1 & 7.276 & 104 & 100 \\
\hline Number of Sucker and Catfish Species & 1 & 5.636 & 62 & 100 \\
\hline Number of Moderately Intolerant Species & 0 & 5.971 & 66 & 100 \\
\hline Proportion of Tolerant individuals & 83.942 & none & 11 & 100 \\
\hline $\begin{array}{l}\text { Proportion out of the Total Number of Fish } \\
\text { That Were Insect-Eating Minnows }\end{array}$ & 0 & none & 0 & 100 \\
\hline $\begin{array}{l}\text { Total Number of Fish Species That Prefer to } \\
\text { Eat Insects That Live on the Stream Bottom }\end{array}$ & 1 & 4.579 & 78 & 100 \\
\hline $\begin{array}{l}\text { Proportion of the Total Number of Fish That } \\
\text { Require Rocks to Lay Eggs }\end{array}$ & 3.650 & none & 4 & 100 \\
\hline $\begin{array}{l}\text { Proportion of the Total Number of Individual } \\
\text { Fish That Do Not Require Rocks to Lay } \\
\text { Eggs, But Have Parental Care of Eggs }\end{array}$ & 80.292 & none & 9 & 100 \\
\hline $\begin{array}{l}\text { Proportion of the Total Number of Fish } \\
\text { Sampled That Were Native to Montana }\end{array}$ & 100 & none & 100 & 100 \\
\hline $\begin{array}{l}\text { Number of Fish Species Collected That Were } \\
\text { Long-Lived }\end{array}$ & & & & \\
\hline Totals & & & 555 & 1100 \\
\hline Final Calculation & \multicolumn{4}{|c|}{$555 / 1100$} \\
\hline IBI Score & \multicolumn{4}{|c|}{$50 \%$} \\
\hline
\end{tabular}




\begin{tabular}{lc}
\hline \multicolumn{1}{c}{ ATG_235 } & \\
\cline { 1 - 2 } Species & Number \\
Brassy Minnow & 0 \\
Common Carp* & 0 \\
Fathead Minnow & 0 \\
Green Sunfish* & 0 \\
Lake Chub & 25 \\
Pumpkinseed* & 0 \\
White Sucker & 0 \\
\hline
\end{tabular}

\begin{tabular}{|c|c|c|c|c|}
\hline Metrics & Counts & $\begin{array}{l}\text { Adjusted } \\
\text { Value }\end{array}$ & Score & Best \\
\hline Number of Native Fish Species to MT & 1 & 14.186 & 79 & 100 \\
\hline Number of Native Fish Families to MT & 1 & 3.425 & 63 & 100 \\
\hline Number of non-tolerant Cyprinid species & 1 & 5.104 & 73 & 100 \\
\hline Number of Sucker and Catfish Species & 0 & 8.025 & 88 & 100 \\
\hline Number of Moderately Intolerant Species & 0 & 10.336 & 114 & 100 \\
\hline Proportion of Tolerant individuals & 0 & none & 100 & 100 \\
\hline $\begin{array}{l}\text { Proportion out of the Total Number of Fish } \\
\text { That Were Insect-Eating Minnows }\end{array}$ & 0 & none & 0 & 100 \\
\hline $\begin{array}{l}\text { Total Number of Fish Species That Prefer to } \\
\text { Eat Insects That Live on the Stream Bottom }\end{array}$ & 0 & 3.664 & 62 & 100 \\
\hline $\begin{array}{l}\text { Proportion of the Total Number of Fish That } \\
\text { Require Rocks to Lay Eggs }\end{array}$ & 0 & none & 0 & 100 \\
\hline $\begin{array}{l}\text { Proportion of the Total Number of Individual } \\
\text { Fish That Do Not Require Rocks to Lay Eggs, } \\
\text { But Have Parental Care of Eggs }\end{array}$ & 0 & none & 100 & 100 \\
\hline $\begin{array}{l}\text { Proportion of the Total Number of Fish } \\
\text { Sampled That Were Native to Montana }\end{array}$ & 100 & none & 100 & 100 \\
\hline $\begin{array}{l}\text { Number of Fish Species Collected That Were } \\
\text { Long-Lived }\end{array}$ & & & & \\
\hline Totals & & & 779 & 1100 \\
\hline Final Calculation & \multicolumn{4}{|c|}{$779 / 1100$} \\
\hline IBI Score & \multicolumn{4}{|c|}{$71 \%$} \\
\hline
\end{tabular}




\begin{tabular}{lc}
\hline \multicolumn{1}{c}{ ATG_CCC } & \\
\cline { 1 - 2 } Species & Number \\
Brassy Minnow & 61 \\
Common Carp* & 0 \\
Fathead Minnow & 15 \\
Green Sunfish* & 0 \\
Lake Chub & 10 \\
Pumpkinseed* & 4 \\
White Sucker & 5 \\
\hline
\end{tabular}

\begin{tabular}{|c|c|c|c|c|}
\hline Metrics & Counts & $\begin{array}{l}\text { Adjusted } \\
\text { Value }\end{array}$ & Score & Best \\
\hline Number of Native Fish Species to MT & 4.00 & 9.493 & 53 & 100 \\
\hline Number of Native Fish Families to MT & 2.00 & 3.029 & 56 & 100 \\
\hline Number of non-tolerant Cyprinid species & 2.00 & 7.064 & 101 & 100 \\
\hline Number of Sucker and Catfish Species & 1.00 & 4.407 & 49 & 100 \\
\hline Number of Moderately Intolerant Species & 0.00 & 4.388 & 48 & 100 \\
\hline Proportion of Tolerant individuals & 21.05 & none & 78 & 100 \\
\hline $\begin{array}{l}\text { Proportion out of the Total Number of Fish } \\
\text { That Were Insect-Eating Minnows }\end{array}$ & 0.00 & none & 0 & 100 \\
\hline $\begin{array}{l}\text { Total Number of Fish Species That Prefer to } \\
\text { Eat Insects That Live on the Stream Bottom }\end{array}$ & 1.00 & 3.630 & 62 & 100 \\
\hline $\begin{array}{l}\text { Proportion of the Total Number of Fish That } \\
\text { Require Rocks to Lay Eggs }\end{array}$ & 5.21 & none & 6 & 100 \\
\hline $\begin{array}{l}\text { Proportion of the Total Number of Individual } \\
\text { Fish That Do Not Require Rocks to Lay Eggs, } \\
\text { But Have Parental Care of Eggs }\end{array}$ & 15.79 & none & 82 & 100 \\
\hline $\begin{array}{l}\text { Proportion of the Total Number of Fish } \\
\text { Sampled That Were Native to Montana }\end{array}$ & 100.00 & none & 100 & 100 \\
\hline $\begin{array}{l}\text { Number of Fish Species Collected That Were } \\
\text { Long-Lived }\end{array}$ & & & & \\
\hline Totals & & & 634 & 1100 \\
\hline Final Calculation & \multicolumn{4}{|c|}{$634 / 1100$} \\
\hline IBI Score & \multicolumn{4}{|c|}{$\mathbf{5 8 \%}$} \\
\hline
\end{tabular}

